\title{
On High Moments of Strongly Diluted Large Wigner Random Matrices *i
}

\author{
O. Khorunzhiy \\ Université de Versailles - Saint-Quentin \\ 45, Avenue des Etats-Unis, 78035 Versailles, FRANCE \\ e-mail: oleksiy.khorunzhiy@uvsq.fr
}

July 3, 2018

\begin{abstract}
We consider a dilute version of the Wigner ensemble of $n \times n$ random real symmetric matrices $H^{(n, \rho)}$, where $\rho$ denotes the average number of non-zero elements per row. We study the asymptotic properties of the moments $M_{2 s}^{(n, \rho)}=\mathbf{E} \operatorname{Tr}\left(H^{(n, \rho)}\right)^{2 s}$ in the limit when $n, s$ and $\rho$ tend to infinity.

Our main result is that the sequence $M_{2 s_{n}}^{\left(n, \rho_{n}\right)}$ with $s_{n}=\left\lfloor\chi \rho_{n}\right\rfloor, \chi>0, \rho_{n} \rightarrow \infty$ and $\rho_{n}=o\left(n^{1 / 5}\right)$ is asymptotically close to a sequence of numbers $n \hat{m}_{s_{n}}^{\left(\rho_{n}\right)}$, where $\left\{\hat{m}_{s}^{(\rho)}\right\}_{s \geq 0}$ are determined by an explicit recurrence that involves the second and the fourth moments of the random variables $\left(H^{(n, \rho)}\right)_{i j}, V_{2}$ and $V_{4}$, respectively. This recurrent relation generalizes the one that determines the moments of the Wigner's semicircle law given by $m_{s}=\lim _{\rho \rightarrow \infty} \hat{m}_{s}(\rho), s \in \mathbb{N}$. It shows that the spectral properties of random matrices at the edge of the limiting spectrum regarded in the asymptotic regime of the strong dilution essentially differ from those observed in the cases of moderate and weak dilution, where the dependence on the fourth moment $V_{4}$ does not intervene.
\end{abstract}

Running title: Strongly Diluted Wigner Random Matrices

\section{Introduction, main results and discussion}

Spectral theory of high dimensional random matrices represents an intensively developing branch of modern mathematical physics that reveals deep links between probability theory, analysis, combinatorics and other various fields of mathematics (see monographs $[1,18]$ ). The first studies of spectral properties of random matrices of infinitely increasing dimensions were started by E. Wigner (see e. g. [28]), where the ensemble of real symmetric matrices of the form

$$
\left(A^{(n)}\right)_{i j}=\frac{1}{\sqrt{n}} a_{i j}
$$

\footnotetext{
*Key words: random matrices, Wigner ensemble, dilute random matrices

${ }^{\dagger}$ MSC: $15 \mathrm{~B} 52$
} 
was introduced and the limiting eigenvalue distribution of $A^{(n)}, n \rightarrow \infty$ was determined explicitly. The random matrix entries of $A^{(n)}(1.1)$ are given by jointly independent random variables $\left\{a_{i j}, i \leq j\right\}$ that have all moments finite and the odd moments zero. At present, this ensemble is referred as the Wigner ensemble of random matrices. It was proved in [28] that the eigenvalue distribution of $A^{(n)}$ converges in average as $n \rightarrow \infty$ to a non-random limit with the density of the semi-circle form. At present this convergence is widely known as the semicircle (or Wigner) law for random matrix ensembles.

The semicircle law was generalized in several directions. One group of generalizations concerns the properties of the probability distributions of elements $a_{i j}$, another one is related with the studies of the spectral norm of $A^{(n)}$ and other local properties of the eigenvalue distribution at the border of the limiting spectrum or inside of it.

A large number of works is related with various generalizations of the Wigner ensemble that involve modifications of the random matrix entries. In the present paper we study one of such generalizations given by the ensemble of dilute random matrices. We consider a family of real symmetric random matrices $\left\{H^{(n, \rho)}\right\}$ whose elements are determined by equality

$$
\left(H^{(n, \rho)}\right)_{i j}=a_{i j} b_{i j}^{(n, \rho)}, \quad 1 \leq i \leq j \leq n,
$$

where $\mathfrak{A}=\left\{a_{i j}, 1 \leq i \leq j\right\}$ is an infinite family of jointly independent identically distributed random variables and $\mathfrak{B}_{n}=\left\{b_{i j}^{(n, \rho)}, 1 \leq i \leq j \leq n\right\}$ is a family of jointly independent between themselves random variables that are also independent from $\mathfrak{A}$. We denote by $\mathbf{E}=\mathbf{E}_{n}$ the mathematical expectation with respect to the measure $\mathbf{P}=\mathbf{P}_{n}$ generated by random variables $\left\{\mathfrak{A}, \mathfrak{B}_{n}\right\}$. We assume that the probability distribution of random variables $a_{i j}$ is symmetric and denote their even moments by

$$
V_{2 l}=\mathbf{E}\left(a_{i j}\right)^{2 l}, \quad l=1,2,3, \ldots
$$

Random variables $b_{i j}^{(n, \rho)}$ are proportional to the Bernoulli ones,

$$
b_{i j}^{(n, \rho)}=\frac{1}{\sqrt{\rho}} \begin{cases}1-\delta_{i j}, & \text { with probability } \rho / n, \\ 0, & \text { with probability } 1-\rho / n,\end{cases}
$$

where $\delta_{i j}=\delta_{i, j}$ is the Kronecker $\delta$-symbol. In the case when the dilution parameter $\rho$ is equal to $n$, one gets the Wigner ensemble of real symmetric random matrices $A_{n}$ (1.1). Let us note that the random matrix $B^{(n, \rho)}$ with the entries $\sqrt{\rho} b_{i j}$ (1.3) can be regarded as the adjacency matrix of the Erdős-Rényi random graph [4]. In this interpretation, the dilution parameter $\rho$ represents the average degree of a given vertex of the graph.

The initial interest in the dilute versions of Wigner ensemble was motivated by theoretical physics studies (see for instance, the pioneering works [19], [21] and the review [13] for more references), where the spectral properties of large systems with a number of broken interactions were considered. This kind of random matrices is also important in the studies of various mathematical models, such as random graphs $[5,7,17]$ and many others.

In the present paper we study the asymptotic behavior of the moments of $H^{(n, \rho)}$ given by expression

$$
M_{2 s}^{(n, \rho)}=\mathbf{E}\left(\sum_{i=1}^{n}\left(H^{(n, \rho)}\right)_{i i}^{2 s}\right)=\mathbf{E}\left(\operatorname{Tr}\left(H^{(n, \rho)}\right)^{2 s}\right) .
$$


The moment method represents an effective tool of the spectral theory. It is used in the studies of the spectral properties of large random matrices since the pioneering works of E. Wigner [28]. In particular, the semicircle law was proved initially by the convergence of the moments $M_{2 s}^{(n, n)}$ in the limit of infinite $n$ and given $s$. The principal idea of the Wigner's approach is to consider the trace of the product of random matrices as the sum over the family of trajectories of $2 s$ steps and then to compute the weights of these trajectories given by the mathematical expectation of the products of corresponding random variables.

The moments $M_{2 s}^{(n, n)}$ of Wigner random matrices $A^{(n)}(1.1)$ in the limit $n \rightarrow \infty$ with infinitely increasing $s=s_{n}$ were studied in a long series of papers, where the eigenvalue distribution at the edge of the limiting spectra was studied in more and more details $[2,7,8]$. The crucial step has been performed in papers [22, 23], where the original Wigner's moment method has got powerful and deep improvement. In these studies, the Tracy-Widom law for random matrices $A^{(n)}$ established in the case of normally distributed entries $a_{i j}$ is shown to be true in the general case of arbitrary probability distribution of $a_{i j}$ [25]. This result is obtained by analysis of the high moments $M_{2 s_{n}}^{(n, n)}$ in the limit $n, s_{n} \rightarrow \infty$ with $s_{n}=O\left(n^{2 / 3}\right)$.

The high moments of large dilute random matrices $H^{\left(n, \rho_{n}\right)}(1.2)$ were studied in [12] in the asymptotic regime when $\rho=\rho_{n}=O\left(n^{\alpha}\right)$ with $2 / 3<\alpha<1$. It was proved that the limiting expression of the moments $M_{2 s_{n}}^{\left(n, \rho_{n}\right)}$ with $s_{n}=O\left(n^{2 / 3}\right)$ coincides with that of the moments of the Wigner random matrices $M_{2 s_{n}}^{(n, n)}$. This fact can be regarded as an evidence of the universal behavior of the local eigenvalue statistics for weakly dilute random matrices, i.e. when the dilution parameter $\rho$ is sufficiently large. In the present paper we study the opposite asymptotic regime of strongly dilute random matrices, i. e. when the dilution parameter $\rho_{n}$ tends to infinity as $n \rightarrow \infty$ but with much lower range than before, $\rho_{n}=O\left(n^{\alpha}\right)$ with $\alpha<1 / 5$. We show that in the limit

$$
n, \rho_{n} \rightarrow \infty, \rho_{n}=o\left(n^{1 / 5}\right), s_{n}=\left\lfloor\chi \rho_{n}\right\rfloor, \quad \chi>0,
$$

where $\lfloor x\rfloor$ is the integer part of $x$, the limiting expressions of $M_{2 s_{n}}^{\left(n, \rho_{n}\right)}$ are different from those obtained for the Wigner random matrix ensemble. This difference is due to the fact that the leading contribution to the moments $M_{2 s}^{(n, \rho)}$ in the asymptotic regime (1.4) is given by the trajectories that generalize in certain sense the Catalan numbers that describe the moments of the Wigner ensemble. Up to our knowledge, these trajectories of the new type were not considered before. Their combinatorial properties are of their own interest and this fact has motivated the work presented. In our studies, in particular, we obtain a number of explicit relations that were not known in the context of random matrices and plane rooted trees (see, for example, relation (1.9) below and formulas (5.3) and (5.4) of Section 5).

To make more compact the formulas we use, everywhere below we refer to the limiting transition (1.4) as $(n, s, \rho) \rightarrow \infty$. Our main result is given by the following statement.

Theorem 1.1. Assume that $V_{2}=1$ and that for all $1 \leq i \leq j$ the random variables $a_{i j}$ are bounded with probability 1 ,

$$
\left|a_{i j}\right| \leq U
$$

There exists a constant $\chi_{0}=\chi_{0}(U)>0$ such that for any given $0<\chi<\chi_{0}$ the following 
upper bound holds in the limit (1.4),

$$
\limsup _{(n, s, \rho) \rightarrow \infty} \frac{1}{n \mathrm{t}_{s}} M_{2 s_{n}}^{\left(n, \rho_{n}\right)} \leq 4 e^{16 \chi V_{4}}
$$

where

$$
\mathrm{t}_{s}=\frac{(2 s) !}{s !(s+1) !}, \quad s=0,1,2, \ldots
$$

are the Catalan numbers. The moments $M_{2 s_{n}}^{\left(n, \rho_{n}\right)}$ are given by the following asymptotic relation,

$$
M_{2 s_{n}}^{(n, \rho)}=n \hat{m}_{s_{n}}^{\left(\rho_{n}\right)}(1+o(1)), \quad(n, s, \rho) \rightarrow \infty,
$$

where the sequence $\left\{\hat{m}_{s}^{(\rho)}\right\}_{s \geq 0}$ is such that its generating function $F_{\rho}(z)=\sum_{s \geq 0} \hat{m}_{s}^{(\rho)} z^{s}$ verifies equation

$$
F_{\rho}(z)=1+z\left(F_{\rho}(z)\right)^{2}+\frac{z^{2} V_{4}}{\rho}\left(\frac{1}{1-z F_{\rho}(z)}\right)^{4}
$$

with the initial condition $\hat{m}_{0}^{(\rho)}=1$.

\section{Remarks}

1. We restrict the rate of $\rho_{n}$ by $n^{1 / 5}(1.4)$ not to overload the technical part of the paper. In fact, it follows from the proof of Theorem 1.1 that relations (1.6) and (1.8) can be obtained with (1.4) replaced by the limit when $\rho_{n} \rightarrow \infty$ and $\rho_{n}=o\left(n^{1 / 2}\right)$ (see formula (3.30) and the discussion that follows it). Moreover, one can expect that Theorem 1.1 remains valid in the asymptotic regime when $\rho_{n}=n^{\alpha}$ with $0<\alpha<2 / 3$. This asymptotic regime is complementary to the one studied in [12]. However, in the present paper we are aimed mostly at the lowest rates of $\rho_{n}$ having a particular interest in the asymptotic regime when $\rho_{n}=O(\log n), n \rightarrow \infty$.

2. In contrast to the technical restriction (1.4), it is not clear whether condition (1.5) can be relaxed, especially in the case of the asymptotic regime when $\rho_{n}=O(\log n), n \rightarrow \infty$ and $s_{n}=\left\lfloor\chi \rho_{n}\right\rfloor$. However, a part of the estimates that concerns the tree-type walks can be proved under considerably less restricted conditions than (1.5) (see relation (4.5) of Section 4 below).

3 . We will show that the numbers $\hat{m}_{s}^{(\rho)}$ are uniquely determined and verify the following upper bound (cf. (1.6)),

$$
\frac{1}{\mathrm{t}_{s}} \hat{m}_{s}^{(\rho)} \leq 4 e^{4 V_{4} s / \rho} .
$$

Therefore the generating function $F_{\rho}(z)(1.9)$ exists and is bounded in absolute value for any given $\rho$. Then it follows from (1.9) that the limiting function $f(z)=\lim _{\rho \rightarrow \infty} F_{\rho}(z)$ exists and verifies the following relation,

$$
f(z)=1+z(f(z))^{2} .
$$

This equation has a unique solution that determines the generating function of the Catalan numbers (1.7), $f(z)=\sum_{k \geq 0} \mathrm{t}_{k} z^{k}$.

4. Relation (1.8) can be rewritten in a more precise form. We will show that there exists a constant $C_{0}(U)>0$ such that the following relation holds

$$
\limsup _{(n, s, \rho) \rightarrow \infty} \frac{1}{n \mathrm{t}_{s}}\left(M_{2 s_{n}}^{\left(n, \rho_{n}\right)}-n \hat{m}_{s_{n}}^{\left(\rho_{n}\right)}\right) \leq \frac{C V_{4}}{\rho} e^{16 \chi V_{4}}
$$


in the limit $n, s_{n}, \rho_{n} \rightarrow \infty$ such that $s_{n}=\left\lfloor\chi \rho_{n}\right\rfloor, 0<\chi \leq \chi_{0}$ and $\rho_{n}=o\left(n^{1 / 6}\right)$. In fact, one can show that the left-hand side of relation (1.12) admits the asymptotic expansion in powers of $\rho$ and that the first terms of this expansion are given by relation

$$
\frac{1}{n \mathrm{t}_{s}} M_{2 s}^{(n, \rho)}=\frac{1}{\mathrm{t}_{s}}\left(\hat{m}_{s}^{(\rho)}+\frac{1}{\rho} R_{s}^{(1)}+o\left(\rho^{-1}\right)\right), \quad \rho \rightarrow \infty
$$

where

$$
R_{s}^{(1)}=\frac{4 V_{4}^{2}}{\rho} \frac{(2 s) !}{(s-4) !(s+4) !}+\frac{V_{6}}{\rho} \frac{(2 s) !}{(s-3) !(s+3) !}+o(\chi), \quad \chi \rightarrow 0
$$

(see the last part of Section 4).

5. As one will see, the numbers $\hat{m}_{s}^{(\rho)}, k \geq 0$ of (1.8) can be regarded as a generalization of the Catalan numbers $\mathrm{t}_{k}, k \geq 0$ in the following sense. The Catalan number $\mathrm{t}_{k}$ counts the half-plane rooted trees of $k$ edges. Regarding the chronological run over the such a tree, we get a closed walk of $2 k$ steps such that in its graph each edge is passed two times, in there and back directions. Using this terminology, we can say that $\hat{m}_{s}^{(\rho)}$ represents the sum of weighs of all closed walks of $2 s$ steps such that in their graphs each edge is passed either two or four times when counted in there and back directions. Also it is shown that in the corresponding graph the edges passed four times do not share a common vertex (see Section 4 for the rigorous definition of tree-type $(2,4)^{*}$-walks).

One of the consequences of this definition of numbers $\hat{m}_{s}^{(\rho)}$ is given by the following lower bound (see formula (5.3) of Section 5 below),

$$
\hat{m}_{s}^{(\rho)} \geq \mathrm{t}_{s}+\frac{V_{4}}{\rho} \cdot \frac{(2 s) !}{(s-2) !(s+2) !}
$$

where the last fraction represents the number of closed tree-type walks of $2 s$ steps such that their graphs contain exactly one edge passed four times. Then in the limiting transition (1.4) we get the following lower bound,

$$
\liminf _{(n, s, \rho) \rightarrow \infty} \frac{1}{\mathrm{t}_{s}} \hat{m}_{s}^{(\rho)} \geq\left(1+\chi V_{4}\right) .
$$

Let us discuss relations of the results of Theorem 1.1 with the spectral properties of large dilute random matrices $H^{(n, \rho)}(1.2)$. Regarding the ordered family of real eigenvalues of the matrix $H^{(n, \rho)}(\omega), \lambda_{1}^{(n, \rho)}(\omega) \leq \cdots \leq \lambda_{n}^{(n, \rho)}(\omega)$, we denote its spectral norm by $\lambda_{\max }^{(n, \rho)}(\omega)$,

$$
\left\|H^{(n, \rho)}(\omega)\right\|=\lambda_{\max }^{(n, \rho)}(\omega)=\max \left\{\left|\lambda_{1}^{(n, \rho)}(\omega)\right|,\left|\lambda_{n}^{(n, \rho)}(\omega)\right|\right\} .
$$

The well-known analog of the Chebyshev inequality for the deviation probability

$$
P\left(\lambda_{\max }^{(n, \rho)} \geq 2(1+\varepsilon)\right) \leq \frac{1}{(2(1+\varepsilon))^{2 s}} \mathbf{E}\left(\sum_{j=1}^{n}\left(\lambda_{j}^{(n, \rho)}\right)^{2 s}\right)=\frac{1}{(2(1+\varepsilon))^{2 s}} M_{2 s}^{(n, \rho)}
$$

allows us to deduce from estimate (1.6) the following upper bound valid for all $n \geq n_{0}$ with some $n_{0}$

$$
P\left(\lambda_{\max }^{(n, \rho)} \geq 2(1+\varepsilon)\right) \leq 6 e^{16 V_{4} \chi} \frac{n \mathrm{t}_{s}}{(2(1+\varepsilon))^{2 s}} .
$$


Applying the Stirling formula to the Catalan numbers $t_{s}$ (1.7), we get inequality

$$
P\left(\lambda_{\max }^{(n, \rho)} \geq 2(1+\varepsilon)\right) \leq 4 e^{16 V_{4} \chi} \frac{n}{s^{3 / 2}(1+\varepsilon)^{2 s}} .
$$

Remembering that $s_{n}=\left\lfloor\chi \rho_{n}\right\rfloor$, we deduce from (1.16) that if the sequence $\left(\rho_{n}\right)_{n \geq 1}$ is such that $\rho_{n} / \log n \rightarrow \infty$ as $n \rightarrow \infty$, then for any given $\varepsilon>0$

$$
\sum_{n \geq n_{0}} P\left(\lambda_{\max }^{\left(n, \rho_{n}\right)} \geq 2(1+\varepsilon)\right)<\infty
$$

Relation (1.17) means that $P\left(\lim \sup _{n \rightarrow \infty} \lambda_{\max }^{\left(n, \rho_{n}\right)} \leq 2\right)=1$ in this asymptotic regime.

Taking into account the fact that the semicircle law is valid for the eigenvalue distribution of $H^{\left(n, \rho_{n}\right)}$ in the limit $n, \rho_{n} \rightarrow \infty$ [13], it is not hard to conclude that with probability 1 ,

$$
\lim _{n \rightarrow \infty}\left\|H^{\left(n, \rho_{n}\right)}\right\|=2, \quad \frac{\rho_{n}}{\log n} \rightarrow \infty, n \rightarrow \infty .
$$

This statement slightly improves our earlier results [9], where the convergence of $\lambda_{\max }^{\left(n, \rho_{n}\right)}$ to 2 has been proved in the asymptotic regime when $\rho_{n}=(\log n)^{1+\delta}$ with given $\delta>0$. Let us note however, that in the present article we prove Theorem 1.1 and (1.18) under condition (1.5) that is more restrictive than those imposed in paper [9].

Returning to inequalities (1.15) and (1.16), we can rewrite them in the following form

$$
\Psi_{n}(x)=P\left(\lambda_{\max }^{(n, \rho)} \geq 2\left(1+\frac{x}{h_{n}}\right)\right) \leq \frac{4 n}{s^{3 / 2}} \exp \left\{2 \chi\left(8 V_{4}-\frac{x \rho_{n}}{h_{n}}\right)\right\} .
$$

The right-hand side of (1.19) shows that in the limit of infinite $n$, the probability to find eigenvalues of $H^{\left(n, \rho_{n}\right)}$ outside of the interval $\left(-2\left(1+x / h_{n}\right), 2\left(1+x / h_{n}\right)\right.$ goes to zero provided $h_{n}$ is much smaller than $\rho_{n}(\ln n)^{-1}$, i.e. the length of the corresponding interval is larger than $(\log n) / \rho_{n}$.

Neglecting the first factor in the right-hand side of (1.19), we can observe that to obtain a non-trivial and non-zero limit $\Psi_{n}(x) \rightarrow \Psi(x)$, it is natural to consider the scaling parameter $h_{n}$ of the left-hand side of (1.19) to be of the order $O\left(\rho_{n}\right)$. This reasoning could be compared with the result widely known as the Tracy-Widom distribution for the maximal eigenvalue of Gaussian Unitary (and Orthogonal) Ensembles of random matrices of the form (1.1), where the limiting expression of $\Psi_{n}(x)$ is explicitly determined with the scaling factor $h_{n}=n^{-2 / 3}$ [27] (see also monographs $[1,18]$ ).

It should be stressed that in papers $[25,26]$, the existence of a non-trivial non-zero limit of the moments $\lim _{n \rightarrow \infty} M_{2 s_{n}}^{(n, n)}=\mathcal{M}(\tau), s_{n}=\left\lfloor\tau n^{2 / 3}\right\rfloor$ of the Wigner random matrices $A_{n}(1.1)$ is shown to imply the Tracy-Widom law for the maximal eigenvalue distribution in the case of arbitrarily distributed matrix entries $a_{i j}$. The limiting expression $\mathcal{M}(\tau)$ being independent from the the particular values of the moments $V_{2 l}, l \geq 2$ of $a_{i j}$, the result of [25] means a wide universality of the Tracy-Widom law for large random matrices.

In paper [12] we have proved that the same limiting expression $\mathcal{M}(\tau)=\lim _{n \rightarrow \infty} M_{2 s_{n}}^{\left(n, \rho_{n}\right)}$, $s_{n}=\left\lfloor\tau n^{2 / 3}\right\rfloor$ is valid for the ensemble of dilute random matrices (1.2) in the asymptotic 
regime of weak dilution, $\rho_{n} / n^{2 / 3} \rightarrow \infty$. In contrast, when the dilution becomes moderate, i.e. when $\rho_{n}=\zeta n^{2 / 3}$ with given $\zeta>0$, the estimate from below (see [12], Theorem 7.1)

$$
\liminf _{n \rightarrow \infty} M_{2 s_{n}}^{\left(n, \rho_{n}\right)} \geq \frac{4 V_{4}}{\zeta \sqrt{\pi \tau}} e^{-e \tau^{3}}
$$

shows that the universality of the Tracy-Widom distribution discussed above cannot hold in this asymptotic regime. This is because the fourth moment $V_{4}$ enters explicitly into the lower bound (1.20) while this is not so in the case of non-diluted or weakly diluted Wigner random matrices according to the statements of $[25,26]$.

Returning to the case of the strong dilution when $\rho_{n}=o\left(n^{1 / 5}\right)(1.4)$, one can see that the situation becomes even worse than that of (1.20) because the sequence of moments $M_{2 s_{n}}^{\left(n, \rho_{n}\right)}$ diverges exponentially with respect to $s_{n} / \rho_{n}$, i.e. whenever $s_{n}$ is of the order greater than $\rho_{n}$ as $n \rightarrow \infty$. This is because the expression in the right-hand side of the upper bound (1.10) turns out to be the lower one with the numerical constants tuned up (see a conjecture on the numbers $\mathcal{N}_{s}^{(p, 2)}$ at the end of the sub-section 5.2). The exponential divergence of $M_{2 s_{n}}^{\left(n, \rho_{n}\right)}$ can be regarded as one more argument to the conjecture that the scaling parameter $h_{n}(1.19)$ at the edge of the spectrum of large strongly diluted random matrices has to be switched from $n^{-2 / 3}$ to $\rho_{n}^{-1}$. We postpone the study of this problem to subsequent publications.

\section{Trajectories, walks and graphical representations}

In this section we describe the main components of the method we develop to study the high moments of dilute random matrices $H^{(n, \rho)}(1.2)$. In the pioneering works of E. Wigner (see e.g. [28]), it was proposed to consider the moments $M_{2 s}$ of random matrices as a weighted sum over paths of $2 s$ steps. In the case of dilute random matrices, we can write that

$$
M_{2 s}^{(n, \rho)}=\sum_{i=1}^{n} \mathbf{E}\left(H^{(n, \rho)}\right)_{i i}^{2 s}=\sum_{\mathcal{I}_{2 s} \in \mathbb{I}_{2 s}(n)} \Pi_{a, b}\left(\mathcal{I}_{2 s}\right)=\sum_{\mathcal{I}_{2 s} \in \mathbb{I}_{2 s}(n)} \Pi_{a}\left(\mathcal{I}_{2 s}\right) \Pi_{b}\left(\mathcal{I}_{2 s}\right),
$$

where the sequence $\mathcal{I}_{2 s}=\left(i_{0}, i_{1}, \ldots, i_{2 s-1}, i_{0}\right), i_{k} \in\{1,2, \ldots, n\}$ is regarded as a closed path of $2 s$ steps $\left(i_{t-1}, i_{t}\right)$ with the discrete time $t \in[0,2 s]$. We will also say that $\mathcal{I}_{2 s}$ is a trajectory of $2 s$ steps. The set of all possible trajectories of $2 s$ steps over $\{1, \ldots, n\}$ is denoted by $\mathbb{I}_{2 s}(n)$. The weights $\Pi_{a}\left(\mathcal{I}_{2 s}\right)$ and $\Pi_{b}\left(\mathcal{I}_{2 s}\right)$ are determined as the mathematical expectations of the products of corresponding random variables,

$$
\Pi_{a}\left(\mathcal{I}_{2 s}\right)=\mathbf{E}\left(a_{i_{0} i_{1}} \cdots a_{i_{2 s-1} i_{0}}\right), \quad \Pi_{b}\left(\mathcal{I}_{2 s}\right)=\mathbf{E}\left(b_{i_{0} i_{1}} \cdots b_{i_{2 s-1} i_{0}}\right) .
$$

Here and below, we omit the superscripts in $b_{i j}^{(n, \rho)}$ when no confusion can arise.

In papers $[22,23]$ a deep and powerful generalization of the E. Wigner's approach was proposed by Ya. Sinai and A. Soshnikov to study the high moments of random matrices. Somehow different point of view has been developed to consider the ensembles of dilute random matrices $[9,12,15]$. The difference between these two approaches is dictated by the fact that the leading contribution to the moments $M_{2 s}^{(n, \rho)}$ of the dilute random matrices $H^{(n, \rho)}(1.2)$ is given by those trajectories $\mathcal{I}_{2 s}$ that have a vanishing weight in the case of non-diluted Wigner random matrices $A_{n}(1.1)$ studied in $[22,23]$. In the first part of the present paper we use a 
combination of these two approaches to study the terms that give the vanishing contribution to the moments of strongly diluted random matrices (see Section 3). On the second stage we develop a new method that allows us to prove that in the limit of infinitely increasing dimension, non-zero contribution to the moments $M_{2 s}^{(n, \rho)}$ is given by the new kind of tree-type walks such that their weight contains the factors $V_{2}$ and $V_{4}$ only (see Section 4). We refer to this kind of walks as to $(2,4)$-walks. To determine rigorously the corresponding classes of trajectories, we need to describe briefly the fundamental notions of the methods developed in papers mentioned above.

Regarding a trajectory $\mathcal{I}_{2 s}$ one can determine a walk

$$
\mathcal{W}_{2 s}=\mathcal{W}_{2 s}^{\left(\mathcal{I}_{2 s}\right)}=\{\mathcal{W}(t), t \in[0,2 s]\}, \quad \text { where } \quad[0,2 s]=\{0,1,2, \ldots, 2 s\},
$$

that we define as a sequence of $2 s+1$ symbols (or equivalently, letters) from an ordered alphabet, say $\mathcal{A}=\left\{\alpha_{1}, \alpha_{2}, \ldots\right\}$. The walk $\mathcal{W}_{2 s}^{\left(\mathcal{I}_{2 s}\right)}$ is constructed with the help of the following rules of recurrence [15]. Given a trajectory $\mathcal{I}_{2 s}$, we write that $\mathcal{I}_{2 s}(t)=i_{t}, t \in[0,2 s]$ and consider a subset $\mathbb{U}\left(\mathcal{I}_{2 s} ; t\right)=\left\{\mathcal{I}_{2 s}\left(t^{\prime}\right), 0 \leq t^{\prime} \leq t\right\} \subseteq\{1,2, \ldots, n\}$. We denote by $\left|\mathbb{U}\left(\mathcal{I}_{2 s} ; t\right)\right|$ its cardinality. Then

1) $\mathcal{W}_{2 s}(0)=\alpha_{1}$;

2) if $\mathcal{I}_{2 s}(t+1) \notin \mathbb{U}\left(\mathcal{I}_{2 s} ; t\right)$, then $\mathcal{W}_{2 s}(t+1)=\alpha_{\left|\mathbb{U}\left(\mathcal{I}_{2 s} ; t\right)\right|+1}$;

if there exists $t^{\prime} \leq t$ such that $\mathcal{I}_{2 s}(t+1)=\mathcal{I}_{2 s}\left(t^{\prime}\right)$, then $\mathcal{W}_{2 s}(t+1)=\mathcal{W}_{2 s}\left(t^{\prime}\right)$.

For example, $\mathcal{I}_{16}=(5,2,7,9,7,1,5,2,7,9,7,2,7,2,7,1,5)$ produces the walk

$$
\mathcal{W}_{16}=\left(\alpha_{1}, \alpha_{2}, \alpha_{3}, \alpha_{4}, \alpha_{3}, \alpha_{5}, \alpha_{1}, \alpha_{2}, \alpha_{3}, \alpha_{4}, \alpha_{3}, \alpha_{2}, \alpha_{3}, \alpha_{2}, \alpha_{3}, \alpha_{5}, \alpha_{1}\right)
$$

We say that the pair $\left(\mathcal{W}_{2 s}(t-1), \mathcal{W}_{2 s}(t)\right)$ represents the $t$-th step of the walk $\mathcal{W}_{2 s}$ and that $\alpha_{1}$ represents the root of the walk $\mathcal{W}_{2 s}$.

Taking two trajectories $\mathcal{I}_{2 s}^{\prime}$ and $\mathcal{I}_{2 s}^{\prime \prime}$ such that $\mathcal{W}_{2 s}^{\left(\mathcal{I}_{2 s}^{\prime}\right)}=\mathcal{W}_{2 s}^{\left(\mathcal{I}_{2 s}^{\prime \prime}\right)}=\mathcal{W}_{2 s}$, we say that they are equivalent, $\mathcal{I}_{2 s}^{\prime} \sim \mathcal{I}_{2 s}^{\prime \prime}$. We denote by $\mathcal{C}_{\mathcal{W}_{2 s}}$ the corresponding class of equivalence. It is clear that

$$
\left|\mathcal{C}_{\mathcal{W}_{2 s}}\right|=n(n-1) \cdots\left(n-\left|\mathbb{U}\left(\mathcal{I}_{2 s} ; 2 s\right)\right|+1\right) .
$$

Given $\mathcal{W}_{2 s}$, one can introduce a graphical representation $g\left(\mathcal{W}_{2 s}\right)=\left(\mathbb{V}_{g}, \mathbb{E}_{g}\right)$ that can be considered as a kind of multigraph with the set of vertices $\mathbb{V}_{g}=\left\{\alpha_{1}, \ldots, \alpha_{\left|\mathbb{U}\left(\mathcal{I}_{2 s} ; 2 s\right)\right|}\right\}$ and the set $\mathbb{E}_{g}$ of $2 s$ oriented edges (or equivalently, arcs) labelled by $t \in\{1, \ldots, 2 s\}$. To describe the properties of $g\left(\mathcal{W}_{2 s}\right)$ in general situations, we will use greek letters $\alpha, \beta, \gamma, \ldots$ instead of the symbols from $\mathcal{A}$. In this case, the root of the walk will be denoted by $\varrho$. In what follows, we refer to $g\left(\mathcal{W}_{2 s}\right)$ simply as to the graph of the walk $\mathcal{W}_{2 s}$. If $\mathcal{W}_{2 s}(t)=\gamma$, we say that $\gamma$ is the value of the walk $\mathcal{W}_{2 s}$ at the instant of time $t$.

Let us define the current multiplicity of the couple of vertices $\{\beta, \gamma\}, \beta, \gamma \in \mathbb{V}_{g}$ up to the instant $t$ by the following variable

$\mathfrak{m}_{\mathcal{W}}^{(\{\beta, \gamma\})}(t)=\#\left\{t^{\prime} \in[1, t]:\left(\mathcal{W}_{2 s}\left(t^{\prime}-1\right), \mathcal{W}_{2 s}\left(t^{\prime}\right)\right)=(\beta, \gamma)\right.$ or $\left.\left(\mathcal{W}_{2 s}\left(t^{\prime}-1\right), \mathcal{W}_{2 s}\left(t^{\prime}\right)\right)=(\gamma, \beta)\right\}$

and say that $\mathfrak{m}_{\mathcal{W}}^{(\{\beta, \gamma\})}(2 s)$ represents the total multiplicity of the couple $\{\beta, \gamma\}$.

The probability law of $a_{i j}$ being symmetric, the weight of $\mathcal{I}_{2 s}$ (1.16) is non-zero if and only if $\mathcal{I}_{2 s}$ is such that in the corresponding graph of the walk $\mathcal{W}_{2 s}^{\left(\mathcal{I}_{2 s}\right)}$ each couple $\{\alpha, \beta\}$ has an even multiplicity $\mathfrak{m}_{\mathcal{W}}^{(\{\alpha, \beta\})}(2 s)=0(\bmod 2)$. We refer to the walks of such trajectories as to the 
even closed walks [22] and denote by $\mathbb{W}_{2 s}$ the set of all possible even closed walks of $2 s$ steps. In what follows, we consider the even closed walks only and refer to them simply as to the walks.

It is natural to say that the pair $\left(\mathcal{W}_{2 s}(t-1), \mathcal{W}_{2 s}(t)\right)=\mathfrak{s}_{t}$ represents the step of the walk number $t$. Given $\mathcal{W}_{2 s} \in \mathbb{W}_{2 s}$, we say that the instant of time $t$ is marked [22] if the couple $\{\alpha, \beta\}=\left\{\mathcal{W}_{2 s}(t-1), \mathcal{W}_{2 s}(t)\right\}$ has an odd current multiplicity $\mathfrak{m}_{\mathcal{W}}^{(\{\alpha, \beta\})}(t)=1(\bmod 2)$. We also say that the corresponding step $\mathfrak{s}_{t}$ and the edge $e_{t}$ of $g\left(\mathcal{W}_{2 s}\right)$ are marked. All other steps and edges are called the non-marked ones. Regarding a collection of the marked edges $\overline{\mathbb{E}}_{g}$ of $g\left(\mathcal{W}_{2 s}\right)$, we consider a multigraph $\bar{g}_{s}=\left(\overline{\mathbb{V}}_{g}, \overline{\mathbb{E}}_{g}\right)$. Clearly, $\overline{\mathbb{V}}_{g}=\mathbb{V}_{g}$ and $\left|\overline{\mathbb{E}}_{g}\right|=s$. It is useful to keep the time labels of the edges $\overline{\mathbb{E}}_{g}$ as they are in $\mathbb{E}_{g}$. Given two edges $e^{\prime}=e_{t^{\prime}}$ and $e^{\prime \prime}=e_{t^{\prime \prime}}$ such that $t^{\prime}<t^{\prime \prime}$, we write that $e^{\prime}<e^{\prime \prime}$. Sometimes we denote $t^{\prime}=t\left(e^{\prime}\right)$.

In general, $\bar{g}\left(\mathcal{W}_{2 s}\right)$ is a multigraph with multiple edges. Replacing the multiple edge by a simple one, we get a new graph that we refer to as the skeleton $S_{\bar{g}}$ of the graph $\bar{g}$.

Any even closed walk $\mathcal{W}_{2 s} \in \mathbb{W}_{2 s}$ generates a sequence $\theta_{2 s}$ of $s$ marked and $s$ non-marked instants. Corresponding sequence of $2 s$ signs + and - is known to encode a Dyck path of $2 s$ steps. We denote by $\theta_{2 s}=\theta\left(\mathcal{W}_{2 s}\right)$ the Dyck path of $\mathcal{W}_{2 s}$ and say that $\theta\left(\mathcal{W}_{2 s}\right)$ represents the Dyck structure of $\mathcal{W}_{2 s}$.

Let us denote by $\Theta_{2 s}$ the set of all Dyck paths of $2 s$ steps. It is known that $\Theta_{2 s}$ is in one-by-one correspondence with the set of all half-plane rooted trees $\mathcal{T}_{s} \in \mathbb{T}_{s}$ constructed with the help of $s$ edges. The correspondence between $\Theta_{2 s}$ and $\mathbb{T}_{s}$ can be established with the help of the chronological run $\mathfrak{R}$ over the edges of $\mathcal{T}_{s}$. It is known that the cardinalities of $\mathbb{T}_{s}$, $s=0,1,2, \ldots$ are given by the Catalan numbers,$\left|\mathbb{T}_{s}\right|=\mathrm{t}_{s}(1.7)$. We refer to the elements of $\mathbb{T}_{s}$ as to the Catalan trees. We consider the edges of the tree $\mathcal{T}_{s}$ as the oriented ones in the direction away from the root of $\mathcal{T}_{s}$.

Given a Catalan tree $\mathcal{T}_{s} \in \mathbb{T}_{s}$, one can label its vertices with the help of letters of $\mathcal{A}$ according to $\mathfrak{R}_{\mathcal{T}}$. The root vertex gets the label $\alpha_{1}$ and each new vertex that has no label is labelled by the next in turn letter. We denote the walk obtained by $\mathcal{W}_{2 s}\left[\mathcal{T}_{s}\right]$ and the corresponding Dyck path $\theta_{2 s}=\theta\left(\stackrel{\circ}{\mathcal{W}}_{2 s}\right)$ will be denoted also as $\theta_{2 s}=\theta\left(\mathcal{T}_{s}\right)$.

Any Dyck path $\theta_{2 s}$ generates a sequence $\left(\xi_{1}, \xi_{2}, \ldots, \xi_{s}\right), \xi_{i} \in\{1,2, \ldots, 2 s-1\}$ such that each step $\mathfrak{s}_{\xi_{i}}, 1 \leq i \leq s$ of $\mathcal{W}_{2 s}\left[\theta_{2 s}\right]$ is marked. We denote this sequence by $\Xi_{s}=\Xi\left(\theta_{2 s}\right)$. Given $\Xi_{s}$ and $\tau \in[1, s]$, one can uniquely reconstruct $\theta_{2 s}$ and find corresponding instant of time $\xi_{\tau} \in\{1, \ldots, 2 s-1\}$. We will say that the interval $[1, s]$ represents the $\tau$-marked instants or instants of marked time that varies from 1 to $s$; sometimes we will simply say that $\tau \in[1, s]$ is the marked instant when no confusion can arise.

Given a walk $\mathcal{W}_{2 s}$ and a letter $\beta$ such that $\beta \in \mathbb{V}_{g}\left(\mathcal{W}_{2 s}\right)$, we say that the instant of time $t^{\prime}$ such that $\mathcal{W}_{2 s}\left(t^{\prime}\right)=\beta$ represents an arrival $\mathfrak{a}$ at $\beta$. If $t^{\prime}$ is marked, we will say that the corresponding arrival $\mathfrak{a}(\beta)$ is the marked arrival at $\beta$. In $\mathcal{W}_{2 s}$, there can be several marked arrival instants of time at $\beta$ that we denote by $1 \leq t_{1}^{(\beta)}<\cdots<t_{N}^{(\beta)}$. For any non-root vertex $\beta$, we have $N=N_{\beta} \geq 1$. The first arrival instant of time $\beta$ is always the marked one. We can say that $\beta$ is created at this instant of time. To unify the description, we assume that the root vertex $\varrho$ is created at the zero instant of time $t_{1}^{(\rho)}=0$ and add the corresponding zero marked instant to the list of the marked arrival instants at $\varrho$.

If $N_{\beta} \geq 2$, then we say that the $N$-plet $\left(t_{1}^{(\beta)}, \ldots, t_{N}^{(\beta)}\right)$ of marked arrival instants of time rep- 
resents the self-intersection of $\mathcal{W}_{2 s}, \beta$ is the vertex of self-intersection, and this self-intersection is of the degree $N$ [22]. We say that the self-intersection degree $\varkappa(\beta)$ is equal to $N$ and denote this by $\varkappa(\beta)=N_{\beta}$. If $N_{\beta}=1$, then we will say that $\varkappa(\beta)=1$.

Finally, let us consider a vertex $\beta$ and a collection of the marked edges of the form $\left(\beta, \alpha_{i}\right)$. We say that this collection is the exit cluster of $\beta$ and denote it by $\Delta(\beta)$,

$$
\Delta(\beta)=\Delta_{\mathcal{W}}(\beta)=\left\{e \in \overline{\mathbb{E}}\left(\mathcal{W}_{2 s}\right): e=\left(\beta, \alpha_{i}\right)\right\} .
$$

Sometimes we will say that $\Delta(\beta)$ is given by the collection of corresponding vertices $\alpha_{i}$.

Finally, let us determine the tree-type walks and $(2,4)$-walks we will study. Given $\mathcal{W}_{2 s}$, we can say that it is of the tree-type form if $\bar{g}\left(\mathcal{W}_{2 s}\right)$ has no cycles, i.e. when $\bar{g}\left(\mathcal{W}_{2 s}\right)$ represents a tree when the multiple edges are considered as the single ones. If $\bar{g}\left(\mathcal{W}_{2 s}\right)$ has at least one cycle, we can say that corresponding $\mathcal{W}_{2 s}$ is not of the tree-type structure. Regarding a walk of the tree-type form $\hat{\mathcal{W}}_{2 s}$, we will say that it is a $(2,4)$-walk if the weight of the corresponding trajectory $\Pi\left(\mathcal{I}_{2 s}\right)(2.2)$ contains the factors $V_{2}$ and $V_{4}$ only. If the $(2,4)$-walk is such that in its graph the multiple edges with the weights $V_{4}$ have no vertices in common, we will say that this walk is the $(2,4)^{*}$-walk.

To complete this section, let us note that the number of the tree-type walks of $2 s$ steps whose weight is given by $V_{2}^{s}$ is given by the Catalan numbers $\mathrm{t}_{s}$ (1.7). It follows from (1.1) that these numbers verify the following recurrent relation

$$
\mathrm{t}_{k}=\sum_{j=0}^{k-1} \mathrm{t}_{k-1-j} \mathrm{t}_{j}, \quad k \geq 1
$$

with the initial condition $t_{0}=1$. In the studies of the $(2,4)$-walks, we find a number of generalizations of relations (1.7) and (2.6).

\section{Walks of non-tree type}

\subsection{Classification of vertices and weights of walks}

Given a walk $\mathcal{W}_{2 s}$ and its graph $\bar{g}\left(\mathcal{W}_{2 s}\right)=\left(\mathbb{V}_{g}, \overline{\mathbb{E}}_{g}\right)$ and consider two vertices $\alpha$ and $\beta$. We denote by $\mathcal{E}_{\{\alpha, \beta\}}$ the collection of all marked edges of the form $(\alpha, \beta)$ or $(\beta, \alpha)$ of $\bar{g}\left(\mathcal{W}_{2 s}\right)$ and determine the minimal edge $\tilde{e}=\min \left\{e: e \in \mathcal{E}_{\{\alpha, \beta\}}\right\}$. Let us assume that $\tilde{e}$ is of the form $(\alpha, \beta)$.

If the multi-edge $\mathcal{E}_{\{\alpha, \beta\}}$ contains the edge $e_{1}$ of the first arrival at $\beta, e_{1}=e\left(\mathfrak{a}_{1}(\beta)\right)$, then $\tilde{e}=e_{1}$ and we say that this edge $\tilde{e}=(\alpha, \beta)$ creates $\beta$ and that $\tilde{e}$ is the base edge of $\beta$ or simply the base edge.

Let us consider the edge of the second arrival at $\beta, e_{2}=e\left(\mathfrak{a}_{2}(\beta)\right)$. If $e_{2}=(\alpha, \beta)$, then we color it in green and say that $\beta$ is the green p-vertex.

Let us consider the edge $e_{2}=e\left(\mathfrak{a}_{2}\right)=(\gamma, \beta)$ of the second arrival at $\beta$ such that $\gamma \neq \beta$. If $e_{2}$ is the minimal edge of the multi-edge $\mathcal{E}_{\{\beta, \gamma\}}$, then we say that $\beta$ is the blue r-vertex and color $e_{2}$ in blue.

Let us consider the case when $\tilde{e}=\min \left\{e: e \in \mathcal{E}_{\{\gamma, \beta\}}\right\}=(\beta, \gamma)$. If $\tilde{e}$ is the edge of the first or the second arrival at $\gamma, \tilde{e}=\mathfrak{a}_{i}(\gamma)$ with $i=1$ or $i=2$, then we color $e_{2}=(\gamma, \beta)$ in red and say that $\beta$ is the red q-vertex. If $\tilde{e}=\mathfrak{a}_{j}(\gamma)$ with $j \geq 3$, then we color $e_{2}=(\gamma, \beta)$ in blue and consider $\beta$ as the blue $r$-vertex. 
It is not hard to see that all edges of the second arrival to one or another vertex are colored and that their colors are uniquely determined. All remaining edges of $\bar{g}\left(\mathcal{W}_{2 s}\right)$ that are not the base or the color ones are referred as to the grey u-edges.

Lemma 3.1. Let $\mathcal{I}_{2 s}$ be such that the graph of its walk $\mathcal{W}_{2 s}=\mathcal{W}\left(\mathcal{I}_{2 s}\right)$ contains $r$ blue $r$-vertices, $p$ green p-vertices, q red q-vertices. Also we assume that $\bar{g}\left(\mathcal{W}_{2 s}\right)$ has u grey u-edges. Then the weight of $\mathcal{I}_{2 s}$ (2.2) is bounded as follows

$$
\Pi_{a}\left(\mathcal{I}_{2 s}\right) \Pi_{b}\left(\mathcal{I}_{2 s}\right)=\Pi_{a}\left(\mathcal{W}_{2 s}\right) \Pi_{b}\left(\mathcal{W}_{2 s}\right) \leq\left(\frac{V_{2}^{2}}{n^{2}}\right)^{r}\left(\frac{V_{2} U^{2}}{n \rho}\right)^{p+q}\left(\frac{U^{2}}{\rho}\right)^{u}\left(\frac{V_{2}}{n}\right)^{s-u-2(r+p+q)} .
$$

Remark. To make the statements of the present section and their proofs clearer, we keep the factors $V_{2}$ as they are remembering that $V_{2}=1$.

Proof of Lemma 3.1. The weight of the walk $\Pi_{a, b}\left(\mathcal{W}_{2 s}\right)=\Pi_{a}\left(\mathcal{W}_{2 s}\right) \Pi_{b}\left(\mathcal{W}_{2 s}\right)$ is given by the product of weights of all existing multi-edges $\Pi_{a, b}\left(\mathcal{E}_{\{\delta, \epsilon\}}\right)$. It is easy to see that the weight of the multi-edge can be estimated as follows,

$$
\Pi_{a, b}\left(\mathcal{E}_{\{\delta, \epsilon\}}\right) \leq\left(\frac{V_{2}}{n}\right)^{I_{b, r}}\left(\frac{U^{2}}{\rho}\right)^{I_{r, p}+I_{q}+u(\mathcal{E})},
$$

where $I_{b, r}=1$ if the minimal edge $\tilde{e}$ of $\mathcal{E}_{\{\delta, \epsilon\}}$ is either the base one or the blue one and zero otherwise, $I_{r, p}$ is equal to one if $\mathcal{E}_{\{\delta, \epsilon\}}$ contains a green edge and zero otherwise, $I_{q}$ is equal to one if $\mathcal{E}_{\{\delta, \epsilon\}}$ contains a red edge and zero otherwise and $u(\mathcal{E})=u\left(\mathcal{E}_{\{\delta, \epsilon\}}\right)$ represents the number of the grey edges in $\mathcal{E}_{\{\delta, \epsilon\}}$. Due to this factorization, the weight of the walk can be estimated by the product of factors $V_{2} / n$ and $U^{2} / \rho$ that can be rearranged in the product with respect to all vertices of the graph $g\left(\mathcal{W}_{2 s}\right)$. This can be done by attributing the weights $V_{2} / n$ to all base and blue edges of the graph $\bar{g}\left(\mathcal{W}_{2 s}\right)$ and the weights $U^{2} / \rho$ to all green, red and grey edges of $\bar{g}\left(\mathcal{W}_{2 s}\right)$ and by attributing to each vertex $\beta$ the product of weights of all edges that enter $\beta$. It is clear that any color vertex has exactly one edge of the second arrival that is of the same color as the vertex. This observation completes the proof of Lemma 3.1.

\subsection{Tree-type walks and walks of non-tree type}

Given a walk $\mathcal{W}_{2 s}$, we say that is it a tree-type walk if its graph $\bar{g}\left(\mathcal{W}_{2 s}\right)$ does not contain any blue $r$-vertex. We denote by $\hat{\mathbb{W}}_{2 s}$ a collection of tree-type walks. If $\mathcal{W}_{2 s}$ is such that its graph $\bar{g}\left(\mathcal{W}_{2 s}\right)$ contains at least one blue $r$-vertex, then we say that $\mathcal{W}_{2 s}$ is of non-tree type. We denote a collection of all non-tree-type walks by $\tilde{\mathbb{W}}_{2 s}$.

The following simple statement plays an important role in our studies.

Lemma 3.2. If $\mathcal{W}_{2 s}$ is such that its graphical representation $g\left(\mathcal{W}_{2 s}\right)$ has at least one red $q$-vertex, then $g\left(\mathcal{W}_{2 s}\right)$ contains at least one blue r-vertex.

We prove Lemma 3.2 in Section 5. Regarding the example walk $\mathcal{W}_{16}(2.3)$, we see that its graphical presentation contains two vertices of the self-intersection degree 2 (these are $\alpha_{1}$ and $\alpha_{4}$ ) and one vertex $\alpha_{2}$ of the self-intersection degree 3 . Among vertices of $\bar{g}\left(\mathcal{W}_{16}\right)$, there is one $p$-vertex $\alpha_{4}$ and one $q$-vertex $\alpha_{2}$. The root vertex $\alpha_{1}$ has one blue edge of the (mute) 
first arrival and one blue edge $e(6)$ of the second distinct arrival. So, the root vertex $\alpha_{1}$ is the blue $r$-vertex and $\mathcal{W}_{16}$ is of non-tree type.

According to definitions, we can rewrite relation (2.1) in the form

$$
M_{2 s}^{(n, \rho)}=\tilde{\mathcal{Z}}_{2 s}^{(n, \rho)}+\hat{\mathcal{Z}}_{2 s}^{(n, \rho)}
$$

where

$$
\tilde{\mathcal{Z}}_{2 s}^{(n, \rho)}=\sum_{\mathcal{I}_{2 s}: \mathcal{W}^{\left(\mathcal{I}_{2 s}\right)} \in \tilde{\mathbb{W}}_{2 s}} \Pi_{a}\left(\mathcal{I}_{2 s}\right) \Pi_{b}\left(\mathcal{I}_{2 s}\right)
$$

and

$$
\hat{\mathcal{Z}}_{2 s}(n, \rho)=\sum_{\mathcal{I}_{2 s}: \mathcal{W}^{\left(\mathcal{I}_{2 s}\right)} \in \hat{\mathbb{W}}_{2 s}} \Pi_{a}\left(\mathcal{I}_{2 s}\right) \Pi_{b}\left(\mathcal{I}_{2 s}\right) .
$$

The following statements represents the main technical result of this section.

Theorem 3.1. Under conditions of Theorem 1.1, the following relation holds

$$
\tilde{\mathcal{Z}}_{2 s_{n}}(n, \rho)=o\left(n \mathrm{t}_{s_{n}}\right), \quad(n, s, \rho) \rightarrow \infty .
$$

Remark. Observing that all terms of the right-hand side of the definition of $\hat{\mathcal{Z}}_{2 s}^{(n, \rho)}$ are non-negative, we conclude that $\hat{\mathcal{Z}}_{2 s}(n, \rho) \geq n \mathrm{t}_{s} V_{2}^{s}=n \mathrm{t}_{s}$. Therefore relation (3.3) implies the asymptotic estimate

$$
\tilde{\mathcal{Z}}_{2 s}(n, \rho)=o\left(\hat{\mathcal{Z}}_{2 s}(n, \rho)\right), \quad(n, s, \rho) \rightarrow \infty .
$$

\subsection{Diagrams $\mathcal{G}^{(c)}(\bar{\nu})$ and their realizations}

Each walk $\mathcal{W}_{2 s}$ generates a set of numerical data, $\bar{\nu}=\left(\nu_{2}, \nu_{3}, \ldots, \nu_{s}\right)$, where $\nu_{k}$ is the number of vertices $\beta_{i}$ of $\bar{g}\left(\mathcal{W}_{2 s}\right)$ such that their self-intersection degree is equal to $k, \varkappa\left(\beta_{i}\right)=k$. To estimate the number of elements of the set $\tilde{\mathbb{W}}_{2 s}$, we construct a kind of diagrams $\mathcal{G}^{(c)}(\bar{\nu})$.

To explain general principles of the estimates, let us start with the construction of noncolored diagram $\mathcal{G}(\bar{\nu})$. This diagram consists of $|\bar{\nu}|=\sum_{k=2}^{s} \nu_{k}$ vertices. We arrange these vertices in $s-1$ levels, the $k$-th level contains $\nu_{k}$ vertices. Each vertex $v$ of $k$-th level is attributed by $k$ half-edges that have heads attached to $v$ but have no tails. Instead of the tail of each edge, we join a square box (or window) to it. Then any vertex $v$ of this $k$-th level has $k$ edge-boxes (or edge-windows) attached.

Given $\mathcal{G}(\bar{\nu})$, one can attribute to its edge-windows the values from the set $\{1,2, \ldots, s\}$ such that there is no pair of windows with the same value. The diagram together with the corresponding values produces a realization of $\mathcal{G}(\bar{\nu})$ that we denote by $\langle\mathcal{G}(\bar{\nu})\rangle_{s}$.

The principal observation used by the Sinai-Soshnikov method is that an even closed walk $\mathcal{W}_{2 s}$ can be completely determined by its values at the marked instant of time added by a family of rules that indicate the values of the walk at the non-marked instant of time. Given Dyck path $\theta_{s}$ and a realization $\langle\mathcal{G}(\bar{\nu})\rangle_{s}$, the positions of the walk at the marked instants of time are completely determined.

The values at the non-marked instant of time are determined by a family of rules $\mathbb{Y}(\bar{\nu})$ that indicate the way to leave a vertex $\beta$ of self-intersection with the help of the non-marked 
step out. It is shown in $[22,23]$ that if $\varkappa(\beta)=k$, then the number of the exit rules at this vertex is bounded as follows, $|\mathbb{Y}(\beta)| \leq(2 k)^{k}$. The rigorous proof of this upper bound is given in [16] (see also [10]). No such rule as $\Upsilon$ is needed for the non-marked instants of time when the walk leaves a vertex of the self-intersection degree 1 because in this case the continuation of the run is uniquely determined. Then the total number of the rules can be estimated as follows,

$$
|\mathbb{Y}(\bar{\nu})| \leq \prod_{k=2}^{s}(2 k)^{k \nu_{k}}
$$

The number of all possible realizations of $\mathcal{G}(\bar{\nu})$ is given by the following expression

$$
\sum_{\langle\mathcal{G}(\bar{\nu})\rangle_{s}} 1=\frac{s !}{\nu_{2} !(2 !)^{\nu_{2}} \nu_{3} !(3 !)^{\nu_{3}} \cdots \nu_{s} !(s !)^{\nu_{s}} \cdot(s-\|\bar{\nu}\|) !},
$$

where $\|\bar{\nu}\|=\sum_{k=2}^{s} k \nu_{k}$. It is easy to see that the following upper bound is true,

$$
\sum_{\langle\mathcal{G}(\bar{\nu})\rangle_{s}} 1 \leq \prod_{k=2}^{s} \frac{1}{\nu_{k} !}\left(\frac{s^{k}}{k !}\right)^{\nu_{k}} .
$$

Combining this inequality with (3.5), we conclude that the number of elements in $\mathbb{W}_{2 s}(\bar{\nu})$ can be estimated as follows,

$$
\left|\mathbb{W}_{2 s}\right| \leq \mathrm{t}_{s} \prod_{k=2}^{s} \frac{1}{\nu_{k} !}\left(\frac{(2 k)^{k} s^{k}}{k !}\right)^{\nu_{k}} \leq \mathrm{t}_{s} \prod_{k=2}^{s} \frac{\left(C_{1} s\right)^{k \nu_{k}}}{\nu_{k} !}, \quad \text { where } \quad C_{1}=\sup _{k \geq 1} \frac{(2 k+2)}{(k !)^{1 /(k+1)}}
$$

We have introduced the constant $C_{1}$ in the form that simplifies further computations.

The upper bound (3.6) clearly explains the role of the diagrams $\mathcal{G}(\bar{\nu})$ in the estimates of the number of walks. However, it is rather rough and does not give inequalities needed in the majority of cases of interest. In particular, the estimate (3.6) is hardly compatible with the upper bound of the weight of walks (3.1) in the case of dilute random matrices.

To improve the upper bound (3.6), we adapt the diagram technique to our model by introducing more informative diagrams based on $\mathcal{G}(\bar{\nu})$. Also, we formulate a new filtering principle to estimate more accurately the number of walks. A kind of the filtration principle has been implicitly used already by Ya. Sinai and A. Soshnikov. The rigorous formulation of the filtration technique is given in [10]. In paper [12] it was adapted to the study of the moments of dilute random matrices.

Let us describe the construction of the color $\operatorname{diagram} \mathcal{G}^{(c)}(\bar{\nu}, \bar{p}, \bar{q})$ determined by parameters $\bar{\nu}=\left(\nu_{2}, \ldots, \nu_{s}\right), \bar{p}=\left(p_{2}, \ldots, p_{s}\right)$ and $\bar{q}=\left(q_{2}, \ldots, q_{s}\right)$. We start with the non-colored diagram $\mathcal{G}(\bar{\nu})$ and consider $\nu_{k}$ vertices of the $k$-th level of it. We fill the second edge-box attached to each vertex by using the set $\{1, \ldots, s\}$. This can be done by

$$
\frac{s !}{\nu_{k} !\left(s-\nu_{k}\right) !} \leq \frac{s^{\nu_{k}}}{\nu_{k} !}=\frac{s^{r_{k}+p_{k}+q_{k}}}{\nu_{k} !}
$$

ways. Then we color the $\nu_{k}$ vertices in blue, red and green colors by one of $\frac{\nu_{k} !}{r_{k} ! p_{k} ! q_{k} !}$ ways, where $r_{k}=\nu_{k}-p_{k}-q_{k}$. Then we color corresponding edge-boxes in grey, blue, red and green 
colors. The base edge-boxes of the first arrivals attached to blue vertices are colored in blue. Instead of boxes, the base edges of the red and green vertices get circles colored with respect to the color of the corresponding vertex.

Taking the empty $k-2$ edge-boxes attached to green or red vertex, we fill them with the values from the set $\{1, \ldots, s\}$. This can be done by not more than $s^{k-2} /(k-2)$ ! ways. Regarding the edges of the first arrivals at red and green vertices that remain empty, we replace corresponding boxes by circles colored according to the color of the vertex.

Let us consider $k-1$ empty edge-boxes attached to a blue vertex and fill them with the values from $\{1, \ldots, s\}$. Ignoring the restriction of the edge-box of the second arrival, we estimate the number of ways to do this by expression $s^{k-1} /(k-1)$ !.

This procedure being performed at each level independently, we get the following estimate from above of the number of different realizations of color diagrams,

$$
\sum_{\left\langle\mathcal{G}^{(c)}(\bar{\nu}, \bar{p}, \bar{q})\right\rangle_{s}} 1 \leq \prod_{k=2}^{s} \frac{1}{r_{k} !}\left(\frac{s^{k}}{(k-1) !}\right)^{r_{k}} \cdot \frac{1}{p_{k} !}\left(\frac{s^{k-1}}{(k-2) !}\right)^{p_{k}} \cdot \frac{1}{q_{k} !}\left(\frac{s^{k-1}}{(k-2) !}\right)^{q_{k}} .
$$

The filtration procedure is follows: we consider a realization of the color diagram $\left\langle\mathcal{G}^{(c)}\right\rangle_{s}$ such that all grey, blue, red and green boxes of edge-windows of $\mathcal{G}^{(c)}$ are filled with different values of $\{1, \ldots, s\}$ while the red and green circles of the first arrivals at the $q$-vertices and $p$-vertices remain empty.

Having a Dyck path $\theta_{s}$ and a rule $\Upsilon \in \mathbb{Y}(\bar{\nu})$ pointed out, we start the run of the walk $\mathcal{W}$ according $\theta_{s},\left\langle\mathcal{G}^{(c)}\right\rangle_{s}$ and $\Upsilon$ till the marked instant of the first $p$-edge or $q$-edge appear. Let us denote by $v^{\prime}$ the corresponding vertex of the diagram $\mathcal{G}^{(c)}$. Let us denote the marked instant mentioned above by $\tau^{\prime}$ with $t^{\prime}=\xi_{\tau^{\prime}}$ and assume that the sub-walk $\mathcal{W}_{\left[0, t^{\prime}-1\right]}$ get its end value $\beta=\mathcal{W}_{\left[0, t^{\prime}-1\right]}\left(t^{\prime}-1\right)$. Then at the instant of time $t^{\prime}$ the walk has to choose one of the admissible vertices from the set $\Gamma=\left\{\gamma_{1}, \ldots, \gamma_{L}\right\}$ such that the edge $\left(\beta, \gamma_{j}\right)$ possesses the properties of either $p$-edge or $q$-edge, respectively. Clearly, the set $\Gamma$ depends on the color of the edge-box with $\tau^{\prime}$. Once the vertex $\gamma_{j}$ is chosen, we take the marked instant of the first arrival at $\gamma_{j}$ and record its value to the edge-box of the first arrival $\mathcal{O}_{1}\left(v^{\prime}\right)$. Clearly, the number of walk is bounded by $|\Gamma|$. This is why it is natural to say that we apply the filtering of all possible values to fill $\mathcal{O}_{1}\left(v^{\prime}\right)$.

Having chosen the value of $\mathcal{O}_{1}$, we continue the run of the walk, if it is possible, till the marked value of the second arrival at the next in turn red or green vertex $v^{\prime \prime}$ is seen. Then the filtering procedure is repeated. When all the walk is constructed, if it exists, we denote by $\left\langle\left\langle\mathcal{G}^{(c)}\right\rangle_{s}^{(b)}\right\rangle_{\mathcal{W}}$ the set of values in red and green circles obtained during this run of $\mathcal{W}$.

Lemma 3.3. Given a realization of a color diagram $\left\langle\mathcal{G}^{(c)}(\bar{\nu}, \bar{p}, \bar{q})\right\rangle_{s}$, let us denote by $\mathbb{W}_{2 s}\left(D,\left\langle\mathcal{G}^{(c)}(\bar{\nu}, \bar{p}, \bar{q})\right\rangle_{s}, \Upsilon\right)$ the set of walks $\mathcal{W}_{2 s}$ that have this realization of $\mathcal{G}^{(c)}$ and follow the rule $\Upsilon$ and such that the maximal exit degree

$$
\mathcal{D}\left(\mathcal{W}_{2 s}\right)=\max _{\beta \in \mathbb{V}\left(\mathcal{W}_{2 s}\right)}|\Delta(\beta)|
$$

is equal to $D, \mathcal{D}\left(\mathcal{W}_{2 s}\right)=D$. Then the number of possible realizations of the values of red and green circles of $\mathcal{G}^{(c)}$ admits the following upper bound,

$$
\left|\left\langle\left\langle\mathcal{G}^{(c)}\right\rangle_{s}\right\rangle_{\mathcal{W}}\right| \leq 2^{|\bar{q}|} D^{|\bar{p}|}
$$


where $|\bar{q}|=\sum_{k=2}^{s} q_{k}$ and $|\bar{p}|=\sum_{k=2}^{s} p_{k}$ and therefore

$$
\left|\mathbb{W}_{2 s}\left(D,\left\langle\mathcal{G}^{(c)}(\bar{\nu}, \bar{p}, \bar{q})\right\rangle_{s}, \Upsilon\right)\right| \leq 2^{|\bar{q}|} D^{|\bar{p}|} \mathrm{t}_{s} .
$$

Proof of Lemma 3.3. Let us prove (3.9) in the case when the color diagram $\mathcal{G}^{(c)}$ has the only one red vertex $v$. Following the filtration principle, we take a Dyck path $\theta_{s}$ and perform the run of the walk in accordance with the data given by the self-intersections of $\left\langle\mathcal{G}^{(c)}(\bar{\nu}, \bar{p}, \bar{q})\right\rangle_{s}^{(b)}$ and $\Upsilon$ till the value $\xi_{\tau^{\prime}}$ appear, where $\tau^{\prime}$ is attributed to the second arrival edge-box attached to $v$. By the definition, the edge $\left(\mathcal{W}\left(\xi_{\tau^{\prime}}-1\right), \mathcal{W}\left(\xi_{\tau^{\prime}}-1\right)\right)=(\beta, \gamma)=e$ is red only in the case when the edge $\tilde{e}=(\gamma, \beta)$ is the edge either of the first or the second arrival at $\gamma$ and $\tilde{e}<e$. Therefore the sub-walk $\mathcal{W}_{\left[0, \xi_{\tau^{\prime}}-1\right]}$ has not more that two vertices available to join at the instant $\xi_{\tau^{\prime}}$. This explains the factor 2 in the left-hand side of (3.9).

In the case when $v$ is the only one green vertex, the sub-walk $\mathcal{W}_{\left[0, \xi_{\tau^{\prime}}-1\right]}$ has the set $\Delta(\beta)$ completely determined, and the vertex to join at the instant $\xi_{\tau^{\prime}}$ necessarily belongs to this set. The upper bound (3.9) in the general case can be proved by recurrence.

Now we can estimate the number of walks that have a color diagram $\mathcal{G}^{(c)}(\bar{\nu}, \bar{p}, \bar{q})$ and the maximal exit degree $D$,

$$
\left|\mathbb{W}_{2 s}\left(D, \mathcal{G}^{(c)}(\bar{\nu}, \bar{p}, \bar{q})\right)\right| \leq \mathrm{t}_{s} \prod_{k=2}^{s} \frac{\left(C_{1} s\right)^{k r_{k}}}{r_{k} !} \cdot \frac{\left(D\left(C_{1} s\right)^{k-1}\right)^{p_{k}}}{p_{k} !} \cdot \frac{\left(2\left(C_{1} s\right)^{k-1}\right)^{q_{k}}}{q_{k} !}
$$

This relation follows from inequalities (3.5), (3.8) and (3.9).

We will use Lemma 3.3 and a version of relation (3.10) in the proof of Theorem 2.1 below. However, to get the estimates we need, we have to show that the number of Catalan trees $\mathcal{T}_{s}$ generated by the elements of $\mathbb{W}_{2 s}\left(D, \mathcal{G}^{(c)}\right)$ is exponentially small with respect to the total number $\mathrm{t}_{s}$ of all $\mathcal{T}_{s}[23,25]$. To do this, we need to study the vertex of maximal exit degree of walks $\mathcal{W}_{2 s}$ in more details.

\subsection{Vertex of maximal exit degree, arrival cells and BTS-instants}

Let us consider a walk $\mathcal{W}_{2 s}$ and find the first letter that we denote by $\breve{\beta}$ such that

$$
|\Delta(\breve{\beta})|=\mathcal{D}\left(\mathcal{W}_{2 s}\right) .
$$

We will refer to $\breve{\beta}$ as to the vertex of maximal exit degree and denote for simplicity $D=\mathcal{D}\left(\mathcal{W}_{2 s}\right)$.

To classify the arrival instants at $\breve{\beta}$, we need to determine reduction procedures similar to those considered in [16] and further modified in [12]. Certain elements of the reduction procedure of [16] were independently introduced in paper [6].

\subsubsection{Reduction procedures and reduced sub-walks}

Given $\mathcal{W}_{2 s}$, let $t^{\prime}$ be the minimal instant of time such that

i) the step $\mathfrak{s}_{t^{\prime}}$ is the marked step of $\mathcal{W}_{2 s}$;

ii) the consecutive to $\mathfrak{s}_{t^{\prime}}$ step $\mathfrak{s}_{t^{\prime}+1}$ is non-marked;

iii) $\mathcal{W}_{2 s}\left(t^{\prime}-1\right)=\mathcal{W}_{2 s}\left(t^{\prime}+1\right)$. 
If such $t^{\prime}$ exists, we apply to the ensemble of steps $\mathfrak{S}=\left\{\mathfrak{s}_{t}, 1 \leq t \leq 2 s, \mathfrak{s}_{t} \in \mathcal{W}_{2 s}\right\}$ a reduction $\dot{\mathcal{R}}$ that removes from $\mathfrak{S}$ two consecutive elements $\mathfrak{s}_{t^{\prime}}$ and $\mathfrak{s}_{t^{\prime}+1}$; we denote $\dot{\mathcal{R}}(\mathfrak{S})=\mathfrak{S}^{\prime}$. The ordering time labels of elements of $\mathfrak{S}^{\prime}$ are inherited from those of $\mathfrak{S}$.

The new sequence $\mathfrak{S}^{\prime}$ can be regarded again as an even closed walk. We can apply to this new walk the reduction procedure $\dot{\mathcal{R}}$. Repeating this operation maximally possible number of times $m$, we get the walk

$$
\dot{\mathcal{W}}_{2 \dot{s}}=(\dot{\mathcal{R}})^{m}\left(\mathcal{W}_{2 s}\right), \quad \dot{s}=s-m,
$$

that we refer to as the strongly reduced walk. We denote $\dot{\mathfrak{S}}=(\dot{\mathcal{R}})^{m}(\mathfrak{S})$ and say that $\dot{\mathcal{R}}$ is the strong reduction procedure.

We introduce a weak reduction procedure $\breve{R}$ of $\mathfrak{S}$ that removes from $\mathfrak{S}_{2 s}$ the pair $\left(\mathfrak{s}_{t^{\prime}}, \mathfrak{s}_{t^{\prime}+1}\right)$ in the case when the conditions (i)-(iii) are verified and

iv) $\mathcal{W}_{2 s}\left(t^{\prime}\right) \neq \breve{\beta}$.

We denote by

$$
\breve{\mathcal{W}}_{2 \breve{s}}=(\breve{\mathcal{R}})^{l}\left(\mathcal{W}_{2 s}\right), \quad \breve{s}=s-l
$$

the result of the action of maximally possible number of consecutive weak reductions $\breve{\mathcal{R}}$ and denote $\breve{\mathfrak{S}}=(\breve{\mathcal{R}})^{l}(\mathfrak{S})$. In what follows, we sometimes omit the subscripts $2 \dot{s}$ and $2 \breve{s}$. Regarding the example walk $\mathcal{W}_{16}(2.3)$, we observe that $\breve{\beta}=\alpha_{3}$ and that the strongly and weakly reduced walks coincide and are as follows,

$$
\dot{\mathcal{W}}_{8}=\breve{\mathcal{W}}_{8}=\left(\alpha_{1}, \alpha_{2}, \alpha_{3}, \alpha_{5}, \alpha_{1}, \alpha_{2}, \alpha_{3}, \alpha_{5}, \alpha_{1}\right)
$$

Taking the difference $\breve{\mathfrak{S}} \backslash \dot{\mathfrak{S}}=\ddot{\mathfrak{S}}$, we see that it represents a collection of sub-walks, $\ddot{W}=\cup_{j} \ddot{\mathcal{W}}^{(j)}$. Each sub-walk $\ddot{\mathcal{W}}^{(j)}$ can be reduced by a sequence of the strong reduction procedures $\dot{\mathcal{R}}$ to an empty walk. We say that $\ddot{\mathcal{W}}^{(j)}$ is of the Dyck-type structure. It is easy to see that any $\ddot{W}^{(j)}$ starts by a marked step and ends by a non-marked steps and there is no steps of $\dot{W}$ between these two steps of $\ddot{\mathcal{W}}^{(j)}$. We say that $\ddot{\mathcal{W}}^{(j)}$ is the non-split sub-walk.

It is not hard to see that the collection of steps $\breve{S}=\mathfrak{S} \backslash \breve{\mathfrak{S}}$ is given by a collection of subsets $\breve{\mathfrak{S}}=\cup_{k} \check{\mathfrak{S}}^{(k)}$, each of $\check{\mathfrak{S}}^{(k)}$ represents a non-split Dyck-type sub-walk $\check{\mathcal{W}}^{(k)}$,

$$
\check{W}=\cup_{k} \check{\mathcal{W}}^{(k)} .
$$

In this definition, we assume that each sub-walk $\breve{\mathcal{W}}^{(k)}$ is maximal by its length.

\subsubsection{Arrival instants and Dyck-type sub-walks attached to $\breve{\beta}$}

Given $\mathcal{W}_{2 s}$, let us consider the instants of time $0 \leq t_{1}<t_{2}<\ldots t_{R} \leq 2 s$ such that for all $i=1, \ldots, R$ the walk arrives at $\breve{\beta}$ by the steps of $\breve{\mathcal{W}}_{2 \breve{s}}$,

$$
\mathcal{W}_{2 s}\left(t_{i}\right)=\breve{\beta} \quad \text { and } \quad \mathfrak{s}_{t_{i}} \in \breve{\mathcal{W}}_{2 \breve{s}}, \quad i=1,2, \ldots, R \text {. }
$$

We say that $t_{i}$ are the $\breve{t}$-arrival instants of time of $\mathcal{W}_{2 s}$. Let us consider a sub-walk that corresponds to the subset $\mathfrak{S}_{\left[t_{i}+1, t_{i+1}\right]}=\left\{\mathfrak{s}_{t}, t_{i}+1 \leq t \leq t_{i+1}\right\} \subseteq \mathfrak{S}$; we denote this sub-walk by $\mathcal{W}_{\left[t_{i}, t_{i+1}\right]}$. In general, we denote a sub-walk that is not necessary even and/or closed by $\mathcal{W}_{\left[t^{\prime}, t^{\prime \prime}\right]}$ also. 
Let us consider the interval of time $\left[t_{i}+1, t_{i+1}-1\right]$ between two consecutive $\breve{t}$-arrivals at $\breve{\beta}$. It can happen that $\mathcal{W}_{2 s}$ arrives at $\breve{\beta}$ at some instants of time $t^{\prime} \in\left[t_{i}+1, t_{i+1}-1\right], \mathcal{W}_{2 s}\left(t^{\prime}\right)=\breve{\beta}$. We denote by $\check{t}_{(i)}$ the maximal value of such $t^{\prime}$.

Lemma 3.4. The sub-walk $\mathcal{W}_{\left[t_{i}, \breve{t}_{(i)}\right]}$ coincides with one of the maximal Dyck-type sub-walks $\check{\mathcal{W}}^{\left(k^{\prime}\right)}$ of (3.13).

Lemma 3.4 is proved in [12].

Let us consider a collection of all marked exit edges from $\breve{\beta}$ performed by the marked steps on the interval of time $\left[t_{i}, \tilde{t}_{(i)}\right]$ and denote this collection by $\check{\Delta}_{i}$. We say that $\check{\Delta}_{j}$ represents the exit sub-clusters of Dyck type attached to $\breve{\beta}$. or simply that $\check{\Delta}_{j}$ are the exit sub-clusters of $\mathcal{W}_{2 s}$. We denote their cardinalities by $d_{j}=\left|\breve{\Delta}_{j}\right|$. The exit sub-clusters are ordered in natural way. To keep a unified description, we accept the existence of empty exit sub-clusters; then we get equality $D=\sum_{j=1}^{R} d_{j}, d_{j} \geq 0$. Clearly, any exit sub-cluster is attributed to a uniquely determined $\breve{t}$-arrival instant at $\breve{\beta}$.

Regarding the reduced walk $\breve{\mathcal{W}}_{2 \breve{s}}$ of $\mathcal{W}_{2 s}$ (3.12), we can determine corresponding Dyck path $\breve{\theta}_{2 \breve{s}}=\theta\left(\breve{\mathcal{W}}_{2 \breve{s}}\right)$ and the tree $\breve{\mathcal{T}} \breve{s}_{\breve{s}}=\mathcal{T}(\breve{\theta})$. It is easy to show that $\breve{\mathcal{T}}_{\breve{s}}$ is a sub-tree of the original tree $\mathcal{T}_{s}=\mathcal{T}\left(\theta\left(\mathcal{W}_{2 s}\right)\right)$. One can introduce the difference $\check{\mathcal{T}}=\mathcal{T}_{s} \backslash \breve{\mathcal{T}_{\breve{s}}}$ and say that it is represented by a collection of sub-trees $\check{\mathcal{T}}^{(j)}$.

Returning to the Catalan tree $\mathcal{T}\left(\theta_{2 s}\right)$, let us consider the chronological run over it that we denote by $\mathfrak{R}_{\mathcal{T}}$. Then the $\breve{t}$-arrival instant $t_{l}$ (3.14) determines the step $\varpi_{l}$ of $\mathfrak{R}_{\mathcal{T}}$. Also the corresponding vertex $\check{v}_{l}$ of the tree $\mathcal{T}_{s}$ is determined. It is clear that $\check{v}_{l}$ are not necessarily different for different $l$.

The sub-trees $\check{\mathcal{T}}^{(l)}$ are attached to $\check{v}_{l}$ and the chronological run over $\check{\mathcal{T}}^{(l)}$ starts immediately after the step $\varpi_{l}$ is performed. We will say that these steps $\varpi_{l}, 1 \leq l \leq R$ represent the nest cells from where the sub-trees $\check{\mathcal{T}}^{(l)}, 1 \leq l \leq L$ grow. It is clear that the sub-tree $\mathcal{T}_{l}$ has $d_{l} \geq 0$ edges attached to its root $\varrho_{l}$ and this root coincides with the vertex $\check{v}_{l}$. Returning to $\mathcal{W}_{2 s}$, we will say that the arrival instants of time $\breve{t}_{l}$ represent the arrival cells at $\breve{\beta}$. In the next sub-section, we describe a classification of the arrival cells at $\breve{\beta}$ that represents a natural improvement of the approach proposed in [16].

\subsubsection{Classification of arrival cells at $\breve{\beta}$}

Let us consider a walk $\mathcal{W}_{2 s}$ together with its reduces counterparts $\dot{\mathcal{W}}_{2 \dot{s}}=\dot{\mathcal{W}}$ and $\breve{\mathcal{W}}_{2 \breve{s}}=\breve{\mathcal{W}}$. Let $t_{i}$ denote a $\breve{t}$-arrival cell (3.4). If the step $\mathfrak{s}_{t_{i}}$ of $\mathcal{W}_{2 s}$ is marked, then we say that $t_{i}$ represents a proper cell at $\breve{\beta}$. If the step $\mathfrak{s}_{t_{i}}$ is non-marked and $\mathfrak{s}_{t_{i}} \in \ddot{\mathcal{W}}=\breve{\mathcal{W}} \backslash \dot{\mathcal{W}}$, then we say that $t_{i}$ represents a mirror cell at $\breve{\beta}$. If the step $\mathfrak{s}_{t_{i}} \in \mathcal{W}$ is non-marked, then we say that $t_{i}$ represents an imported cell at $\breve{\beta}$.

Let us consider $I$ proper cells $\ddot{t}_{i}$ such that $\mathfrak{s}_{\ddot{t}_{i}}$ belongs to $\ddot{\mathfrak{S}}$. We denote by $x_{i}$ the corresponding marked instants, $x_{i}=\xi_{\ddot{t}_{i}}, 1 \leq i \leq I$ and write that $\bar{x}_{I}=\left(x_{1}, \ldots, x_{I}\right)$. It is easy to see that each proper cell $x_{i}$ can be attributed by a number 1 or 0 in dependence of whether it produces a corresponding mirror cell at $\breve{\beta}$ or not. We denote this number by $m_{i} \in\{0,1\}$ and write that $M=\sum_{i=1}^{I} m_{i}$ and $\bar{m}_{I}=\left(m_{1}, \ldots, m_{I}\right)$. Clearly, $M \leq I$.

Regarding the strongly reduced walk $\dot{\mathcal{W}}_{2 \dot{s}}$, we denote by $\dot{t}_{k}$ the proper cells such that the steps $\mathfrak{s}_{\dot{t}_{k}} \in \dot{\mathfrak{S}}$. Corresponding to $\dot{t}_{k}$ marked instants will be denoted by $z_{k}, 1 \leq k \leq K$. Then $\bar{z}_{K}=\left(z_{1}, \ldots, z_{K}\right)$ and clearly $\varkappa(\breve{\beta})=I+K$. 
Given $\mathcal{W}_{2 s}$ with non-empty set $\dot{\mathfrak{S}}$, there exists at least one pair of elements of $\dot{\mathfrak{S}}$ denoted by $\left(\mathfrak{s}^{\prime}, \mathfrak{s}^{\prime \prime}\right)$ such that $\mathfrak{s}^{\prime}$ is a marked step of $\dot{\mathcal{W}}_{2 \dot{s}}, \mathfrak{s}^{\prime \prime}$ is the non-marked one and $\mathfrak{s}^{\prime \prime}$ follows immediately after $\mathfrak{s}^{\prime}$ in $\dot{\mathfrak{S}}$. We refer to each pair of this kind as to the pair of broken tree structure steps of $\mathcal{W}_{2 s}$ or in abbreviated form, the BTS-pair of $\mathcal{W}_{2 s}$. If $\tau^{\prime}$ is the marked instant that corresponds to $\mathfrak{s}^{\prime}$, we will simply say that $\tau^{\prime}$ is the BTS-instant of $\mathcal{W}_{2 s}$ [16].

Regarding the strongly reduced walk $\dot{\mathcal{W}}$, let us consider a non-marked arrival step at $\breve{\beta}$ that we denote by $\overline{\mathfrak{s}}=\mathfrak{s}_{\bar{t}}$. Then one can find a uniquely determined marked instant $\tau^{\prime}$ such that all steps $\mathfrak{s}_{t} \in \dot{\mathfrak{S}}$ with $\xi_{\tau^{\prime}}+1 \leq t \leq \bar{t}$ are the non-marked ones. Let us denote by $t^{\prime \prime}$ the instant of time of the first non-marked step $\mathfrak{s}_{\bar{t}^{\prime \prime}} \in \hat{\mathfrak{S}}$ of this series of non-marked steps. Then $\left(\mathfrak{s}_{t^{\prime}}, \mathfrak{s}_{t^{\prime \prime}}\right)$ with $t^{\prime}=\xi_{\tau^{\prime}}$ is the BTS-pair of $\mathcal{W}_{2 s}$ that corresponds to $\bar{t}$. We will say that $\bar{t}$ is attributed to the corresponding BTS-instant $\tau^{\prime}$. It can happen that several arrival instants $\bar{t}_{i}$ are attributed to the same BTS-instant $\tau^{\prime}$. We will also say that the BTS-instant $\tau^{\prime}$ generates the imported cells that are attributed to it.

Let us consider a BTS-instant $\tau$ such that $\mathcal{W}_{2 s}\left(\xi_{\tau}\right)=\breve{\beta}$. As it is said above, we denote such marked instants by $z_{k}, 1 \leq k \leq K$. Assuming that a marked BTS-instant $z_{k}$ generates $f_{k}^{\prime} \geq 0$ imported cells, we denote by $\varphi_{1}^{(k)}, \ldots, \varphi_{f_{k}^{\prime}}^{(k)}$ the positive numbers such that

$$
\mathcal{W}_{2 s}\left(\xi_{z_{k}}+\sum_{j=1}^{l} \varphi_{j}^{(k)}\right)=\breve{\beta} \quad \text { for all } \quad 1 \leq l \leq f_{k}^{\prime} .
$$

If for some $\tilde{k}$ we have $f_{\tilde{k}}^{\prime}=0$, then we will say that $z_{\tilde{k}}$ does not generate any imported cell at $\breve{\beta}$. We denote $\bar{\varphi}^{(k)}=\left(\varphi_{1}^{(k)}, \ldots, \varphi_{f_{k}^{\prime}}^{(k)}\right)$.

Let us consider a BTS-instant $\tau$ that generates imported cells at $\breve{\beta}$ and such that $\mathcal{W}_{2 s}\left(\xi_{\tau}\right) \neq$ $\breve{\beta}$. We denote these BTS-instants by $y_{j}, 1 \leq j \leq J$. Assuming that a marked BTS-instant $y_{j}$ generates $f_{j}^{\prime \prime}+1$ imported cells, $f_{j}^{\prime \prime} \geq 0$, we denote by $\Lambda_{j}, \psi_{1}^{(j)}, \ldots, \psi_{f_{j}^{\prime \prime}}^{(j)}$ the positive numbers such that $\mathcal{W}_{2 s}\left(\xi_{y_{j}}+\Lambda_{j}\right)=\breve{\beta}$ and

$$
\mathcal{W}_{2 s}\left(\xi_{y_{j}}+\Lambda_{j}+\sum_{l=1}^{k} \psi_{l}^{(j)}\right)=\breve{\beta} \quad \text { for all } \quad 1 \leq k \leq f_{j}^{\prime \prime} .
$$

In this case we will say that the first arrival at $\breve{\beta}$ given by the instant of time $\xi_{y_{j}}+\Lambda_{j}$ represents the principal imported cell at $\breve{\beta}$. All subsequent arrivals at $\breve{\beta}$ given by (3.13) represent the secondary imported cells at $\breve{\beta}$. We will say that $y_{j}$ is the remote BTS-instant with respect to $\breve{\beta}$ and will use denotations $\bar{y}_{J}=\left(y_{1}, \ldots, y_{J}\right)$ and $\bar{\Lambda}_{J}=\left(\Lambda_{1}, \ldots, \Lambda_{J}\right)$. We also denote $\bar{\psi}^{(j)}=\left(\psi_{1}^{(j)}, \ldots, \psi_{f_{j}^{\prime \prime}}^{(j)}\right)$.

We see that for a given walk $\mathcal{W}_{2 s}$, the proper, mirror and imported cells at its vertex of maximal exit degree are characterized by the set of parameters, $(\bar{x}, \bar{m})_{I},\left(\bar{z}, \Phi, \bar{f}^{\prime}\right)_{K}$, where $\Phi_{K}=\left(\bar{\varphi}^{(1)}, \ldots \bar{\varphi}^{(K)}\right), \bar{f}_{K}^{\prime}=\left(f_{1}^{\prime}, \ldots, f_{K}^{\prime}\right)$ and $\left(\bar{y}, \bar{\Lambda}, \Psi, \bar{f}^{\prime \prime}\right)_{J}$, where $\Psi_{J}=\left(\bar{\psi}^{(1)}, \ldots, \bar{\psi}^{(J)}\right)$, $\bar{f}_{J}^{\prime \prime}=\left(f_{1}^{\prime \prime}, \ldots, f_{J}^{\prime \prime}\right)$. We also denote

$$
F^{\prime}=\sum_{k=1}^{K} f_{k}^{\prime} \quad \text { and } \quad F^{\prime \prime}=\sum_{j=1}^{J} f_{j}^{\prime \prime} .
$$


Summing up, we observe that the vertex $\breve{\beta}$ with $\varkappa(\breve{\beta})=I+K$ has the total number of cells given by $R=I+M+K+J+F$, where $F=F^{\prime}+F^{\prime \prime}$. In what follows, we denote the family of the parameters described above by

$$
\mathcal{P}_{R}=\left\{(\bar{x}, \bar{m})_{I},\left(\bar{y}, \bar{\Lambda}, \Psi, \bar{f}^{\prime \prime}\right)_{J},\left(\bar{z}, \Phi, \bar{f}^{\prime}\right)_{K}\right\} .
$$

\subsection{Proof of Theorem 3.1}

We are going to estimate the number of walks in a family of walks $\tilde{\mathbb{W}}_{2 s}(D)$ that have a vertex of maximal exit degree $D$. We rewrite (3.2) in the following from

$$
\tilde{\mathcal{Z}}_{2 s}(n, \rho)=\sum_{D=1}^{s} \sum_{\mathcal{W}_{2 s} \in \tilde{\mathbb{W}}_{2 s}(D)} \Pi_{a}\left(\mathcal{W}_{2 s}\right) \Pi_{b}\left(\mathcal{W}_{2 s}\right) \cdot\left|\mathcal{C}_{\mathcal{W}_{2 s}}\right|,
$$

where $\left|\mathcal{C}_{\mathcal{W}_{2 s}}\right|$ is given by (2.4). To estimate the number of elements in $\tilde{\mathbb{W}}_{2 s}(D)$, we have to consider a kind of color diagrams that have a separate vertex $\breve{v}$ attributed by the parameters from the family $\mathcal{P}_{R}$, namely by $\bar{x}_{I}$ and $\bar{z}_{K}$. Also we have to include into the color diagrams the parameters $\bar{y}_{J}$. Let us describe this new type of color diagrams.

\subsubsection{Color diagrams with a vertex of maximal exit degree}

Let us consider a vertex $\breve{v}$ and attach to it $I+K$ edge-boxes. We denote by $\left\langle\breve{v}_{I, K}\right\rangle_{s}$ a realization of the values of marked instants that fill these boxes. Given $\bar{\nu}, \bar{p}$ and $\bar{q}$, we consider a realization of the corresponding color diagram $\left\langle\mathcal{G}^{(c)}(\bar{\nu}, \bar{p}, \bar{q})\right\rangle_{s}$ and point out $J$ edge-boxes that will provide the marked instants $\bar{y}$. Joining such a realization with chosen $J$ edge-boxes $\left\langle\mathcal{G}_{\bar{y}}^{(c)}(\bar{\nu}, \bar{p}, \bar{q})\right\rangle_{s}^{(b)}$ with $\left\langle\breve{v}_{I, K}\right\rangle_{s}$, we get a realizations of the diagram we need,

$$
\left\langle\breve{\mathcal{G}}_{\bar{x}, \bar{z}, \bar{y}}^{(c)}(\bar{\nu}, \bar{p}, \bar{q})\right\rangle_{s}^{(b)}=\left\langle\breve{v}_{I, K}\right\rangle_{s} \uplus\left\langle\mathcal{G}_{J}^{(c)}(\bar{\nu}, \bar{p}, \bar{q})\right\rangle_{s}^{(b)}=\left\langle\breve{v} \uplus \mathcal{G}^{(c)}\right\rangle_{s}^{(b)} .
$$

The last equality of the formula presented above introduces a denotation for a realization of the diagram we consider.

The number of different realizations of the color diagram $\mathcal{G}^{(c)}(\bar{\nu}, \bar{p}, \bar{q})$ is estimated by the right-hand side of (3.8). Regarding realizations $\left\langle\breve{v}_{I, K}\right\rangle_{s}$, we can write that

$$
\left|\left\langle\breve{v}_{I, K}\right\rangle_{s}\right| \leq \frac{s^{I+K}}{(I+K) !} 2^{I+K}
$$

where the last factor gives the upper bound for the choice of $K$ elements among $I+K$ ones to be marked as the values of $\bar{z}_{K}$. The vertex $\breve{\beta}$ of the walk can be attributed by the weight

$$
\Pi_{a}(\breve{\beta}) \Pi_{b}(\breve{\beta})= \begin{cases}\frac{V_{2}}{n}, & \text { if } \varkappa(\breve{\beta})=1, \\ \frac{1}{n^{2} \rho^{I+K-2}} V_{2}^{2} U^{2(I+K)-4}, & \text { if } \breve{\beta} \text { is an } r \text {-vertex, } \\ \frac{1}{n \rho^{I+K-1}} V_{2} U^{2(I+K)-2}, & \text { if } \breve{\beta} \text { is a } p \text {-vertex or a } q \text {-vertex. }\end{cases}
$$

In the first and in the third cases of (3.19), at least one blue $r$-vertex is necessarily present in $\mathcal{G}^{(c)}(\bar{\nu}, \bar{p}, \bar{q})$. 
Regarding $\left\langle\mathcal{G}^{(c)}(\bar{\nu}, \bar{p}, \bar{q})\right\rangle_{s}$, one can choose $J$ edge-boxes to be labeled as the values of the realization $\langle\bar{y}\rangle$ among $\sum_{k=2}^{s}(k-1) \nu_{k}=\|\bar{\nu}\|_{1}$ edges only. This is because the first arrival to a vertex cannot be the marked BTS-instant. The number of ways to choose $J$ ordered places among $\|\bar{\nu}\|_{1}$ ordered edges can be estimated as follows,

$$
\left(\begin{array}{c}
\|\bar{\nu}\|_{1} \\
J
\end{array}\right) \leq \frac{\left(\|\bar{\nu}\|_{1}\right)^{J}}{J !} \leq \frac{1}{h_{0}^{J}} \exp \left\{h_{0}\|\bar{\nu}\|_{1}\right\}
$$

where $h_{0}>1$ is a constant.

\subsubsection{Exit sub-clusters and cells at $\breve{\beta}$}

The maximal exit degree of a walk $\mathcal{W}_{2 s} \in \mathbb{W}_{2 s}(D)$ can be represented as follows, $D=\dot{D}+$ $\ddot{D}+\check{D}$, where $\dot{D}$ is the number of marked edges of the form $(\breve{\beta}, \gamma)$ that belong to the strongly reduced walk $\dot{\mathcal{W}}(3.12), \ddot{D}$ represents the exit edges that belong to $\ddot{\mathcal{W}}=\breve{\mathcal{W}} \backslash \dot{\mathcal{W}}$. It is known that $\dot{D}=F+J$ and that $F \leq K[16]$ (see also Lemma 5.1 of [10]). Also we observe that $\ddot{D}=M$. Taking into account that $M \leq I$, we can write that

$$
\check{D}=D-M-F-J \geq D-I-K-J .
$$

The remaining $\check{D}$ edges of $\overline{\mathbb{E}}\left(\mathcal{W}_{2 s}\right)$ belong to the exit sub-clusters of the Dyck-type subwalks $\breve{W}^{(k)}$ (3.13) attached to $\breve{\beta}$. They are distributed among $R$ arrival cells at $\breve{\beta}$. We denote by $\bar{d}=\left(\check{d}_{1}, \ldots, \breve{d}_{R}\right)$ a particular distribution such that $\sum_{l=1}^{R} \check{d}_{l}=\check{D}$.

The number of cells $R$ depends on $\left\langle\mathcal{G}^{(c)}\right\rangle_{s}^{(b)}, \theta_{s}$ and $\Upsilon$. However, the inequalities used to get (3.21) show that

$$
R=I+K+M+F+J \leq 2 I+2 K+J=R^{*} .
$$

Then

$$
\sum_{\bar{d}_{R},\left|\bar{d}_{R}\right|=\check{D}} 1=\left(\begin{array}{c}
\check{D}+R-1 \\
R-1
\end{array}\right) \leq\left(\begin{array}{c}
D+R^{*}-1 \\
R^{*}-1
\end{array}\right) .
$$

Elementary analysis shows that if $D \geq 2$, then

$$
\left(\begin{array}{c}
D+R^{*}-1 \\
R^{*}-1
\end{array}\right) \leq h_{0}^{R^{*}} \sup _{R^{*} \geq 2} \frac{1}{h_{0}^{R^{*}-1}}\left(\begin{array}{c}
D+R^{*}-1 \\
R^{*}-1
\end{array}\right) \leq h_{0}^{2 I+2 K+J} \exp \left\{\frac{e D}{h_{0}}\right\}, h_{0}>e .
$$

Indeed, using the well-known estimates

$$
\sqrt{2 \pi n}\left(\frac{n}{e}\right)^{n} \leq n ! \leq e \sqrt{n}\left(\frac{n}{e}\right)^{n}, \quad n \geq 1,
$$

we can write that

$$
\frac{1}{h_{0}^{m}} \frac{(D+m) !}{D ! m !} \leq \frac{1}{h_{0}^{m}} \frac{e}{2 \pi} \sqrt{\frac{D+m}{m D}}\left(1+\frac{m}{D}\right)^{D}\left(1+\frac{D}{m}\right)^{m} \leq \frac{e^{m}}{h_{0}^{m}}\left(1+\frac{D}{m}\right)^{m},
$$

where we take into account that $D+m \leq 2 m D$. Then the last relation of (3.23) follows. Now we are ready to perform the estimates that prove Theorem 3.1. 


\subsubsection{Exponential estimates and $\tilde{\mathcal{Z}}_{2 s}$}

In this subsection we estimate the contribution of the non-tree type walks $\tilde{\mathcal{Z}}_{2 s}$ and prove relation (3.3) with the help of computations that are very similar to those used in the pioneering papers by Ya. Sinai and A. Soshnikov. The following statement can be regarded as the principal result in the rigorous formulation of the method.

Lemma 3.4. Given D, a realization of the color diagram $\left\langle\breve{v} \uplus \mathcal{G}^{(c)}\right\rangle_{s}^{(b)}$ (3.18) and a rule $\Upsilon$, let us consider a family of walks $\mathbb{W}_{2 s}\left(D,\left\langle\breve{v} \uplus \mathcal{G}^{(c)}\right\rangle_{s}, \Upsilon\right)$ such that the vertex of the maximal exit degree $\breve{\beta}$ given by $\breve{v}$ has $D$ exit edges of the form $\left(\breve{\beta}, \gamma_{i}\right)$. Then

$$
\left|\mathbb{W}_{2 s}\left(D,\left\langle\breve{v} \uplus \mathcal{G}^{(c)}\right\rangle_{s}, \Upsilon\right)\right| \leq 2^{|\bar{q}|} D^{|\bar{p}|}\left(e^{\eta} h_{0}^{2}\right)^{I+K+J} e^{-\eta D+e D / h_{0}} \mathrm{t}_{s},
$$

where $\eta=\ln (4 / 3)$.

We prove Lemma 3.4 in Section 5. The walks we consider are of the non-tree type and therefore contain at least one blue $r$-vertex $v_{0}$. Let us divide the sum $\tilde{\mathcal{Z}}_{2 s}$ in two parts in dependence whether $v_{0}=\breve{v}$ or $v_{0} \neq \breve{v}$,

$$
\tilde{\mathcal{Z}}_{2 s}(n, \rho)=\tilde{\mathcal{Z}}_{2 s}^{(1)}+\tilde{\mathcal{Z}}_{2 s}^{(2)},
$$

respectively. Then we can write that

$$
\begin{gathered}
\tilde{\mathcal{Z}}_{2 s}^{(1)}=\sum_{D=1}^{s} \sum_{J=0}^{s}\left(\prod_{k=2}^{s} \sum_{r_{k}, p_{k}, q_{k}}\right)^{\star} \sum_{I, K: I+K \geq 1}\left|\mathcal{C}_{W_{2 s}}\right| \\
\times \sum_{\breve{v} \uplus \mathcal{G}_{s}^{(c)}} \sum_{\left\langle\breve{v} \uplus \mathcal{G}^{(c)}\right\rangle_{s}} \sum_{\mathcal{W}_{2 s} \in \mathbb{W}_{2 s}\left(D,\left\langle\breve{v} \uplus \mathcal{G}^{(c)}\right\rangle_{s}\right)} \Pi_{a, b}\left(\mathcal{W}_{2 s}\right),
\end{gathered}
$$

where the star means that the values of $r_{k}, p_{k}$ and $q_{k}$ are such that $\sum(k-1) \nu_{k} \geq J$ and $\sum r_{k} \geq 1$. The first sum of the second line of (3.26) takes into account the choice of the $J$ places in $\mathcal{G}^{(c)}$ to be marked as the edge-boxes of values $y_{j}$ (see also (3.20)); the second sum is performed over all possible realizations of the diagram $\breve{v} \uplus \mathcal{G}^{(c)}$ with the help of the values from $\{1, \ldots, s\}$ (see $(3.8)$ and $(3.18)$ ).

Using relations (3.1), (3.10), (3.18) and (3.19), we deduce from (3.26) the following upper bound,

$$
\begin{gathered}
\tilde{\mathcal{Z}}_{2 s}^{(1)} \leq \sum_{D=1}^{s} \sum_{J=0}^{s} \sum_{I+K \geq 1} \frac{(2 s)^{I+K}}{(I+K) !}(2(I+K))^{I+K} \cdot h_{0}^{2(I+K)+J} e^{\eta(I+J+K)} \\
\times\left(\prod_{k=2}^{s} \sum_{r_{k}, p_{k}, q_{k}}\right)^{\star} e^{h_{0}\|\bar{\nu}\|_{1}} \frac{1}{r_{k} !}\left(\frac{(2 k)^{k} s^{k}}{(k-1) !}\right)^{r_{k}} \cdot \frac{1}{p_{k} !}\left(\frac{(2 k)^{k} D s^{k-1}}{(k-2) !}\right)^{p_{k}} \cdot \frac{1}{q_{k} !}\left(\frac{(2 k)^{k} 2 s^{k-1}}{(k-2) !}\right)^{q_{k}} \\
\times \frac{V_{2} U^{2(I+K-1)}}{n \rho^{I+K-1}} \cdot\left(\frac{V_{2}^{2}}{n^{2}}\right)^{r_{k}}\left(\frac{V_{2} U^{2}}{n \rho}\right)^{p_{k}+q_{k}}\left(\frac{U^{2}}{\rho}\right)^{(k-2) \nu_{k}}\left(\frac{V_{2}}{n}\right)^{s-\|\bar{\nu}\|-(I+K)}
\end{gathered}
$$

where we denoted $\|\bar{\nu}\|=\sum_{k=2}^{s} k \nu_{k}$. 
Let us consider a constant (cf. (3.6))

$$
C_{2}=\max \left\{\sup _{k \geq 2} \frac{2 k}{((k-1) !)^{1 / k}}, \sup _{k \geq 2} \frac{(2 k)^{k /(k-1)}}{((k-2) !)^{1 /(k-1)}},\right\}
$$

and denote

$$
B=C_{2} h_{0} e^{h_{0}+\eta}=4 C_{2} h_{0} e^{h_{0}} / 3,
$$

where $h_{0}>e$ will be determined below. Remembering that $s=\chi \rho$, we can deduce from (3.27) the following inequality,

$$
\begin{gathered}
\tilde{Z}_{2 s}^{(1)} \leq V_{2}^{s} \sum_{D=1}^{s} e^{-\eta D+e D / h_{0}} n \mathrm{t}_{s} \sum_{J=0}^{s} \sum_{I+K \geq 1} 2 s B\left(2 B \hat{U}^{2} \chi\right)^{I+K-1} \\
\times\left(\prod_{k=2}^{s} \sum_{r_{k}, p_{k}, q_{k}}\right)^{\star} \frac{1}{r_{k} !}\left(\frac{B \hat{U}^{2} s^{2}}{n}\left(B \hat{U}^{2} \chi\right)^{k-2}\right)^{r_{k}} \cdot \frac{1}{p_{k} !}\left(D\left(B \hat{U}^{2} \chi\right)^{k-1}\right)^{p_{k}} \cdot \frac{1}{q_{k} !}\left(2\left(B \hat{U}^{2} \chi\right)^{k-1}\right)^{q_{k}} .
\end{gathered}
$$

If $\chi$ is such that

$$
2 B \hat{U}^{2} \chi \leq 1
$$

then (3.29) implies inequality

$$
\tilde{Z}_{2 s}^{(1)} \leq 4 B s^{3}\left(\exp \left\{\frac{2 B s^{2}}{n}\right\}-1\right) \cdot e^{4 B \hat{U}^{2} \chi} n \mathrm{t}_{s} V_{2}^{s} \sum_{D=1}^{\infty} \exp \left\{-\eta+2 B \hat{U}^{2} \chi+e / h_{0} D\right\} .
$$

Remembering that $\eta=\ln (4 / 3)>0.28$, we see that if

$$
\frac{3 C_{2} U^{2} h_{0} e^{h_{0}}}{V_{2}} \chi+\frac{e}{h_{0}} \leq 0.28
$$

then (3.30) implies relation

$$
\tilde{Z}_{2 s}^{(1)}=o\left(n \mathrm{t}_{s} V_{2}^{s}\right), \quad n, \rho_{n} \rightarrow \infty
$$

provided $\rho_{n}=o\left(n^{1 / 5}\right)(1.6)$. Clearly, the choice of $h_{0}$ and $\chi$ such that

$$
h_{0}=4 e \quad \text { and } \quad \chi \leq \frac{V_{2}}{400 e^{4 e+1} C_{2} U^{2}}
$$

makes (3.29) and (3.31) valid. Let us note that more detailed analysis of the walks with maximal exit degree $D$ show that the factor $s^{3}$ in the right-hand side of (3.30) can be eliminated. However, in the present paper we do not aim the maximal rate of $\rho_{n}$ and therefore the upper bound (3.30) is sufficient for our purposes.

Let us consider the second term of (3.25). The sub-sum $\tilde{\mathcal{Z}}_{2 s}^{(2)}$ can be estimated from above by the expression given by the right-hand side of (3.27), where the sum over $I, K$ is performed over the range $I+K \geq 2$ and the weight factor $V_{2} U^{2(I+K-1)} /\left(n \rho^{I+K-1}\right)$ is replaced by $V_{2}^{2} U^{2(I+K-2)} /\left(n^{2} \rho^{I+K-2}\right)$ (see relation (3.20)) and where the condition $\sum_{k} r_{k} \geq 1$ is omitted. 
Then we can write that

$$
\begin{gathered}
\tilde{Z}_{2 s}^{(2)} \leq n \mathrm{t}_{s} V_{2}^{s} \sum_{D=1}^{s} e^{-\eta D+e D / h_{0}} \sum_{J=0}^{s} \sum_{I+K \geq 2} \frac{2 s^{2} B}{n}\left(2 B \hat{u}^{2} \chi\right)^{I+K-2} \\
\times \prod_{k=2}^{s} \sum_{r_{k}, p_{k}, q_{k}} \frac{1}{r_{k} !}\left(\frac{B \hat{U}^{2} s^{2}}{n}\left(B \hat{U}^{2} \chi\right)^{k-2}\right)^{r_{k}} \cdot \frac{1}{p_{k} !}\left(D\left(B \hat{U}^{2} \chi\right)^{k-1}\right)^{p_{k}} \cdot \frac{1}{q_{k} !}\left(2\left(B \hat{U}^{2} \chi\right)^{k-1}\right)^{q_{k}} .
\end{gathered}
$$

If (3.32) is true, then (3.33) implies the upper bound

$$
\tilde{Z}_{2 s}^{(2)} \leq n \mathrm{t}_{s} V_{2}^{s} \frac{4 s^{4} B}{n} \cdot e^{2 B \hat{U}^{2} \chi} \sum_{D=1}^{\infty} \exp \left\{-\eta+2 B \hat{U}^{2} \chi+e / h_{0} D\right\} .
$$

Then

$$
\tilde{Z}_{2 s}^{(2)}=o\left(n \mathrm{t}_{s} V_{2}^{s}\right)
$$

under conditions of Theorem 2.1. Theorem 3.1 is proved.

\section{Tree-type walks and $\left(2,4^{\star}\right)$-walks}

Let us consider the family $\hat{\mathbb{W}}_{2 s}$ of tree-type walks and separate it into two non-intersecting subsets,

$$
\hat{\mathbb{W}}_{2 s}=\dot{\mathbb{W}}_{2 s} \sqcup \ddot{\mathbb{W}}_{2 s},
$$

where $\dot{\mathbb{W}}_{2 s}$ contains the walks $\mathcal{W}_{2 s}$ such that their weights have the factors $V_{2}=1$ and $V_{4}$ only and the graph $\bar{g}\left(W_{2 s}\right)$ is such that the $V_{4}$-edges do not share a vertex in common. We also denote this set by $\mathbb{W}^{\left(2,4^{\star}\right)}$ and say that if $\mathcal{W}_{2 s} \in \mathbb{W}^{\left(2,4^{*}\right)}$, then this $\mathcal{W}_{2 s}$ is the tree-type $\left(2,4^{\star}\right)$-walk. We denote

$$
\dot{\mathcal{Z}}_{2 s}(n, \rho)=\sum_{\mathcal{W}_{2 s} \in \dot{W}_{2 s}} \Pi_{a, b}\left(\mathcal{W}_{2 s}\right) \cdot\left|\mathcal{C}_{\mathcal{W}_{2 s}}\right| \quad \text { and } \quad \ddot{\mathcal{Z}}_{2 s}(n, \rho)=\sum_{\mathcal{W}_{2 s} \in \ddot{\mathbb{W}}_{2 s}} \Pi_{a, b}\left(\mathcal{W}_{2 s}\right) \cdot\left|\mathcal{C}_{\mathcal{W}_{2 s}}\right| .
$$

Let us point out that two following relations are true,

$$
\left|\mathcal{C}_{\mathcal{W}_{2 s}}\right|=n^{\left|\mathbb{V}\left(\mathcal{W}_{2 s}\right)\right|}(1+o(1)), n \rightarrow \infty \quad \text { and } \quad\left|\mathcal{C}_{\mathcal{W}_{2 s}}\right| \leq n^{\left|\mathbb{V}\left(\mathcal{W}_{2 s}\right)\right|},
$$

where $\mathbb{V}\left(\mathcal{W}_{2 s}\right)$ is the ensemble of vertices of the graph $\bar{g}\left(\mathcal{W}_{2 s}\right)$.

\section{Theorem 4.1.}

Under conditions of Theorem 2.1, the following upper bounds are true

$$
\limsup _{(n, s, \rho) \rightarrow \infty} \frac{1}{n t_{s}} \dot{\mathcal{Z}}_{2 s}^{(n, \rho)} \leq 4 \exp \left\{16 V_{4} \chi\right\}
$$

and

$$
\limsup _{(n, s, \rho) \rightarrow \infty} \frac{\rho}{n t_{s}} \ddot{\mathcal{Z}}_{2 s}^{(n, \rho)} \leq C \chi \exp \left\{16 V_{4} \chi\right\}
$$


for all $0<\chi \leq \chi_{0}=\chi_{0}(U)$ and $C \geq C_{0}=C_{0}(U)$, where

$$
\chi_{0}(U)=\frac{1}{4^{11} U^{2}} \quad \text { and } \quad C_{0}(U)=3 \cdot 4{ }^{16} U^{6} .
$$

Remark. Theorem (4.1) can be proved under conditions of Theorem 1.1 with (1.5) replaced by much less restrictive condition on the probability distribution of $a_{i j}$ to be such that all its moments exist and are bounded as follows,

$$
V_{2+2 k} \leq k ! V_{2} b_{0}^{k}, \quad k=2,3, \ldots
$$

with given $b_{0}>0$ (see also [9]). In this case the constants of (4.4) should be replaced by

$$
\chi_{0}^{\prime}\left(b_{0}\right)=\frac{1}{3 \cdot 2^{19} b_{0}} \quad \text { and } \quad C_{0}^{\prime}\left(b_{0}\right)=3 \cdot 4^{16} b_{0}^{2},
$$

respectively, where we assumed that (4.5) holds with $V_{2}=1$.

To describe the general structure of the tree-type walk, let us introduce several auxiliary notions. Regarding a sub-walk of $2 a$ steps $\mathcal{W}_{2 a}$ and its graph $\bar{g}\left(\mathcal{W}_{2 a}\right)$, let us denote by $\Gamma_{\varrho}$ the ensemble of the multiple edges of $\bar{g}$ that make a connected component attached to the root $\varrho$. If the graph $\bar{g}\left(\mathcal{W}_{2 a}\right)$ has no other multiple edges than those of $\Gamma_{\varrho}$ and the first step and the last step of $\mathcal{W}_{2 a}$ are performed along the edges of $\Gamma_{\varrho}$, we say that $\mathcal{W}_{2 a}$ is the element of the block of the first level $\mathbb{B}_{a}^{(1)}(\Gamma), \Gamma=\Gamma_{\varrho}$,

$$
\mathcal{W}_{2 a}=\mathcal{B}_{a} \in \mathbb{B}_{a}^{(1)}(\Gamma) .
$$

We will say also that $\mathcal{W}_{2 s}$ by itself is a block of the first level, when no confusion can arise.

We say that a walk $\mathcal{W}_{2 b}$ is a block of the second level, $\mathcal{W}_{2 s}=\mathcal{B}_{b}^{(2)}$, if it starts and ends with the steps along the root component of multiple edges $\Gamma_{\varrho}$ and there in $\mathcal{W}_{2 b}$ there exists at least on sub-walk $\mathcal{W}_{2 a}^{\prime}$ that is the block of the first level.

By recurrence, we say that $\mathcal{W}_{2 s}$ belongs to the block of the $(k+1)$-th level, if it starts and end by the steps of $\Gamma_{\varrho}$ and contains sub-walks $\mathcal{B}^{\left(l_{1}\right)}, \ldots, \mathcal{B}^{\left(l_{p}\right)}$ such that $l_{i} \leq k$ and there exists at least one $l_{j}$ such that $l_{j}=k$.

In general, the tree-type walk $\mathcal{W}_{2 s}$ is such that its graph $\bar{g}\left(\mathcal{W}_{2 s}\right)$ represents a tree $\mathcal{T}_{h}$ of $h \geq 0$ edges and along the chronological run over $\mathcal{T}_{h}$, the sub-walks $\mathcal{B}_{a_{1}}^{\left(l_{1}\right)}, \ldots, \mathcal{B}_{a_{p}}^{\left(l_{q}\right)}$ of the levels $1 \leq l_{j} \leq s / 3$ appear at the different moments $t_{1}, \ldots t_{q}, t_{j} \in[0,2 h+1], t_{i} \neq t_{j}$.

\subsection{Proof of Theorem 4.1}

Let us introduce the weight

$$
\pi\left(\mathcal{W}_{2 s}\right)=\prod_{e \in S_{g}} \frac{V_{2 m_{e}}}{\rho^{m_{e}-1}}
$$

where $S_{\bar{g}}$ is the skeleton of the graph $\bar{g}\left(\mathcal{W}_{2 s}\right)$ and $m_{e}$ is the multiplicity of the edge $e$.

Lemma 4.1. Let us consider a family of walks $\mathbb{W}^{\diamond}(m)$ such that all edges of $\bar{g}\left(\mathcal{W}_{2 m}\right)$ are multiple and all of them are attached to the root $\varrho$. Then for any given $0<\chi \leq \chi_{0}$ and 
$C \geq C_{0}$ (4.4) the following estimate,

$$
P(m)=\sum_{\mathcal{W}_{2 m} \in \mathbb{W}_{\diamond}(m)} \pi\left(\mathcal{W}_{2 m}\right) \leq \frac{C}{4^{4 m} \rho^{1+I_{\{m \geq 3\}}}}
$$

holds for all $1 \leq m \leq s=\lfloor\chi \rho\rfloor-1$, where $\mathrm{I}_{A}=1$ if $A$ is true and $\mathrm{I}_{A}=0$ otherwise.

Proof. In the cases of $m=2,3,4$ relation (4.7) can be deduced directly from relations

$$
P(2)=\frac{V_{4}}{\rho}, \quad P(3)=\frac{V_{6}}{\rho^{2}}, \quad \text { and } \quad P(4)=\frac{3 V_{4}^{2}}{\rho^{2}} .
$$

In the general case of $m \geq 5$, we can write that

$P(m)=\sum_{l=1}^{m-5}\left(\begin{array}{c}m-1 \\ l\end{array}\right) \frac{V_{2+2 l}}{\rho^{l}} P(m-1-l)+\left(\begin{array}{c}m-1 \\ 3\end{array}\right) \frac{V_{2 m-6} V_{6}}{\rho^{m-2}}+\left(\begin{array}{c}m-1 \\ 2\end{array}\right) \frac{V_{2 m-4} V_{4}}{\rho^{m-2}}+\frac{V_{2 m}}{\rho^{m-1}}$.

Using upper bound (1.5) and assuming that (4.7) holds for $P(m-1-l)$ of the right-hand side of (4.8), we can write that

$$
P(m) \leq \frac{C}{4^{4 m} \rho^{2}} \cdot R
$$

where

$$
\begin{gathered}
R=\sum_{l=1}^{m-5} \frac{4^{4+4 l}}{l !} \cdot \frac{V_{2} U^{2 l} s^{l}}{\rho^{l}}+\frac{(m-1)(m-2)(m-3)}{3 !} \cdot \frac{4^{4 m} V_{2}^{2} U^{2 m-4}}{C \rho^{m-4}} . \\
\quad+\frac{(m-1)(m-2)}{2} \cdot \frac{4^{4 m} V_{2}^{2} U^{2 m-4}}{C \rho^{m-4}}+\frac{4^{4 m} V_{2} U^{2 m-2}}{C \rho^{m-3}} .
\end{gathered}
$$

Denoting $\phi=4^{4} U^{2} \chi$ and remembering that $V_{2}=1$, we deduce from (4.9) that

$$
R \leq 4^{4} \phi e^{\phi}+\frac{4^{16} U^{4}}{C} \phi \cdot \max _{m \geq 5} \frac{m(m-1)(m-2)}{6 m^{m-4}}+\phi^{2} \frac{4^{12} U^{4}}{C m^{m-3}} .
$$

It is easy to see that under conditions of Lemma $4.1, R \leq R_{0}<1$. Similar computations based on (4.8) show that Lemma 4.1 is true under conditions (4.5) and (4.6).

Given a Catalan tree $\mathcal{T}_{h}$, let us consider the ensemble of vertices $v_{1}, v_{2}, \ldots, v_{q}$ that have exit edges and denote by $\delta_{1}, \delta_{2} \ldots, \delta_{q}$ the number of such edges, $\delta_{i} \geq 1$. In this case we will say that the tree $\mathcal{T}_{h}$ has $q$ inner vertices with exit clusters (or bushes) $\Delta_{1}, \ldots, \Delta_{q}$ (cf. (2.5)). Given $\bar{\mu}=\left(\mu_{1}, \ldots, \mu_{q}\right)$ with $\mu_{i} \geq \delta_{i}$, let us consider the family of walks $\mathbb{W}^{\diamond}\left(\mathcal{T}_{h}, \bar{\mu}\right)$ of $2 h+2 \hat{m}$ steps, $\hat{m}=\mu_{1}+\cdots+\mu_{q}$ such that all edges of their graphs $\bar{g}\left(\mathcal{W}_{2 h+2 \hat{m}}\right)$ are multiple, $S_{\bar{g}}=\mathcal{T}_{h}$ and the vertex $v_{i}$ has $\delta_{i}+\mu_{i}$ exit edges, $1 \leq i \leq q$.

Lemma 4.2. Given $\bar{\mu}=\left(\mu_{1}, \ldots, \mu_{q}\right)$ with $\mu_{i} \geq \delta_{i}$, consider the family of walks $\mathbb{W}^{\diamond}\left(\mathcal{T}_{h}, \bar{\mu}_{q}\right)$ of $2 h+2 \hat{m}$ steps, $\hat{m}=\left|\bar{\mu}_{q}\right|=\mu_{1}+\cdots+\mu_{q}$ such that all edges of their graphs $\bar{g}\left(\mathcal{W}_{2 h+2 \hat{m}}\right)$ 
are multiple, the skeleton of $\bar{g}$ is given by $S_{\bar{g}}=\mathcal{T}_{h}$ and the vertex $v_{i}$ has $\delta_{i}+\mu_{i}$ exit edges, $1 \leq i \leq q$. Then under conditions of Lemma 4.1,

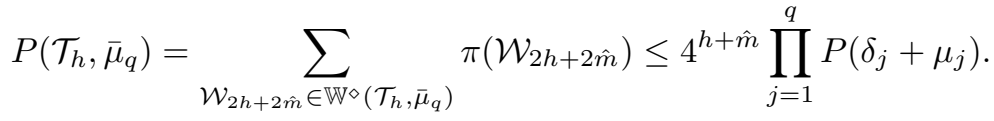

Proof. We prove Lemma 4.2 by recurrence. On the first step, let us consider the family of walks $\mathbb{W}^{\diamond}\left(p, \mu_{0}\right)$ such that each walk $\mathcal{W}_{2 p+2 \mu_{0}}$ has the graph $\bar{g}\left(\mathcal{W}_{2 p+2 \mu_{0}}\right)$ with all edges attached to the root $\rho$, the skeleton $S_{\bar{g}}$ is a bush with $p$ edges and each edge of $S_{\bar{g}}$ is multiple. Then

$$
\sum_{\mathcal{W}_{2 p+2 \mu_{0}} \in \mathbb{W}_{\diamond}\left(p, \mu_{0}\right)} \pi\left(\mathcal{W}_{2 p+2 \mu_{0}}\right) \leq P\left(p+\mu_{0}\right)
$$

and (4.10) is true. Relation (4.11) follows from the obvious observation that $\mathbb{W}^{\diamond}\left(p, \mu_{0}\right) \subset$ $\mathbb{W} \diamond\left(p+\mu_{0}\right)$.

On the next step of the recurrence, let us consider the family of walks $\mathbb{W}^{\diamond}\left(p, \mu_{0} ; \bar{\delta}_{p}, \bar{\mu}_{p}\right)$ such that their skeleton is given by a tree that has a bush with the root $\varrho$ and $p$ exit edges $\left(\varrho, v_{i}\right), 1 \leq i \leq p$ and there are $\delta_{i}>0$ main exit edges and $\mu_{i}$ additional exit edges at each $v_{i}, 1 \leq i \leq p$. Given a sub-walk $\mathcal{W}_{2 p+2 \mu_{0}} \in \mathbb{W}^{\diamond}\left(p, \mu_{0}\right)$ of $(4.11)$, we denote by $\kappa_{1}, \ldots, \kappa_{p}$ the multiplicities of the $p$ edges of its skeleton. It is not hard to see that

$$
\sum_{\mathcal{W} \in \mathbb{W} \diamond\left(p, \mu_{0} ; \bar{\delta}_{p}, \bar{\mu}_{p}\right)} \pi(\mathcal{W})=\sum_{\mathcal{W} \in \mathbb{W}^{\diamond}\left(p, \mu_{0}\right)} \pi(\mathcal{W}) \prod_{i=1}^{p}\left(\Xi^{\left(\kappa_{i}\right)}\left(\delta_{i}+\mu_{i}\right) \sum_{\mathcal{W} \in \mathbb{W}^{\diamond}\left(\delta_{i}, \mu_{i}\right)} \pi(\mathcal{W})\right)
$$

where $\Xi^{\left(\kappa_{i}\right)}\left(\delta_{i}+\mu_{i}\right)$ denotes the number of possibilities to perform $\delta_{i}+\mu_{i}$ exits from the vertex $v_{i}$ after $\kappa_{i}$ arrivals to $v_{i}$. In (4.12), we do not indicate the number of steps of corresponding walks that is obvious. Taking into account the upper estimate

$$
\Xi^{\left(\kappa_{i}\right)}\left(\delta_{i}+\mu_{i}\right)=\left(\begin{array}{c}
\delta_{i}+\mu_{i}+\kappa_{i}-1 \\
\kappa_{i}-1
\end{array}\right) \leq 2^{\delta_{i}+\mu_{i}+\kappa_{i}}
$$

and relation (4.11), we deduce from (4.12) that

$$
\begin{gathered}
\sum_{\mathcal{W} \in \mathbb{W}^{\diamond}\left(p, \mu_{0} ; \bar{\delta}_{p}, \bar{\mu}_{p}\right)} \pi(\mathcal{W}) \leq \sum_{\mathcal{W} \in \mathbb{W}^{\diamond}\left(p, \mu_{0}\right)} \pi(\mathcal{W}) \prod_{i=1}^{p}\left(2^{\delta_{i}+\mu_{i}+\kappa_{i}} \sum_{\mathcal{W} \in \mathbb{W}^{\diamond}\left(\delta_{i}, \mu_{i}\right)} \pi(\mathcal{W})\right) \\
\leq 2^{p+\mu_{0}} P\left(p+\mu_{0}\right) \prod_{i=1}^{p} 2^{\delta_{i}+\mu_{i}} P\left(\delta_{i}+\mu_{i}\right) .
\end{gathered}
$$

Remembering that $h=p+\delta_{1}+\cdot+\delta_{p}$ and $\hat{m}=\mu_{0}+\mu_{1}+\cdots+\mu_{p}$, we see that (4.10) follows from (4.14).

Now it is clear how to proceed in the general case of the walks $\mathcal{W} \in \mathbb{W}^{\diamond}(\mathcal{T} ; \bar{\mu})$ whose skeleton is given by a tree $\mathcal{T}_{h}$. It is sufficient to consider the chronological run over $\mathcal{T}_{h}$ and to find the first inner vertex $v_{0}$ such that there are no inner vertices among its children $v_{1}, \ldots, v_{p}$. 
In other words, the vertex $v_{0}$ is such that all of the exit edges $\left(v_{0}, v_{i}\right)$ are leaves and $u_{i}$ are the outer vertices.

Therefore we can apply (4.14) to the corresponding sub-walks of $\mathcal{W}^{\prime} \in \mathbb{W}^{\diamond}\left(p, \mu_{0} ; \bar{\delta}_{p}, \bar{\mu}_{p}\right)$. Then we can consider the vertex $v_{0}$ as the outer one with respect to the reduced walk $\mathcal{W} \backslash \mathcal{W}$ and repeat the reduction procedure by recurrence.

We see that in this process the vertex $v_{0}$ and the edges $\left(v^{\prime}, v_{0}\right)$ are considered twice in the estimates of the form (4.13): first in the role of $\kappa$ enters at $v_{i}$ and then in the role of $\delta+\mu$ exits from $v^{\prime}$. Therefore the base of the exponent 2 is replaced by 4 in the final estimate (4.10). We do not present the detailed computations because they repeat those of (4.12) and (4.14).

Corollary of Lemma 4.2. Using (4.7), we deduce from (4.10) that

$$
P\left(\mathcal{T}_{h}, \bar{\mu}_{q}\right) \leq \frac{C^{q}}{4^{3(h+\hat{m})} \rho^{q+\sum_{i=1}^{q} \mathrm{I}_{\left\{\delta_{i}+\mu_{i} \geq 3\right\}}}}
$$

for any $c \geq C_{0}\left(V_{2}, U\right)$.

Lemma 4.3. Let us denote by $B_{s}^{(1)}$ the sum of weights of all walks of $2 s$ steps that represent the blocks of the first level. Then

$$
B_{s}^{(1)}=\sum_{\mathcal{W}_{2 s} \in \mathbb{B}_{s}^{(1)}} \pi\left(\mathcal{W}_{2 s}\right)=\dot{B}_{s}^{(1)}+\ddot{B}_{s}^{(1)},
$$

where $\dot{B}_{s}^{(1)}$ is the sum over all walks that have only one multiple edge, this edge is the $V_{4}$-edge attached to the root,

$$
B_{s}^{(1)}=\frac{V_{4}}{\rho} T_{s-2}^{(3)},
$$

where

$$
T_{s-2}^{(3)}=\sum_{\substack{a_{1}, a_{2}, a_{3} \geq 0 \\ a_{1}+a_{2}+a_{3}=s-2}} \mathrm{t}_{a_{1}} \mathrm{t}_{a_{2}} \mathrm{t}_{a_{3}}=\mathrm{t}_{s} \frac{3 s}{2(2 s-1)} \text {. }
$$

If $\rho \geq C$, then

$$
\ddot{B}_{s}^{(1)} \leq \frac{C}{120 \rho^{2}} \mathrm{t}_{s}
$$

Proof. Taking into account the definition of the blocks of the first level and remembering that $V_{2}=1$, we can write that

$$
B_{s}^{(1)}=\sum_{h=1}^{\lfloor s / 2\rfloor} \sum_{\mathcal{T}_{h}} \sum_{\hat{m} \geq 2 h} \sum_{\begin{array}{c}
\mu_{1}, \ldots, \mu_{q} \geq 1 \\
\mu_{1}+\cdots+\mu_{q}=\hat{m}
\end{array}} P\left(\mathcal{T}_{h}, \bar{\mu}_{q}\right) T_{s-h-\hat{m}}^{(2(h+\hat{m})-1)},
$$

where the sum is taken over all possible values of $\hat{m}$ and $\mu_{i} \geq 1$ and similarly to (4.17),

$$
T_{s-h-\hat{m}}^{(2(h+\hat{m})-1)}=\sum_{\substack{a_{i} \geq 0 \\ a_{1}+\cdots+a_{2(h+\hat{m})-1}=s-h-\hat{m}}} \prod_{i=1}^{2(h+\hat{m})-1} t_{a_{i}} \leq 4^{2(h+\hat{m})} \mathrm{t}_{s} .
$$


The last inequality of (4.20) is proved in Section 5. Using (4.15), we deduce from (4.19) that

$$
\begin{gathered}
B_{s}^{(1)} \leq \mathrm{t}_{s} \sum_{h=1}^{s / 2} \sum_{\mathcal{T}_{h}} \frac{C^{q}}{4^{2 h} \rho^{q}} \prod_{i=1}^{q}\left(\sum_{\mu_{i}=1}^{\infty} \frac{1}{4^{2 \mu_{i}} \rho^{\mathrm{I}\left\{\delta_{i}+\mu_{i} \geq 3\right\}}}\right) \\
\leq \mathrm{t}_{s} \sum_{h=1}^{s / 2} \sum_{\mathcal{T}_{h}} \frac{C^{q}}{4^{2 h} \rho^{q}} \prod_{i=1}^{q}\left(\frac{1}{4^{2} \rho^{\mathrm{I}_{\left\{\delta_{i} \geq 2\right\}}}}+\sum_{\mu_{i}=2}^{\infty} \frac{1}{4^{2 \mu_{i}} \rho^{2}}\right) \leq \mathrm{t}_{s} \sum_{h=1}^{s / 2} \sum_{\mathcal{T}_{h}} \frac{C^{q}}{4^{2 h}(8 \rho)^{q}} \prod_{i=1}^{q} \frac{1}{\rho^{\mathrm{I}}\left\{\delta_{i} \geq 2\right\}} .
\end{gathered}
$$

Regarding the last expression, we observe that if $h=1$, then $q=1$ and $\delta_{1}=1$ and we get the term (4.17) in this case, and therefore $\dot{B}_{s}^{(1)} \leq 3 V_{4} \mathrm{t}_{s} /(4 \rho)$. For the remaining terms of the sum over $h \geq 2$, we observe that if $q=1$, then the tree $\mathcal{T}_{h}$ is determined uniquely with $\delta_{1} \geq 2$. Therefore we can write that

$$
\sum_{h \geq 2} \sum_{\mathcal{T}_{h}} \frac{C^{q}}{4^{2 h}(8 \rho)^{q}} \prod_{i=1}^{q} \frac{1}{\rho^{I_{\left\{\delta_{i} \geq 2\right\}}}} \leq \sum_{h \geq 2} \frac{1}{4^{2 h}}\left(\frac{C}{8 \rho^{2}}+\left(\mathrm{t}_{h}-1\right) \frac{C}{8 \rho^{2}}\right) \leq \frac{C}{120 \rho^{2}},
$$

where we have used inequality $C / \rho \leq 1$. Relation (4.18) is proved. We prove relation (4.17) in Section 5. Lemma 4.3 is proved.

Corollary of Lemma 4.3. It follows from (4.16), (4.17) and (4.18) that if $\rho \geq C$, then

$$
B_{s}^{(1)} \leq \frac{V_{4}}{\rho} \mathrm{t}_{s} .
$$

Lemma 4.4. Let us denote by $B_{s}^{(k)}$ the sum of the weights of walks that represent the blocks of the $k$-th level. If $\rho \geq C$, then the following upper bound holds,

$$
B_{s}^{(k)} \leq \frac{\alpha \mathrm{t}_{s}}{\rho} \hat{\chi}^{k-1}
$$

where $\alpha=V_{4}$ and $\hat{\chi}=128 V_{4} \chi$ with $\chi$ and $C$ determined by (4.4).

Proof. We prove Lemma 4.4 by recurrence. The case of $k=1$ is verified directly with the help of (4.22). In the general case, we can write that

$$
B_{s}^{(k+1)} \leq \sum_{p \geq 1} \sum_{a+b_{1}+\cdots+b_{p}=s} B_{a}^{(1)}\left(\begin{array}{c}
2 a-1 \\
p
\end{array}\right) p B_{b_{1}}^{(k)} \prod_{i=2}^{p}\left(B_{b_{i}}^{(1)}+B_{b_{i}}^{(2)}+\cdots+B_{b_{i}}^{(k)}\right),
$$

where $B_{a}^{(1)}$ counts the sub-walks attached to the root and $\left(\begin{array}{c}2 a-1 \\ p\end{array}\right)$ gives the upper bound of the possibilities to choose the instants to start the remaining sub-walks of $\mathcal{B}_{b_{i}}^{(l)}$; among them there is at least one block of the $k$-th level, we denote the corresponding sum by $B_{b_{1}}^{(k)}$.

Using (4.22) and assuming that (4.23) can be applied to the right-hand side of (4.24), we obtain that

$$
B_{s}^{(k+1)} \leq \sum_{p \geq 1} \sum_{a+b_{1}+\cdots+b_{p}=s} \mathrm{t}_{a} \mathrm{t}_{b_{1}} \cdots \mathrm{t}_{b_{p}} \frac{\alpha}{\rho} \cdot \frac{(2 s \alpha)^{p}}{(p-1) ! \rho^{p}} \hat{\chi}^{k-1}\left(\sum_{j=1}^{k} \hat{\chi}^{j-1}\right)^{p-1}
$$




$$
\leq \frac{\alpha}{\rho} \mathrm{t}_{s} \frac{2 \cdot 4^{2} s \alpha}{\rho} \sum_{p \geq 1} \frac{1}{p !}\left(\frac{8 \alpha \chi}{1-\hat{\chi}}\right)^{p-1} \leq \frac{\alpha \mathrm{t}_{s}}{\rho} \hat{\chi}^{k} \frac{\exp \{8 \alpha \chi /(1-\hat{\chi})\}}{4} .
$$

It is easy to deduce from (4.4) that $\hat{\chi}<1 / 2$ and $\exp \{16 \alpha \chi\}<4$. Then $B^{(k+1)} \leq \alpha \hat{\chi}^{k} \mathrm{t}_{s} / \rho$ and Lemma 4.4 is proved.

Lemma 4.5. Denote by $\ddot{B}_{s}^{(k)}$ the sum of the weights of walks that represent the blocks of $k$-th level and such that some of its sub-walks contains two or more $V_{4}$-edges that share a vertex or at least one $V_{2 l}$-edge with $l \geq 3$. Then

$$
\ddot{B}_{s}^{(k)} \leq \frac{\beta \mathrm{t}_{s}}{\rho^{2}} \hat{\chi}^{k-1}, \quad k \geq 1
$$

where $\beta=C / 120$.

Proof. Relation (4.26) with $k=1$ is verified by (4.18). In the case of $k \geq 2$, we can use the denotations of (4.24) and write that

$$
\begin{gathered}
\ddot{B}^{(k+1)} \leq \sum_{p \geq 1} \sum_{a+b_{1}+\cdots+b_{p}=s}\left(\begin{array}{c}
2 a \\
p
\end{array}\right) p\left(\ddot{B}_{a}^{(1)} B_{b_{1}}^{(k)}+B_{a}^{(1)} \ddot{B}_{b_{1}}^{(k)}\right) \prod_{i=2}^{p} \sum_{j_{i}=1}^{k} B_{b_{i}}^{\left(j_{i}\right)} \\
+\sum_{p \geq 1} \sum_{a+b_{1}+\cdots+b_{p}=s}\left(\begin{array}{c}
2 a \\
p
\end{array}\right) p(p-1) B_{a}^{(1)} B_{b_{1}}^{(k)}\left(\ddot{B}_{b_{2}}^{(1)}+\cdots+\ddot{B}_{b_{2}}^{(k)}\right) \prod_{i=3}^{p} \sum_{j_{i}=1}^{k} B_{b_{i}}^{\left(j_{i}\right)} .
\end{gathered}
$$

Using (4.18), (4.22) and (4.23) and repeating computations of (4.25), we deduce from (4.27) that

$\ddot{B}_{s}^{(k+1)} \leq \mathrm{t}_{s} \frac{\beta \hat{\chi}^{k-1}}{\rho^{2}}\left(\frac{4^{3} \alpha s}{\rho} \sum_{p \geq 1} \frac{1}{(p-1) !}\left(\frac{8 \alpha \chi}{1-\hat{\chi}}\right)^{p-1}+\left(\frac{8 \alpha s}{\rho}\right)^{2} \frac{4}{1-\hat{\chi}} \sum_{p \geq 2} \frac{1}{(p-2) !}\left(\frac{8 \alpha \chi}{1-\hat{\chi}}\right)^{p-2}\right)$.

Then we can write that

$$
\ddot{B}_{s}^{(k+1)} \leq \mathrm{t}_{s} \frac{\beta \hat{\chi}^{k}}{\rho^{2}}\left(\frac{1}{2}+\frac{2 \alpha \chi}{1-\hat{\chi}}\right) \exp \left\{\frac{8 \alpha \chi}{1-\hat{\chi}}\right\}
$$

Taking into account the conditions (4.4), it is easy to see that $\ddot{B}_{s}^{(k+1)} \leq \mathrm{t}_{s} \frac{\beta \hat{\chi}^{k}}{\rho^{2}}$.

According to the general description of the tree-type walks given in sub-section 4.1 and using the second relation of (4.1), we can write that

$$
\mathcal{Z}_{2 s} \leq n \sum_{h \geq 0} \sum_{\mathcal{T}_{h}} \sum_{p \geq 0}\left(\begin{array}{c}
2 h \\
p
\end{array}\right) \sum_{l_{1} \geq 1, \ldots, l_{p} \geq 1} \sum_{a_{1}+\cdots+a_{p}=s-h} \prod_{j=1}^{p} B_{a_{j}}^{\left(l_{j}\right)},
$$

where the sums over $l_{j}$ and $a_{i}$ are taken over all possible values such that $a_{j} \geq 2 l_{j}$. Using (4.23), we can write that for $\rho \geq C$

$$
\mathcal{Z}_{2 s} \leq n \sum_{h \geq 0} \mathrm{t}_{h} \sum_{p \geq 0} \frac{1}{p !}\left(\frac{2 s \alpha}{\rho(1-\hat{\chi})}\right)^{p} \sum_{a_{1}+\cdots+a_{p}=s-h} t_{a_{1}} \cdots t_{a_{p}} \leq 4 n \mathrm{t}_{s} \exp \left\{\frac{8 \alpha \chi}{1-\hat{\chi}}\right\} .
$$


Then the upper bound (4.2) follows.

To prove relation (4.3), we observe that if a walk $\mathcal{W}_{2 a}$ belongs to $\ddot{\mathbb{B}}_{a}^{(k)}$, then it belongs to $\mathbb{B}_{a}^{(k)}$. Therefore $\ddot{B}_{a}^{(k)} \leq B_{a}^{(k)}$ and we can write that

$$
\ddot{\mathcal{Z}}_{2 s} \leq n \sum_{h \geq 0} \sum_{p \geq 0} \sum_{\mathcal{T}_{h}} V_{2}^{h}\left(\begin{array}{c}
2 h \\
p
\end{array}\right) \sum_{l_{1} \geq 1, \ldots l_{p} \geq 1} \sum_{a_{1}+\cdots+a_{p}=s-h} p \ddot{B}_{a_{1}}^{\left(l_{1}\right)} B_{a_{2}}^{\left(l_{2}\right)} \cdots B_{a_{p}}^{\left(l_{p}\right)} .
$$

Then for sufficiently large $\rho \geq C$, we get the following upper bound,

$$
\begin{gathered}
\ddot{\mathcal{Z}}_{2 s} \leq n \sum_{h \geq 0} \frac{2 \beta s}{(1-\hat{\chi}) \rho^{2}} \mathrm{t}_{h} \sum_{p \geq 1} \frac{1}{(p-1) !}\left(\frac{2 s \alpha}{\rho(1-\hat{\chi})}\right)^{p-1} \sum_{a_{1}+\cdots+a_{p}=s-h} t_{a_{1}} \cdots t_{a_{p}} \\
\leq n \mathrm{t}_{s} \frac{8 \beta \chi}{\rho} \exp \left\{\frac{8 \alpha \chi}{1-\hat{\chi}}\right\}
\end{gathered}
$$

and relation (4.3) follows. Theorem 4.1 is proved.

\subsection{Proof of Theorem 1.1}

It follows from relations (3.2), (3.3) and Theorem 4.1 that

$$
M_{2 s_{n}}^{\left(n, \rho_{n}\right)}=\dot{\mathcal{Z}}_{2 s_{n}}^{\left(n, \rho_{n}\right)}+\ddot{\mathcal{Z}}_{2 s_{n}}^{\left(n, \rho_{n}\right)}+\tilde{\mathcal{Z}}_{2 s_{n}}^{\left(n, \rho_{n}\right)}=\dot{\mathcal{Z}}_{2 s_{n}}^{\left(n, \rho_{n}\right)}(1+o(1)) .
$$

Then the upper bound (1.6) follows from inequality (4.2). Also, it follows from Theorem 4.1 and the first relation of (4.1) that

$$
\dot{\mathcal{Z}}_{2 s_{n}}^{\left(n, \rho_{n}\right)}=n \hat{m}_{s_{n}}^{\left(\rho_{n}\right)}(1+o(1)),
$$

where

$$
\hat{m}_{s}^{(\rho)}=\sum_{\mathcal{W}_{2 s} \in \mathbb{W}_{2 s}^{\left(2,4^{*}\right)}} \pi\left(\mathcal{W}_{2 s}\right)
$$

is the total weight of $\left(2,4^{\star}\right)$-walks of $2 s$ steps.

Lemma 4.6. Denote by $\hat{F}_{\rho}(z)$ the generating function of the numbers $\hat{m}_{s}^{(\rho)}$,

$$
\hat{F}_{\rho}(z)=\sum_{s=0}^{\infty} \hat{m}_{s}^{(\rho)} z^{s}
$$

Then $\hat{F}_{\rho}(z)$ verifies the following equation,

$$
\hat{F}_{\rho}(z)=1+z\left(\hat{F}_{\rho}(z)\right)^{2}+\frac{z^{2} V_{4}}{\rho}\left(\frac{1}{1-z \hat{F}_{\rho}(z)}\right)^{4} .
$$

Proof. Given a walk $\mathcal{W}_{2 s} \in \mathbb{W}_{2 s}^{\left(2,4^{\star}\right)}$, we will say that it is of $\mathcal{M}$-type. Let us consider the first edge $e_{1}=(\varrho, \alpha)$ of the graph $\bar{g}\left(\mathcal{W}_{2 s}\right)$ of this walk. If $e_{1}$ is the $V_{2}$-edge, then $\mathcal{W}_{2 s}$ splits into three parts, the sub-walk $(\varrho, \alpha, \varrho)$ and two $\mathcal{M}$-type sub-walks $\mathcal{W}_{2 a}$ and $\mathcal{W}_{2 b}, a+b=s-1$. 
If the edge $e_{1}$ is the $V_{4}$-edge, then $\mathcal{W}_{2 s}$ splits in five parts, the sub-walk $(\varrho, \alpha, \varrho, \alpha, \varrho)$ and four sub-walks of $\mathcal{S}$-type, $\mathcal{W}_{2 a_{i}}, i=1,2,3,4$ and $a_{1}+a_{2}+a_{3}+a_{4}=s-4$. We say that the sub-walk $\mathcal{W}_{2 a}$ is of $\mathcal{S}$-type if it is an $\mathcal{M}$-type sub-walk such that its graph $\bar{g}\left(\mathcal{W}_{2 s}\right)$ has the root $\varrho^{\prime}$ attached by $V_{2}$-edges only.

Let us denote by $S_{k}$ the total weight of $\mathcal{S}$-type walks of $2 k$ steps. It is clear that

$$
S_{k}=\sum_{l=0}^{k} \sum_{a_{1}+\cdots+a_{l}=k-l} \hat{m}_{a_{1}}^{(\rho)} \cdots \hat{m}_{a_{l}}^{(\rho)} .
$$

It it easy to deduce from (4.33) that

$$
\sum_{k=0}^{\infty} S_{k} z^{k}=\frac{1}{1-z \hat{F}_{r}(z)}
$$

and then relation (4.32) follows.

Relation (4.30) combined with Lemma 4.6 proves relations (1.8) and (1.9). Theorem 1.1 is proved.

Let us consider the number $m_{s}^{(\rho)}$ and determine its part $\left[m_{s}^{(\rho)}\right]_{p}$ that contains the factor $V_{4}^{p}$. We can write that

$$
\left[m_{s}^{(\rho)}\right]_{p}=\left(\frac{V_{4}}{\rho}\right)^{p} \sum_{h \geq p}\left(\begin{array}{c}
\mathcal{T}_{h} \\
p
\end{array}\right)^{\star} T_{s-h-p}^{(2 p)}
$$

where $\left(\begin{array}{c}\mathcal{T}_{h} \\ p\end{array}\right)^{\star}$ denotes the number of possibilities to choose $p$ edges $\hat{e}_{1}, \ldots, \hat{e}_{p}$ in the tree $\mathcal{T}_{h}$ such that they do not share a common vertex. The factor $T_{s-h-p}^{(2 p)}$ counts the number of trees that can be attached to the extremities of the additional edges $\tilde{e}_{i}$ joined to $\hat{e}_{i}, i=1, \ldots, p$, respectively. Using inequality (4.20), we can deduce from (4.34) that

$$
\left[m_{s}^{(\rho)}\right]_{p} \leq \frac{1}{p !}\left(\frac{4 s V_{4}}{\rho}\right)^{p} \sum_{h \geq p} \mathrm{t}_{h} \mathrm{t}_{s-h} \leq \frac{1}{p !}\left(\frac{4 s V_{4}}{\rho}\right)^{p} \mathrm{t}_{s+1} .
$$

Then the upper bound (1.10) follows.

Regarding the right-hand sides of the upper bounds (3.30) and (3.33), it is easy to see that

$$
\frac{1}{n \mathrm{t}_{s}} \tilde{\mathcal{Z}}_{2 s}^{(n, \rho)}=O\left(\frac{s^{5}}{n}\right), \quad(n, s, r) \rightarrow \infty .
$$

This relation together with (4.29) and (4.30) implies (1.12) provided $s=o\left(n^{1 / 6}\right)$.

\section{Auxiliary statements}

In this section we collect the auxiliary statements and prove lemmas needed for the proof of Theorems 3.1 and 4.1. 


\subsection{Proof of Lemma 3.1}

Let us consider the $q$-vertex $\beta$ such that the edge of the second arrival $e_{2}=e\left(\mathfrak{a}_{2}\right)=\left(\beta, \alpha_{2}\right)$ is the minimal $q$-edge over the whole walk $\mathcal{W}_{2 s}$. We denote by $t_{2}$ the instant of time such that $e_{2}=e\left(t_{2}\right)$ and consider the sub-walk $\mathcal{W}_{\left[0, t_{2}-1\right]}$. The reasonings below concern $\mathcal{W}_{\left[0, t_{2}-1\right]}=\mathcal{W}^{*}$ only.

If the edge $\left[\beta, \alpha_{2}\right]$ represents the second distinct arrival at $\alpha_{2}$ by $\mathcal{W}^{*}$, then $\alpha_{2}$ is the blue $r$-vertex and we are done. Let us consider the case when $\left[\beta, \alpha_{2}\right]=E_{1}^{\prime}$ is the first distinct arrival at $\alpha_{2}$ by $\mathcal{W}^{*}$ and denote by $e_{\text {max }}^{\prime}=\max \left\{e, e \in E_{1}^{\prime}\left(\alpha_{2}\right)\right\}$. This edge $e_{\text {max }}^{\prime}$ is closed in $\mathcal{W}^{*}$ by a non-marked edge $f$. We consider two possible orientations of $f$ separately.

Let us consider first the cases when $f=\left(\alpha_{2}, \beta\right)$. Then $\mathcal{W}^{*}$ has to go from $\beta$ to $\alpha_{2}$ after $t(f)$ to create the $q$-edge $e\left(t_{2}\right)$. It can arrive at $\alpha_{2}$ only with by a non-marked step $h=\left(\gamma, \alpha_{2}\right)$, $\gamma \neq \beta$ that closes the marked edge $\left(\alpha_{2}, \gamma\right)=\hat{e}$. Thus, the sub-walk $\mathcal{W}^{*}$ has to go from $\beta$ to $\gamma$ to perform $h$. If $\mathcal{W}^{*}$ arrives at $\gamma$ by a marked edge $(\delta, \gamma)$, then $\gamma$ is the blue $r$-vertex because $\delta \neq \alpha_{2}$. If $\mathcal{W}^{*}$ arrives at $\gamma$ by a non-marked step $(\delta, \gamma)$, then this step closes a marked edge $\{\delta, \gamma\}$. If $\{d, \gamma\}=(\delta, \gamma)$, then $\gamma$ is the blue $r$-vertex. If $\{\delta, \gamma\}=(\gamma, \delta)$, then we get the recurrence, where the couple $a_{2}, \gamma$ is replaced by $\delta, \gamma$. Since $\varkappa_{\mathcal{W}^{*}}(\beta)=1$ by $E_{1}$, then this recurrence will be terminated before we come to $\beta$ and the $r$-vertex will be specified.

Let us consider the case when $f=\left(\beta, \alpha_{2}\right)$. To perform this step, the sub-walk $\mathcal{W}^{*}$ has to go from $\alpha_{2}$ to $\beta$ before $t(f)$. Assume that it arrives at $\beta$ by the step $h=(\gamma, \beta), \gamma \neq \alpha_{2}$ that has to be the non-marked one.

Let us consider first the case when $\gamma \neq \alpha_{1}$. The sub-walk has to go from $\alpha_{2}$ to $\gamma$ and arrive at $\gamma$ by the step $g=(\delta, \gamma)$. If this step is marked, then $\gamma$ is the blue $r$-vertex and we are done. If $g$ is non-marked, then it closes the marked edge $\{\gamma, \delta\}$. If $\{\gamma, \delta\}=(\delta, \gamma)$, then $\gamma$ is the blue $r$-vertex. If $\{\gamma, \delta\}=(\gamma, \delta)$, then we get a recurrence. Since $\varkappa_{\mathcal{W}^{*}}\left(\alpha_{2}\right)=1$ by $E_{1}$, then this recurrence will be terminated by a blue $r$-vertex.

Finally, let us consider the case when $\gamma=\alpha_{1}$ and $h=\left(\alpha_{1}, \beta\right)$. Then the sub-walk has to go from $\alpha_{2}$ to $\alpha_{1}$ and arrive it by the step $g=\left(\gamma, \alpha_{1}\right)$. If this step is marked, then $\alpha_{1}$ is the blue $r$-vertex. If $g$ is non-marked, then either $\gamma=\epsilon$ or $\gamma \neq \epsilon$, where the edge $\left(\epsilon, \alpha_{1}\right) \in E_{1}\left(\alpha_{1}\right)$.

If $g=\epsilon$, then we get a recurrence with the couple $\alpha_{1}, \beta$ replaced by $\epsilon, \alpha_{1}$. Please note that the fact that $\left(\epsilon, \alpha_{1}\right)$ generally is not the first arrival at $\alpha_{1}$ does not alter this recurrence. Then we terminate with the blue $r$-edge.

If $\gamma \neq \epsilon$, then $g$ closes a marked edge $\left\{\gamma, \alpha_{1}\right\}$. If $\left\{\gamma, \alpha_{1}\right\}=\left(\gamma, \alpha_{1}\right)$, then $\alpha_{1}$ is the blue $r$-vertex. If $\left\{\gamma, \alpha_{1}\right\}=\left(\alpha_{1}, \gamma\right)$, then we get a recurrence that will terminate before $\alpha_{2}$ and the blue $r$-vertex will be specified. Lemma 3.1 is proved.

\subsection{Catalan trees and exponential estimates}

In papers $[9,12]$, the following statement is proved with the help of recurrent relation (2.6).

Lemma 5.1. Consider the family of Catalan trees constructed with the help of $s$ edges and such that the root vertex $\varrho$ has $d$ edges attached to it and denote by $\mathrm{t}_{s}^{(d)}$ its cardinality,

$$
\mathrm{t}_{s}^{(d)}=\sum_{u_{1}+\cdots+u_{d-1}+u_{d}=s-d} \mathrm{t}_{u_{1}} \mathrm{t}_{u_{2}} \cdots \mathrm{t}_{u_{d-1}} \mathrm{t}_{u_{d}}
$$


where the sum runs over all possible $u_{i} \geq 0$. Then the upper bound

$$
\mathrm{t}_{s}^{(d)} \leq e^{-\eta d} \mathrm{t}_{s}, \quad \eta=\ln (4 / 3)
$$

is true for any given integers $d$ and $s$ such that $1 \leq d \leq s$.

Remark. We can say that $\mathrm{t}_{s}^{(d)}$ represents the number of Catalan trees such that their root vertex $\varrho$ has the exit sub-cluster of cardinality $d$. It is not hard to deduce from (2.6) that the numbers $\left\{\mathrm{t}_{s}^{(d)}, 1 \leq d \leq s\right\}$ verify the following recurrent relation [9],

$$
\mathrm{t}_{s}^{(d)}=\mathrm{t}_{s}^{(d-1)}-\mathrm{t}_{s-1}^{(d-2)}, \quad 3 \leq d \leq s
$$

with the initial values $\mathrm{t}_{s}^{(1)}=\mathrm{t}_{s-1}, s \geq 1$ and $\mathrm{t}_{s}^{(2)}=\mathrm{t}_{s-1}, s \geq 2$. Denoting $\mathrm{t}_{s}^{(d)}=T_{s-d}^{(d)}$ (4.17) and changing variables by $k=s-d, p=d$, we deduce from (5.1) and an elementary consequence of (1.7) $\mathrm{t}_{s+1} \leq 4 \mathrm{t}_{s}, s \geq 0$ the following inequalities,

$$
T_{k}^{(p)} \leq e^{-\eta p} \mathrm{t}_{k+p} \leq 4^{p} \mathrm{t}_{k} .
$$

This proves the upper bound (4.20).

Regarding the numbers $R_{s}=T_{s-2}^{(3)}(4.17)$, it is easy to see that

$$
\sum_{s=2}^{\infty} R_{s} z^{s}=z^{2} f^{3}(z)
$$

It follows from (1.11) that $z f^{2}(z)=f(z)-1$. Using this equality several times, we obtain that

$$
R_{s}=3 \frac{(2 s-2) !}{(s-2) !(s+1) !}, \quad s \geq 2 .
$$

This proves the last relation of (4.17).

Let us denote by $\mathcal{N}_{s}^{(1,2)}$ the number of tree-type walks of $2 s$ steps such that their graph contains one $V_{4}$-edge $(\alpha, \beta)$ and remaining $s-2$ edges are $V_{2}$-edges. Then it is not hard to see that (cf. (4.17))

$$
\mathcal{N}_{s}^{(1,2)}=\sum_{a+b_{1}+2+b_{3}=s-2}(2 a+1) \mathrm{t}_{a} \mathrm{t}_{b_{1}} \mathrm{t}_{b_{2}} \mathrm{t}_{b_{3}}, \quad s \geq 2,
$$

where the factor $(2 a+1)$ gives the number of choice of the root $\rho$ in the sub-tree $\mathcal{T}_{a}$ attached to the vertex $\alpha$ while the remaining three sub-trees $\mathcal{T}_{b_{i}}$ are attached to the vertex $\beta$. Then the generating function

$$
\Phi^{(1,2)}(z)=\sum_{s=2}^{\infty} \mathcal{N}_{s}^{(1,2)} z^{s}, \quad \mathcal{N}_{2}^{(1,2)}=1
$$

is given by expression

$$
\Phi^{(1,2)}(z)=2 z^{3} f^{\prime}(z) f^{3}(z)+z^{2} f^{4}(z) .
$$

Using (1.11), one can show that (see [12] for details)

$$
\mathcal{N}_{s}^{(1,2)}=\frac{(2 s) !}{(s-2) !(s+2) !}=s \mathrm{t}_{s}\left(1-\frac{3}{s+2}\right), \quad s \geq 2 .
$$


More generally, denoting by $\mathcal{N}_{s}^{(1, m)}$ the number of even closed walks $\mathcal{W}_{2 s}$ such that their graphs contain one edge of total multiplicity $2 m$ and all other edges of multiplicity 2 , one can prove that relation

$$
\mathcal{N}_{s}^{(1, m)}=\frac{(2 s) !}{(s-m) !(s+m) !}, \quad s \geq m \geq 1
$$

is true. The proof of (5.3) can be obtained with the help of the following reasoning similar to that used in [9] to deduce relation (5.2).

Let us introduce variables

$$
D_{k}^{(m)}=\sum_{a+b_{1}+\cdots+b_{2 m-1}=k-m}(2 a+1) \mathrm{t}_{a} \mathrm{t}_{b_{1}} \cdots \mathrm{t}_{2 m-1}, \quad k \geq m \geq 1,
$$

such that $D_{k}^{(l)}=0$ for all $0 \leq l<k$ and

$$
E_{k}^{(m)}=\sum_{a+b_{1}+\cdots+b_{2 m-1}=k-m}(2 a+1) \mathrm{t}_{a} \mathrm{t}_{b_{1}} \cdots \mathrm{t}_{2 m}, \quad k \geq m \geq 1,
$$

such that $E_{k}^{(l)}=0$ for all $0 \leq l<k$. It is not hard to see that $D_{s}^{(m)}=\mathcal{N}_{s}^{(1, m)}$. Indeed, regarding a vertex $\alpha$, we attach to it $m$ blue edges and get the sub-cluster $\Delta_{m}$. Then we attach to $\alpha$ a green Catalan tree $\mathcal{T}_{a}$ and determine the root $\varrho$ by choosing one of $2 a+1$ instants of time of the chronological run over $\mathcal{T}_{a}$. To we attach $2 m-1$ red Catalan trees $\mathcal{T}_{b_{i}}$ at the remaining $2 m-1$ instants of time of the chronological run over the sub-cluster $\Delta_{m}$ of blue edges.

According to these definitions, we get that

$$
D_{k}^{(1)}=\sum_{a+b=k-1}(2 a+1) \mathrm{t}_{a} \mathrm{t}_{b}, \quad k \geq 1
$$

and $D_{0}^{(1)}=0$. Then the generating function $\mathcal{D}^{(1)}(z)=\sum_{k \geq 0} D_{k}^{(1)} z^{k}$ is such that

$$
\mathcal{D}^{(1)}(z)=2 z^{2} f^{\prime}(z) f(z)+z(f(z))^{2}=z f^{\prime}(z)
$$

and therefore

$$
D_{k}^{(1)}=\frac{(2 k) !}{(k-1) !(k+1) !}, \quad k \geq 1 .
$$

In this computation, we have used the identity $2 z f^{\prime}(z) f(z)=f^{\prime}(z)-(f(z))^{2}$ that follows from (1.11). We can also wright that $D_{k}^{(1)}=k \mathrm{t}_{k}$. This relation is obvious because the number $D_{k}^{(1)}$ by its definition enumerates the ensemble of Catalan trees with one marked edge colored in blue.

It follows from the definition of $E_{k}^{(m)}$ that the generating function $\mathcal{E}^{(1)}(z)=\sum_{k \geq 0} E_{k}^{(1)} z^{k}$ is given by the formulas

$$
\mathcal{E}^{(1)}(z)=2 z^{2} f^{\prime}(z)(f(z))^{2}+z(f(z))^{3}=\frac{f^{\prime}(z)}{2}-\frac{f(z)-1}{2 z}
$$

and therefore

$$
E_{k}^{(1)}=\frac{k}{2} \mathrm{t}_{k+1}, \quad k \geq 0
$$


Using the fundamental recurrence (2.6), we can write that

$$
\begin{gathered}
D_{k}^{(m)}=\sum_{a+b_{1}+\cdots+b_{2 m-3}+c=k-m}(2 a+1) \mathrm{t}_{a} \mathrm{t}_{b_{1}} \cdots \mathrm{t}_{2 m-3} \sum_{b_{2 m-2}+b_{2 m-1}=c} \mathrm{t}_{2 m-2} \mathrm{t}_{2 m-1} \\
=\sum_{a+b_{1}+\cdots+b_{2 m-3}+c=k-m}(2 a+1) \mathrm{t}_{a} \mathrm{t}_{b_{1}} \cdots \mathrm{t}_{2 m-3} \mathrm{t}_{c+1} .
\end{gathered}
$$

Then we get the following equality,

$$
D_{k}^{(m)}=E_{k}^{(m-1)}-D_{k}^{(m-1)}, \quad k \geq m \geq 2 .
$$

Similar computation shows that

$$
E_{k}^{(m)}=D_{k+1}^{(m)}-E_{k}^{(m-1)}, \quad k \geq m \geq 2 .
$$

Using these recurrent relations together with the initial expressions given by $D_{k}^{(1)}$ and $E_{k}^{(1)}$, one can easily check that

$$
D_{k}^{(m)}=\frac{(2 k) !}{(k-m) !(k+m) !} \quad \text { and } \quad E_{k}^{(m)}=\frac{(2 k+1) !}{(k+1-m) !(k+m) !}
$$

for all $k \geq m \geq 1$. This proves relation (5.3).

Using the same reasoning as above, it is not hard to see that relation (5.2) implies that

$$
\mathrm{t}_{s}^{(d)}= \begin{cases}(2 m-1) \mathrm{t}_{s-m} \prod_{i=1}^{m-1} \frac{s+1-m-i}{s+1-i}, & \text { if } d=2 m-1, m \geq 1, \\ m \mathrm{t}_{s-m} \prod_{i=1}^{m-1} \frac{s-m-i}{s+1-i}, & \text { if } d=2 m, m \geq 0 .\end{cases}
$$

Let us denote by $\mathcal{N}_{s}^{(2,2)}$ the number of tree-type walks that contain two $V_{4}$-edges while the remaining ones are the $V_{2}$-edges. One can show that the generating function $\Phi_{s}^{(2,2)}$ is given by the following expression,

$$
\Phi^{(2,2)}(z)=\frac{z^{4}}{2} f^{\prime \prime}(z) f^{4}(z)+3 z^{4} f^{\prime}(z) f^{6}(z)
$$

Then

$$
\mathcal{N}_{s}^{(2,2)}=\mathrm{t}_{s} \frac{k^{4}+8 k^{3}+39 k^{2}+12}{2(k+2)(k+3)}-4^{k}+3 \frac{(2 k) !}{(k-4) !(k+4) !} .
$$

Although the last expression is not so compact as those of (5.3), we can easily deduce from them that

$$
\lim _{s \rightarrow \infty} \frac{1}{s \mathrm{t}_{s}} \mathcal{N}_{s}^{(1,2)}=1 \quad \text { and } \quad \lim _{s \rightarrow \infty} \frac{1}{s^{2} \mathrm{t}_{s}} \mathcal{N}_{s}^{(2,2)}=\frac{1}{2} .
$$

These relations agree with the upper bounds (4.35) in the cases of $p=1$ and $p=2$. Moreover, one can put forward a conjecture that

$$
\mathcal{N}_{s}^{(p, 2)}=\frac{s^{p}}{p !} \mathrm{t}_{s}(1+o(1)), \quad s \rightarrow \infty .
$$


This allows one to expect that the following lower bound holds (cf. (1.10)),

$$
\liminf _{(n, s, \rho) \rightarrow \infty} \frac{1}{\mathrm{t}_{s}} \hat{m}_{s}^{(\rho)} \geq e^{\chi V_{4}} .
$$

Finally, let us note that a part of $\mathcal{N}_{s}^{(2,2)}$ given by

$$
\ddot{\mathcal{N}}_{s}^{(2,2)}=4 \frac{(2 s) !}{(s-4) !(s+4) !}
$$

represents the number of $(2,4)$ walks of $2 s$ steps such that have two $V_{4}$-edge with common vertex. It is easy to see that $\ddot{\mathcal{N}}_{s}^{(2,2)}=s \mathrm{t}_{s}(1+o(1))$ and therefore these walks do not contribute to the asymptotic expression for $M_{2 s}^{(\rho)}$. This is in complete accordance with the definition of the numbers $\hat{m}_{s}^{(\rho)}$ as the total weight of the tree-type $\left(2,4^{\star}\right)$-walks. The terms $\ddot{\mathcal{N}}_{s}^{(2,2)}$ and $\mathcal{N}_{s}^{(1,3)}$ provide the leading contribution to the asymptotic expansion (1.13).

\subsection{D-lemma}

In the present subsection we prove Lemma 3.4. Let us introduce an auxiliary collection of variables

$$
\mathcal{H}=\left(\bar{m}_{I},\left(\bar{\Lambda}, \Psi, \bar{f}^{\prime \prime}\right)_{J},\left(\Phi, \bar{f}^{\prime}\right)_{K}\right)
$$

that represents a part of parameters $\mathcal{P}_{R}(\bar{x}, \bar{y}, \bar{z}, \mathcal{H})(3.17)$ and consider its numerical realization $\langle\mathcal{H}\rangle$. Then relation $(3.24)$ can be rewritten in the following form,

$$
\left|\sqcup_{\langle\mathcal{H}\rangle} \mathbb{W}_{2 s}\left(D,\left\langle\breve{v} \uplus \mathcal{G}^{(c)}\right\rangle_{s},\langle\mathcal{H}\rangle, \Upsilon\right)\right| \leq 2^{|\bar{q}|} D^{|\bar{p}|}\left(e^{\eta} h_{0}^{2}\right)^{I+J+K} e^{-\eta D+e D / h_{0}} \mathrm{t}_{s},
$$

where the disjoint union is taken over the set of all possible realizations $\mathbb{H}=\{\langle\mathcal{H}\rangle\}$. By the construction, the values of $\langle(\bar{x}, \bar{y}, \bar{z})\rangle_{s}$ are determined by the realization of the color dia$\operatorname{gram}\left\langle\mathcal{G}^{(c)}\right\rangle_{s}$. As we will see below, the set $\left(\langle\bar{x}, \bar{y}, \bar{z}\rangle_{s},\langle\mathcal{H}\rangle\right)$ uniquely determine the nest cells $\check{v}_{1}, \ldots, \check{v}_{R}$ in the underlying trees $\mathcal{T}\left(\mathcal{W}_{2 s}\right)$ where the clusters $\check{\Delta}_{1}, \ldots, \check{\Delta}_{R}$ are attached. Then we can apply inequalities of the form (5.3) to get the upper bound of the set of underlying trees that is is exponential with respect to the sum $\sum_{i=1}^{R} d_{i}, d_{i}=\left|\check{\Delta}_{i}\right|$.

We prove (5.5) by recurrence with respect to $N=I+J+K$.

\subsubsection{The case of $R=1$}

If the total number of cells at $\breve{\beta}$ is equal to one, $R=1$, then either $\mathcal{P}_{1}=x_{1}$ or $\mathcal{P}_{1}=z_{1}$ and the set of variables $\mathcal{H}$ is empty. For simplicity, we consider the former case such that $\left\langle x_{1}\right\rangle_{s}=\tau_{1} \geq 1$. Regarding a walk $\mathcal{W}_{2 s}$ from the left-hand side of (5.5) and the corresponding tree $\mathcal{T}_{s}=\mathcal{T}\left(\mathcal{W}_{2 s}\right)$, we observe that its vertex $\check{v}$ such that $\check{v}=\mathfrak{R}\left(\xi_{\tau_{1}}\right)$ is attached by a subcluster $\check{\Delta}_{1}$ of $d_{1}$ edges. It is easy to construct the corresponding family of trees $\mathbb{T}_{s}\left(\tau_{1}, d_{1}\right)$ with the help of the following procedure.

Let us take a root vertex $\mathfrak{b}_{0}$ and attach to it a linear branch $\mathcal{B}_{l}$ that consists of $l$ edges and $l+1$ vertices $\mathfrak{b}_{0}, \mathfrak{b}_{1}, \ldots, \mathfrak{b}_{l}$. Regarding the set of vertices $\left\{\mathfrak{b}_{0}, \ldots, \mathfrak{b}_{l-1}\right\}$, we attach to them the sub-trees $\mathcal{T}_{a_{1}}, \ldots, \mathcal{T}_{a_{l}}$ with given $\bar{a}=\left(a_{1}, \ldots, a_{l}\right)$ such that $|\bar{a}|=a_{1}+\cdots+a_{l}=\tau_{1}-l$. We do this in the way that the sub-trees grow to the left of the branch $\mathfrak{B}_{l}$ with respect to the ascending chronological run over $\mathfrak{B}_{l-1}$ from $\mathfrak{b}_{0}$ to $\mathfrak{b}_{l-1}$. 
We attach to the vertex $\check{v}=\mathfrak{b}_{l}$ the sub-cluster $\check{\Delta}_{1}$ of $D=d$ edges. Using $d$ vertices of $\check{\Delta}_{1}$, we attach to them $d$ sub-trees $\mathcal{T}_{b_{1}}, \ldots, \mathcal{T}_{b_{d}}$. Regarding the vertices $\mathfrak{b}_{l_{1}}, \ldots, \mathfrak{b}_{0}$ as the descending part of the chronological run over $\mathcal{B}_{l-1}$, we construct on these vertices the sub-trees $\mathcal{T}_{c_{1}}, \ldots, \mathcal{T}_{c_{l}}$, where $b_{i}$ and $c_{j}$ are such that $\sum_{i=1}^{d} b_{i}+\sum_{j=1}^{l} c_{j}=s-\tau_{1}-d$.

Then we can write that

$$
\mathrm{T}_{s}\left(\tau_{1}, d\right)=\left|\mathbb{T}_{s}\left(\tau_{1}, d\right)\right|=\sum_{l=1}^{\tau_{1}} \sum_{\substack{\bar{a}: \\|\bar{a}|=\tau_{1}-l}} \mathrm{t}_{a_{1}} \cdots \mathrm{t}_{a_{l}} \sum_{\substack{\bar{b}, \bar{c}: \\|\bar{b}|+|\bar{c}|=s-\tau_{1}-d}} \mathrm{t}_{b_{1}} \cdots \mathrm{t}_{b_{d}} \cdot \mathrm{t}_{c_{1}} \cdots \mathrm{t}_{c_{l}} .
$$

It follows from Lemma 5.1 that

$$
\sum_{\bar{b}:|\bar{b}|=m} \mathrm{t}_{b_{1}} \cdots \mathrm{t}_{b_{d}} \leq e^{-\eta d} \mathrm{t}_{m+d}
$$

Then we can deduce from (5.6) the following inequality,

$$
e^{\eta d} \mathrm{~T}_{s}\left(\tau_{1}, d\right) \leq \sum_{l=1}^{\tau_{1}} \sum_{\substack{\bar{a}, b, \bar{c}:|\bar{a}|, b|| \bar{c}|\geq 0\\| \bar{a}|+b+| \bar{c} \mid=s-l}} \mathrm{t}(\bar{a}) \cdot \mathrm{t}_{b} \cdot \mathrm{t}(\bar{c})=\mathrm{t}_{s},
$$

where we denoted $\mathrm{t}(\bar{a})=\mathrm{t}_{a_{1}} \cdots \mathrm{t}_{a_{l}}$. The last equality follows from the observation that the sum in the central part of (5.8) represents the cardinality of the set of Catalan trees $\mathcal{T}_{s}$ that have the vertex $\check{v}$ seen at the instant $\xi_{\tau_{1}}$ of the chronological run $\mathcal{R}\{\mathcal{T}\}$ colored in white, the others being the black ones. Clearly, given $\mathcal{T}_{s}$, there exists only one such vertex and therefore the cardinality of this family is equal to $\mathrm{t}_{s}$.

Combining inequality (5.8) with the filtration estimate (3.9a), we get the needed upper bound (3.9b),

$$
\left|\mathbb{W}_{2 s}\left(D, \breve{v}\left(x_{1}\right) \uplus\left\langle\mathcal{G}^{(c)}(\bar{\nu}, \bar{p}, \bar{q})\right\rangle_{s}^{(b)}, \Upsilon\right)\right| \leq 2^{|\bar{q}|} D^{|\bar{p}|} e^{-\eta D} \mathrm{t}_{s} .
$$

\subsubsection{The cases of $R=2, N=2$}

a) Let $\mathcal{P}_{2}$ be such that $\bar{x}=\left(x_{1}, x_{2}\right)$ and $\langle\bar{x}\rangle_{s}=\left(\tau_{1}, \tau_{2}\right), 1 \leq \tau_{1}<\tau_{2}$. Then $\mathcal{H}$ is empty. The mirror cells are not present at $\breve{\beta}$ and therefore the tree $\mathcal{T}_{s}=\mathcal{T}\left(\mathcal{W}_{2 s}\right)$ is such that the vertices $\check{v}_{1}$ and $\check{v}_{2}$ lie on two different branches $\mathcal{B}_{1}$ and $\mathcal{B}_{2}$, respectively. Let us describe the construction of the corresponding subset of trees and estimate its cardinality.

We start with the first branch $\mathcal{B}_{1}=\mathcal{B}_{l_{1}}$ that contains $l_{1}$ edges and $l_{+} 1$ vertices $\mathfrak{b}_{0}, \mathfrak{b}_{1}, \ldots, \mathfrak{b}_{l_{1}}$ and construct on its first $l_{1}$ vertices the sub-trees $\mathcal{T}_{1}, \ldots, \mathcal{T}_{a_{l_{1}}}$ such that $|\bar{a}|=\tau_{1}-l_{1}$. Then we attach to $\mathfrak{b}_{l_{1}}=\check{v}_{1}$ the sub-cluster $\check{\Delta}_{1}$ of $d_{1}$ edges and join to each of $d_{1}$ vertices the sub-trees $\mathcal{T}_{b_{1}^{(1)}}, \ldots, \mathcal{T}_{b_{d_{1}}^{(1)}}$ such that $\left|\bar{b}^{(1)}\right|=m_{1} \geq 0$.

Performing $\lambda$ steps down along the descending part of $\mathcal{B}_{1}$, we stop at the vertex $\mathfrak{b}_{l_{1}-\lambda}$, $1 \leq \lambda \leq l_{1}$ and attach to it the second branch $\mathcal{B}_{2}$ with vertices $\mathfrak{c}_{0}=\mathfrak{b}_{l_{1}-\lambda}, \mathfrak{c}_{1}, \ldots, \mathfrak{c}_{l_{2}}$.

Regarding vertices $\mathfrak{b}_{l_{1}-1}, \ldots, \mathfrak{b}_{l_{1}-\lambda}, \mathfrak{c}_{1}, \ldots \mathfrak{c}_{l_{2}-1}$, we construct attach to them the sub-trees $\mathcal{T}_{c_{j}^{(1)}}$ such that $\left|\bar{c}^{(1)}\right|=c_{1}^{(1)}+\ldots c_{\lambda}^{(1)}+c_{\lambda+1}^{(1)}+\cdots+c_{\lambda+l_{2}-1}^{(1)}=\tau_{2}-\tau_{1}-l_{1}-l_{2}-m_{1}$.

We construct the second sub-cluster $\check{\Delta}_{d_{2}}$ with $\left|\check{\Delta}_{d_{2}}\right|=d_{2}$ on the vertex $\mathfrak{c}_{l_{2}}=\check{v}_{2}$ and construct on the $d_{2}$ vertices obtained the sub-trees $\mathcal{T}_{b_{1}^{(2)}}, \ldots, \mathcal{T}_{b_{d_{2}}^{(2)}}$. Finally, we join the sub- 
trees $\mathcal{T}_{c_{1}^{(2)}}, \ldots, \mathcal{T}_{c_{l_{1}+l_{2}-\lambda}^{(2)}}$ to the vertices $\left\{\mathfrak{c}_{l_{2}-1}, \ldots, \mathfrak{c}_{1}, \mathfrak{b}_{l_{1}-\lambda}, \ldots, \mathfrak{b}_{0}\right\}$. Then we can write that

$$
\begin{array}{r}
\left|\mathbb{T}_{s}\left(\tau_{1}, d_{1} ; \tau_{2}, d_{2}\right)\right|=\sum_{l_{1}=1}^{\tau_{1}} \sum_{\lambda=1}^{l_{1}} \sum_{l_{2}=1}^{\tau_{2}-l_{1}-d_{1}} \sum_{\bar{a}:|\bar{a}|=\tau_{1}-l_{1}} \mathrm{t}(\bar{a}) \sum_{m_{1}=0}^{\tau_{2}-\tau_{1}-l_{1}} \sum_{\bar{b}^{(1)}:\left|\bar{b}^{(1)}\right|=m_{1}} \mathrm{t}\left(\bar{b}^{(1)}\right) \\
\times \sum_{\bar{c}^{(1)}:\left|\bar{c}^{(1)}\right|=0}^{\tau_{2}-\tau_{1}-d_{1}-m_{1}-l_{2}} \mathrm{t}\left(\bar{c}^{(1)}\right) \sum_{m_{2}=0}^{s-\tau_{2}-d_{2}} \sum_{\bar{b}^{(2)}:\left|\bar{b}^{(2)}\right|=m_{2}} \mathrm{t}\left(\bar{b}^{(2)}\right) \sum_{\bar{c}^{(2)}:\left|\bar{c}^{(2)}\right|=s-\tau_{2}-d_{2}-m_{2}} \mathrm{t}\left(\bar{c}^{(2)}\right) .
\end{array}
$$

We apply (5.7) two times with respect to the sub-trees with the exit sub-clusters $\check{\Delta}_{1}$ and $\check{\Delta}_{2}$ and get the estimate

$$
\sum_{\bar{b}^{(1)}:\left|\bar{b}^{(1)}\right|=m_{1}} \mathrm{t}\left(\bar{b}^{(1)}\right) \sum_{\bar{b}^{(2)}:\left|\bar{b}^{(2)}\right|=m_{2}} \mathrm{t}\left(\bar{b}^{(2)}\right) \leq e^{-\eta\left(d_{1}+d_{2}\right)} \mathrm{t}_{m_{1}+d_{1}} \mathrm{t}_{m_{2}+d_{2}}
$$

The same reasoning as before show that inequality of the form (5.8) with $d=d_{1}+d_{2}$, where $\mathrm{t}_{s}$ represents the number of Catalan trees such that the vertices seen at the instants $\xi_{\tau_{1}}$ and $\xi_{\tau_{2}}$ are colored in white. Using (3.23), it is easy to complete the proof of (5.5).

b) Let us consider the case of two cells $\mathcal{P}_{2}=\left(x_{1},\left(y_{1}, \Lambda\right)\right)$ such that $\left\langle\left(x_{1}, x_{2}\right)\right\rangle_{s}=\left(\tau_{1}, \tau_{2}\right)$ are given as well as a particular value $\langle\Lambda\rangle=\lambda^{\prime}$. Then $\breve{\beta}$ is attributed by one proper cell and one imported cell. We first study the case when $y_{1}$ does not fill the edge-box attached to a red or to a green vertex of $\mathcal{G}^{(c)}$. There is no mirror cells at $\breve{\beta}$ and therefore the vertices $\check{v}_{1}=\mathfrak{R}\left(\xi_{\tau_{1}}\right)$ and $\check{v}_{2}=\mathfrak{R}\left(\xi_{\tau_{2}}\right)$ are situated on different branches of $\mathcal{T}_{s}$. Let us briefly describe the construction of the corresponding tree $\mathcal{T}_{s}$ that is very similar to that we performed above.

Taking the root vertex $\mathfrak{b}_{0}$, we draw a branch $\mathcal{B}_{1}$ with the help of $l_{1}$ edges. Starting from the extreme vertex $\mathfrak{b}_{l_{1}}$, we descend by $\lambda_{1}$ steps till the vertex $\mathfrak{b}_{l_{1}-\lambda_{1}}$ and attach to it the second branch $\mathfrak{B}_{2}$ of $l_{2}$ edges. We attach to the vertex $\check{v}_{1}$ the sub-cluster $\Delta_{1}$ of $d_{1}$ edges. We denote the skeleton obtained by $K\left(l_{1}, d_{1}, \lambda_{1} ; l_{2}\right)$. Regarding the vertices of $K$, we construct on them the subtrees $\mathcal{T}(\bar{a}), \mathcal{T}\left(\bar{m}_{1}\right), \mathcal{T}(\bar{b})$ and $\mathcal{T}\left(\bar{c}^{(1)}\right)$ with properly chosen values. In this construction, we do not use the vertices of the descending part of $K$ from the vertex $\check{v}_{2}$ to $\mathfrak{b}_{0}$. We denote the sub-tree obtained by $\grave{\mathcal{T}}=\mathcal{T}\left(\bar{a}, \bar{\beta}, \bar{c}^{(1)}, \bar{m}_{1} ; K\right)$.

Now let us consider a sub-walk $\mathcal{W}_{\left[0, \xi_{\tau_{2}-1}\right]}(\grave{\mathcal{T}})=\grave{\mathcal{W}}$ performed according to the rules of $\left\langle\mathcal{G}^{(c)}\right\rangle_{s}$ and $\Upsilon$. The vertex $\breve{\beta}$ is completely determined by the run of $\mathcal{W}$ as well as the vertex $\mathcal{W}\left(\xi_{\tau_{2}}\right)=\gamma$. Therefore the path $\mathcal{L}$ from $\gamma$ to $\breve{\beta}$ by non-marked steps according to $\Upsilon$, if it exists, is completely determined as well as its length $|\mathcal{L}|=\lambda_{2}$. This produces the indicator function $I_{\mathcal{W}}\left(\lambda^{\prime}\right)$ that is equal to 1 if $\lambda^{\prime}=\lambda_{2}$ and zero otherwise.

With $\lambda_{2}$ determined, we descend from $\check{v}_{2}$ to $\mathfrak{b}_{0}$ of $K$ by $\lambda_{2}$ steps and attach to the vertex obtained the sub-cluster $\Delta_{2}$ of $d_{2}$ edges. Then the family of sub-trees $\mathcal{T}\left(d_{2}\right)=\mathcal{T}_{s-\tau_{2}}\left(\bar{c}^{(2)}, \bar{m}_{2}\right)\left(d_{2}\right)$ with the help of the remaining edges is constructed.

Regrading the sum over all values of $\langle\mathcal{H}\rangle=\lambda^{\prime}$, we see that the cardinality of the set of trees obtained is given by the following expression (cf. (5.10)),

$$
\sum_{\lambda^{\prime}} \sum_{l_{1}, \lambda_{1}, l_{2}} \sum_{K\left(l_{1}, d_{1}, \lambda_{1} ; l_{2}\right)} \sum_{\grave{\mathcal{T}}} I_{\grave{\mathcal{W}}}\left(\lambda^{\prime}\right) \cdot \sum_{\dot{\mathcal{T}}} 1 .
$$


Taking into account that

$$
\sum_{\hat{\mathcal{T}}_{s-\tau_{2}}\left(\bar{c}^{(2)}, \bar{m}_{2}\right)\left(d_{2}\right)} 1 \leq e^{-\eta d_{2}} \sum_{\mathcal{T}_{s-\tau_{2}}\left(\bar{c}^{(2)}, \bar{m}_{2}\right)} 1
$$

and that $\sum_{\lambda^{\prime}} I_{\grave{\mathcal{W}}}\left(\lambda^{\prime}\right)=1$, we conclude that the right-hand side of $(5.12)$ is bounded by the sum

$$
e^{-\eta d_{2}} \sum_{l_{1}, \lambda_{1}, l_{2}} \sum_{K\left(l_{1}, d_{1}, \lambda_{1} ; l_{2}\right)} \sum_{\dot{\mathcal{T}}} \sum_{\dot{\mathcal{T}}} 1 \leq e^{-\eta\left(d_{1}+d_{2}\right)} \mathrm{t}_{s} .
$$

To get the last inequality, we have used the same reasoning as that of (5.6), (5.7) and (5.8). Then (5.5) follows.

c) Let us consider the case of $\left\langle\mathcal{P}_{2}\right\rangle=\left(\tau_{1},\left(\tau_{2}, \lambda^{\prime}\right)\right)$ such that the variable $y_{1}$ is attributed to the second arrival at the green vertex $\hat{v}$ of $\mathcal{G}^{(c)}$. The case when it is attributed to the red edge-box is similar and we do not discuss it here.

Let us denote by $q^{\prime}$ and $p^{\prime}$ the number of red and green vertices that lie to the left of $\hat{v}$ and by $q^{\prime \prime}$ and $p^{\prime \prime}-1$ the number of red and green vertices to the right of $\hat{v}$. We construct the skeleton $K$ and the tree $\mathcal{T}$ as it is described above. Then we perform the run of the sub-walk $\mathcal{W}=\mathcal{W}_{\left[0, t_{2}-1\right]}^{(\grave{\mathcal{T}})}, t_{2}=\xi_{\tau_{2}}$ following the prescriptions of $\left\langle\breve{v} \uplus \mathcal{G}^{(c)}\right\rangle_{s}^{(b)}$ and the rule $\Upsilon$. The vertex $\alpha=\hat{\mathcal{W}}\left(t_{2}-1\right)$ being determined, the exit cluster $\Delta(\alpha)=\left\{\gamma_{1}, \ldots, \gamma_{m}\right\}$ is also uniquely determined.

At the instant of time $\grave{\xi}_{\tau_{2}}$ the walk has to choose a vertex $\gamma^{\prime}$ from $\Delta(\alpha)$ such that $\gamma^{\prime}$ is situated on the distance of $\langle\Lambda\rangle=\lambda^{\prime}$ non-marked steps from $\breve{\beta}$. Therefore the indicator function of (5.12) $I_{\grave{\mathcal{W}}}\left(\lambda^{\prime}\right)$ is replaced by $I_{\dot{\mathcal{W}}}^{\left(\gamma_{1}, \ldots, \gamma_{m}\right)}\left(\lambda^{\prime}\right)$ that is non-zero only in the case when $\lambda^{\prime}$ takes one of the values that correspond to the length of one of the paths of non-marked edges from $\gamma_{i}$ to $\breve{\beta}$, if such paths exist. Thus,

$$
\sum_{\lambda^{\prime}} I_{\dot{\mathcal{W}}}^{\left(\gamma_{1}, \ldots, \gamma_{m}\right)}\left(\lambda^{\prime}\right) \leq m=|\Delta(\alpha)| \leq D
$$

Denoting $\mathbb{W}_{2 s}^{*}=\mathbb{W}_{2 s}\left(d_{1}, d_{2} ;\left\langle\breve{v} \uplus \mathcal{G}^{(c)}\right\rangle_{s},\langle\mathcal{H}\rangle, \Upsilon\right)$, we can write the following equality

$$
\mathbb{W}_{2 s}^{*}=\sqcup_{\grave{\mathcal{T}}}\left\{\mathcal{W}_{\left[0, \xi_{\tau_{2}-1}\right]}\right\} \otimes\left\{\left\langle\mathcal{W}\left(\xi_{\tau_{2}}\right)\right\rangle_{\lambda^{\prime}}\right\} \otimes \sqcup_{\mathcal{T}}\left\{\mathcal{W}_{\left[\xi_{\tau_{2}}+1,2 s\right]}^{(\mathcal{\dot { T }})}\right\}
$$

where the curly brackets denote the families of realizations of corresponding sub-walks and $\left\langle\mathcal{W}\left(\xi_{\tau_{2}}\right)\right\rangle_{\lambda^{\prime}}$ denotes the set of possible values $\gamma^{\prime}$. Then

$$
\sum_{\lambda^{\prime}}\left|\mathbb{W}_{2 s}^{*}\right| \leq \prod_{\mathcal{T}} \#\left\{\mathcal{W}_{\left[0, \xi_{\tau_{2}-1}\right]}\right\} \times \sum_{\lambda^{\prime}} \#\left\{\left\langle\mathcal{W}\left(\xi_{\tau_{2}}\right)\right\rangle_{l^{\prime}}\right\} \times e^{-\eta d_{2}} 2^{q^{\prime \prime}} D^{p^{\prime \prime}-1} \#\{\dot{\mathcal{T}}\}
$$

where we have used inequality (5.13).

It follows from (5.14) that

$$
\sum_{\lambda^{\prime}} \#\left\{\left\langle\mathcal{W}\left(\xi_{\tau_{2}}\right)\right\rangle_{l^{\prime}}\right\} \leq D .
$$

Using this inequality and estimates

$$
\#\left\{\mathcal{W}_{\left[0, \grave{\xi}_{\tau_{2}-1}\right]}\right\} \leq 2^{q^{\prime}} D^{p^{\prime}}
$$


and

$$
\prod_{\dot{\mathcal{T}}} 1 \cdot \#\{\dot{\mathcal{T}}\} \leq e^{-\eta d_{1}} \mathrm{t}_{s}
$$

we conclude that

$$
\sum_{\lambda^{\prime}}\left|\mathbb{W}_{2 s}^{*}\right| \leq 2^{|\bar{q}|} D^{|\bar{p}|} e^{-\eta\left(d_{1}+d_{2}\right)} \mathrm{t}_{s} .
$$

Remembering (3.23), it is easy to show that (5.6) is true in the case under consideration.

\subsubsection{The cases of $N=2, R \geq 3$}

Let us consider $\mathcal{P}_{3}$ such that the first two cells at $\breve{\beta}$ are given by the instants $\left\langle\left(x_{1}, x_{2}\right)\right\rangle=\left(\tau_{1}, \tau_{2}\right)$ while the third one is represented by the mirror cell. The presence of this mirror, $m_{2}=1$ means that in the Dyck-type part of the walk, and in the corresponding tree the vertices $\check{v}_{1}=\mathfrak{R}\left(\xi_{\tau_{1}}\right)$ and $\check{v}_{2}=\mathfrak{R}\left(\xi_{\tau_{2}}\right)$ lie on the same branch of edges that starts at the root vertex $\mathfrak{b}_{0}$. Therefore the tree $\mathcal{T}$ is of the following structure: we choose a length $l_{1}$ and construct the branch $\mathcal{B}_{1}$ of $l_{1}$ edges that starts by $\mathfrak{b}_{0}$ and ends by $\check{v}_{1}=\mathfrak{b}_{l_{1}}$. Then we attach to $\check{v}_{1}$ another linear branch $\mathcal{B}_{2}$ of $l_{2}$ edges that ends by $\check{v}_{2}$. We attach the exit sub-cluster $\check{\Delta}_{1}$ to $v_{1}$ at the instant $l_{1}$ of the chronological run $\mathfrak{R}\left(\mathcal{B}_{1} \uplus \mathcal{B}_{2}\right)$ and the sub-cluster $\check{\Delta}_{3}$ to the vertex $v_{1}$ at the instant $l_{1}+2 l_{2}+1$ of $\mathfrak{R}\left(\mathcal{B}_{1} \uplus \mathcal{B}_{2}\right)$.

Then we attach the sub-cluster $\check{\Delta}_{2}$ at the vertex $\check{v}_{2}$. Regarding $2\left(l_{1}+l_{2}\right)+1+d_{1}+d_{2}+d_{3}$ vertices of the obtained skeleton, we attach to them the sub-trees of the total number of edges $s-\left(l_{1}+l_{2}+d_{1}+d+2+d_{3}\right)$. Using three times inequalities of the from (5.7) we easily get exponential estimates of (5.6) in this case.

To complete the study of the initial step of the proof of Lemma 5.1, we consider the case of numerous imported cells of the form $\mathcal{P}_{R}=\left(z_{1},\left(y_{1}, \Lambda, \psi_{1}, \ldots, \psi_{f}\right)\right)$, where $f=f_{1}^{\prime \prime}$ and $R=3+f$. We assume for simplicity that $\left\langle\left(z_{1}, y_{1}\right)\right\rangle=\left(\tau_{1}, \tau_{2}\right)$ and that $\tau_{1}<\tau_{2}$. The reasoning presented below can be applied without any changes to the case of imported cells generated by the local BTS instants $\left\langle\left(z_{1}, z_{2}\right)\right\rangle=\left(\tau_{1}, \tau_{2}\right)$. Let us point out that in this situation either $f=0$ or $f=1$ (see inequality (3.22)). However, we include into considerations the general case of greater values of $f$. Another remark is that we can ignore the presence of the proper cell $\left\langle z_{1}\right\rangle=\tau_{1}$ with the exit sub-cluster $\breve{\Delta}_{1}$ at $\breve{\beta}$ and consider the imported cells and corresponding exit sub-clusters only. We also assume for simplicity that $y_{2}$ is attributed to a blue $r$-vertex $\hat{v}$ of $\mathcal{G}^{(c)}$.

To get a realization of $\langle\mathcal{H}\rangle$, we take an integer $f$ and then attribute numerical values to the variables $\Lambda, \psi_{1}, \ldots, \psi_{f}$ given by $\lambda^{\prime}, \psi_{1}^{\prime}, \ldots, \psi_{f}^{\prime}$. Let us take a tree $\grave{\mathcal{T}}=\grave{\mathcal{T}}_{\tau_{2}}$ and consider a part of the chronological run over it $\Re_{\left[0, t^{\prime}-1\right]}$ with $t^{\prime}=\xi_{\tau_{2}}$. Following this run, we construct a sub-walk $\mathcal{W}_{\left[0, t^{\prime}-1\right]}$ according to the rules prescribed by $\left\langle\breve{v} \uplus \mathcal{G}^{(c)}\right\rangle_{s}^{(b)}$ and $\Upsilon$. At the instant of time $t^{\prime}$, the walk has to join a vertex $\gamma$ of $g\left(\mathcal{W}_{\left[0, t^{\prime}-1\right]}\right)$ prescribed by the values of marked instants of the edge-boxes attached to $\hat{v}$. This vertex $\gamma$ is uniquely determined and therefore we are able to conclude whether the set of numerical data $f,\left(\lambda^{\prime}, \psi_{1}^{\prime}, \ldots, \psi_{f}^{\prime}\right)$ is compatible with $\mathcal{W}_{\left[0, t^{\prime}-1\right]}$ or not. We mean that it becomes clear whether there exists a path from $\gamma$ to $\breve{\beta}$ of $\lambda^{\prime}$ non-marked steps that the walk can perform according to the rules $\Upsilon$ or not. The same concern $f$ consecutive returns to $\breve{\beta}$ with the help of $\psi_{l}^{\prime}$ non-marked steps.

The $f+1$ nest cells are uniquely determined in $\grave{\mathcal{T}}_{\tau_{2}}$ and the exit sub-clusters of the total cardinality $\check{D}=D-(f+1)$ are to be distributed to these nest cells. Let us denote by $\bar{d}_{f+1}$ 
this distribution. We also denote by $\mathbb{T}_{s}\left(\grave{\mathcal{T}}_{\tau_{2}} \uplus\left\{\check{\Delta}_{1}, \ldots, \check{\Delta}_{f+1}\right\}\right)$ a collection of Catalan trees constructed over the base tree $\grave{\mathcal{T}}$ with the exit sub-clusters $\check{\Delta}_{j}$ attached.

Using (5.8) and (5.11) several times, one can easily prove the exponential estimate for the number of trees

$$
\left|\mathbb{T}_{s}\left(\grave{\mathcal{T}}_{\tau_{2}} \uplus\left\{\check{\Delta}_{1}, \ldots, \check{\Delta}_{f+1}\right\}\right)\right| \leq e^{-\eta \check{D}}\left|\mathbb{T}_{s}\left(\mathcal{T}_{\tau_{2}}\right)\right| .
$$

By changing somehow the point of view, we can say that given $\left\langle\breve{v} \uplus \mathcal{G}^{(c)}\right\rangle_{s}^{(b)}$ and $\Upsilon$, the set of all possible values of $f$ and $\left\langle\left(\Lambda, \psi_{1}, \ldots, \psi_{f}\right)\right\rangle$ is filtered by the run of the walk $\mathcal{W}_{\left[0, t^{\prime}-1\right]}$. The values $f$ and $\check{D}=D-(f+1)$ depend on the realization of $\mathcal{W}_{\left[0, t^{\prime}-1\right]}$. With the help of the filtration principle, we get the following inequality,

$$
\begin{gathered}
\left|\sqcup_{\langle\mathcal{H}\rangle} \mathbb{W}_{2 s}^{(\grave{\mathcal{T}})}\left(D,\left\langle\breve{v} \uplus \mathcal{G}^{(c)}\right\rangle_{s}^{(b)},\langle\mathcal{H}\rangle, \Upsilon\right)\right| \\
\leq 2^{|\bar{q}|} D^{|\bar{p}|} \sup _{f}\left\{e^{\eta(f+1)}\left(\begin{array}{c}
\check{D}+f \\
f
\end{array}\right)\right\} e^{-\eta D} \sum_{\grave{\mathcal{T}}}\left|\mathbb{T}_{s}\left(\mathcal{T}_{\tau_{2}}\right)\right|,
\end{gathered}
$$

where the superscript $\mathcal{T}$ means that the walks have this tree as the first part of the underlying trees. Taking into account the upper bound $f \leq K=1$ (see (3.22)), we can apply to the right-hand side of (5.16) relations (3.21) and (3.23) and write that

$$
\sup _{f}\left\{e^{\eta(f+1)}\left(\begin{array}{c}
\check{D}+f \\
f
\end{array}\right)\right\} \leq e^{2 \eta} h_{0}^{2} e^{e D / h_{0}} .
$$

Repeating the reasoning of (5.8), we get from (5.16) and (5.17) the upper bound (5.6).

\subsubsection{The general step of recurrence}

The general step of recurrent estimate of (5.6) is to show that if this estimate is true for $N=I+J+K$, then it is true in the case of $N^{\prime}=N+1$, where $N^{\prime}=I^{\prime}+J^{\prime}+K^{\prime}$. Let us consider the case when $K^{\prime}=K+1$ and $I^{\prime}=I, J^{\prime}=J$. This means that if the set $\left(\bar{x}_{I}, \bar{y}_{J}, \bar{z}_{k}\right)$ is represented by $N$ marked instants of time $\tau_{1}<\tau_{2}<\cdots<\tau_{N}$, then $\tau_{N+1}>\tau_{n}$ and $z_{K+1}=\tau_{N+1}$. Obviously, the numbers $f_{K+1}^{\prime \prime}=f$ and $\bar{\varphi}^{(K+1)}=\left(\varphi_{1}^{(K+1)}, \ldots, \varphi_{f}^{(K+1)}\right)$ are also joined to the set of parameters $\left\langle\mathcal{P}_{R}\right\rangle(3.17)$.

Let us briefly describe the steps that we perform to get the estimate needed. Regarding the vertices and the edge-boxes of realization of the color diagram $\left\langle\mathcal{G}^{(c)}(\bar{p}, \bar{q}, \bar{\nu})\right\rangle_{s}$, we separate the edge-boxes of each vertex into two groups in dependence of whether the values in the boxes are less than $\tau_{N+1}$ or greater than $\tau_{N+1}$. Clearly, the vertex attached by the edge-box with $\tau_{N+1}$ plays a special role here. By this procedure, we obtain realizations of two sub-diagrams $\langle\grave{\mathcal{G}}\rangle$ and $\langle\mathcal{G}\rangle$ determined in obvious way.

The underlying trees $\mathcal{T}_{s}=\mathcal{T}\left(\mathcal{W}_{2 s}\right)$ of the walks are to be of the following structure: there exists a branch $\mathcal{B}_{N+1}$ such that the descending path from the extreme vertex $\check{u}_{N+1}$ to the root $\mathfrak{b}_{0}$ is of the total length not less than $\left|\bar{\phi}^{(K+1)}\right|=\sum_{i=1}^{f} \varphi_{i}^{(K+1)}$. At the vertex $\check{v}_{N+1}$ and corresponding $f$ vertices of the descending part of $\mathcal{B}_{N+1}$, the sub-clusters of the total number of $D_{N+1}$ edges are attached. Then the remaining edges are used to construct sub-trees attached to $l_{N+1}+D_{N+1}-f$ vertices. We denote this part of $\mathcal{T}$ by $\mathcal{T}$.

It is clear that the set of the walks under consideration can be represented in the form of the right-hand side of (5.12) with $\tau_{2}$ replaced by $\tau_{N+1}$ and that the exponential estimate with 
the factor $e^{-\eta D_{N+1}}$ can be obtained for the family of trees $\{\dot{\mathcal{T}}\}$ (see also inequality (5.13), where $q^{\prime \prime}$ and $p^{\prime \prime}$ are determined with the help of sub-diagram $\mathcal{G}$ ). Using (5.17), it is not hard to complete the proof of (5.6) in the case of $N^{\prime}=N+1$. We omit the detailed computations here because they repeat in major part those performed earlier in this sub-section (see also [12] for more discussion of the general step of recurrent estimates).

Acknowledgements. The author is grateful to V. Vengerovsky for the careful reading of the manuscript and useful remarks.

\section{References}

[1] G. W. Anderson, A. Guionnet and O. Zeitouni, An introduction to random matrices, Cambridge Studies in Advanced Mathematics 118 , Cambridge University Press, Cambridge (2010) $492 \mathrm{pp}$.

[2] Z. D. Bai and Y. Q. Yin, Necessary and sufficient conditions for the almost sure convergence of the largest eigenvalue of Wigner matrices, Ann. Probab. 16 1729-1741 (1988)

[3] M. Bauer and O. Golinelli, Random incidence matrices: moments of the spectral density, J. Statist. Phys. 103 (2001) 301-337

[4] B. Bollobás, Random Graphs, Cambridge studies in advances mathematics 73, Cambridge University Press, Cambridge (2001) 498 pp.

[5] L. Erdős, A. Knowles, H.-T. Yau, J. Yin, Spectral statistics of Erdős-Rényi graphs I: Local semicircle law, Ann. Probab. 41 (2013) 2279-2375

[6] O. N. Feldheim and S. Sodin, A universality result for the smallest eigenvalues of certain sample covariance matrices, Geom. Funct. Anal. 20 (2010) 88-123

[7] Z. Füredi and J. Komlós, The eigenvalues of random symmetric matrices, Combinatorica, 1 (1981) 233-241

[8] S. Geman, A limit theorem for the norm of random matrices, Ann. Probab. 8 (1980) $252-261$

[9] A. Khorunzhy, Sparse random matrices: spectral edge and statistics of rooted trees, Adv. Appl. Probab. 33 (2001) 124-140

[10] O. Khorunzhiy, High moments of large Wigner random matrices and asymptotic properties of the spectral norm, Random Oper. Stoch. Equ. 20 (2012) 25-68

[11] O. Khorunzhiy, On correlation function of high moments of Wigner random matrices, Random Oper. Stoch. Equations 21 (2013) 345-374

[12] O. Khorunzhiy, On high moments and the spectral norm of large dilute Wigner random matrices, J. Math. Phys., Analysis, Geom. 10 (2014) 64-125

[13] A. Khorunzhy, B. Khoruzhenko, L. Pastur, and M. Shcherbina, The large-n limit in statistical mechanics and the spectral theory of disordered systems, in: Phase Transitions and Critical Phenomena Vol. 15, pp. 74-239, Academic Press, London, 1992 
[14] O. Khorunzhiy, W. Kirsch, and P. Müller, Lifshitz tails for spectra of Erdős-Rényi random graphs, Ann. Appl. Probab. 16 (2006) 295-309

[15] O. Khorunzhy, M. Shcherbina, and V. Vengerovsky, Eigenvalue distribution of large weighted random graphs, J. Math. Phys. 45 (2004) 1648-1672

[16] O. Khorunzhiy and V. Vengerovsky, Even walks and estimates of high moments of large Wigner random matrices, Preprint arXiv:0806.0157

[17] M. Krivelevich and B. Sudakov, The largest eigenvalue of sparse random graphs, :it Combin. Probab. Comput. 12 (2003) 61-72

[18] M.L. Mehta, Random Matrices, Amsterdam: Elsevier/Academic Press (2004) 706 pp.

[19] A.D. Mirlin and Ya.V. Fyodorov, Universality of level correlation function of sparse random matrices, J. Phys. A 24 (1991) 2273-2286

[20] C. Porter (ed.), Statistical Theories of Spectra: Fluctuations (1965) Acad. Press, NewYork

[21] G.J. Rodgers and A.J. Bray, Density of states of a sparse random matrix, Phys. Rev. B 37 (1988) 3557-3562

[22] Ya. Sinai and A. Soshnikov, Central limit theorem for traces of large symmetric matrices with independent matrix elements, Bol. Soc. Brazil. Mat. 29 (1998) 1-24

[23] Ya. Sinai and A. Soshnikov, A refinement of Wigner's semicircle law in a neighborhood of the spectrum edge for random symmetric matrices, Func. Anal. Appl. 32 (1998) 114-131

[24] S. Sodin, The Tracy-Widom law for some sparse random matrices, Journal of Statistical Physics 136 (2009) 834-841

[25] A. Soshnikov, Universality at the edge of the spectrum in Wigner random matrices, Commun. Math. Phys. 207 (1999) 697-733

[26] A. Soshnikov, A note on universality of the distribution of the largest eigenvalues in certain sample covariance matrices, J. Stat. Physics 108(2002 1033-1056

[27] C. A. Tracy and H. Widom, Level spacing distribution and the Airy kernel, Commun. Math. Phys. 159 (1994) 151-174

[28] E. Wigner, Characteristic vectors of bordered matrices with infinite dimensions, Ann. Math. 62 (1955) 548-564 